Network Working Group

Request for Comments: 5000

RFC Editor

STD : 1

Obsoletes: 3700

Category: Informational

$\mathrm{USC} / \mathrm{ISI}$

May 2008

\title{
Internet Official Protocol Standards
}

Status of This Memo

This memo provides information for the Internet community. It does not specify an Internet standard of any kind. Distribution of this memo is unlimited.

\section{IESG Note}

This document obsoletes RFC 3700. It also reclassifies RFCs that were previously published as STD 1 as Historic. More specifically, this RFC moves RFCs 3000, 3300, 3600, and 3700 to Historic status.

Abstract

This document is published by the RFC Editor to provide a summary of the current standards protocols (as of 18 February 2008). It lists those official protocol standards, Best Current Practice, and

Experimental RFCs that have not been obsoleted; it is not a complete index to the RFC series. Newly published RFCs and RFCs whose status has changed are starred.

For an up-to-date list, see http://www.rfc-editor.org/rfcxx00.html, which is updated daily.

Table of Contents

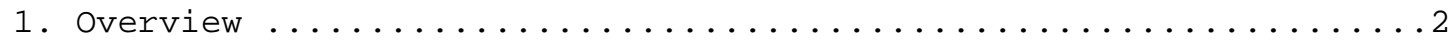

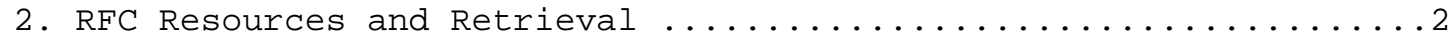

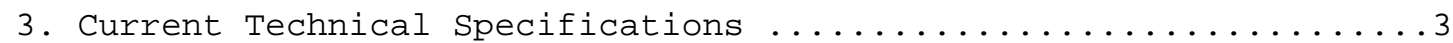

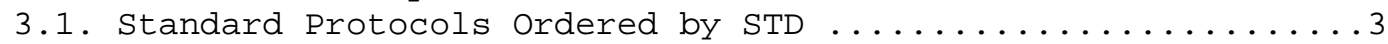

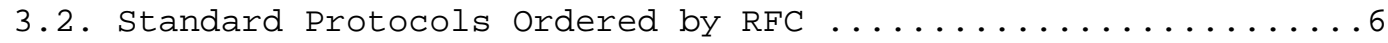

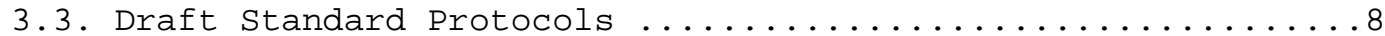

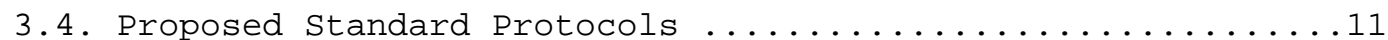

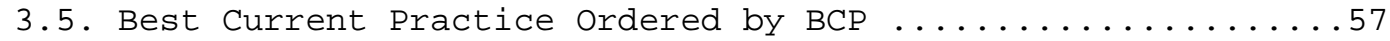

3.6. Best Current Practice Ordered by RFC ..............62

3.7. Experimental Protocols .......................67

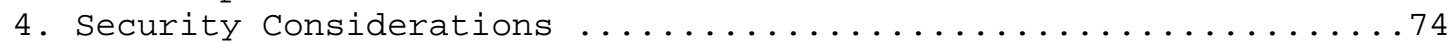




\section{Overview}

This memo contains a snapshot of the state of standardization of protocols used in the Internet, as determined by the Internet Engineering Task Force (IETF). It is a 18 February 2008 snapshot of the current official protocol standards list and the Best Current Practice list, which is updated daily and is available from the RFC Editor Web site.

The RFC Editor publishes Request for Comments (RFC) documents that are the output of 4 streams: IAB, IETF, IRTF, and Independent Submission via the RFC Editor.

This memo is published by the RFC Editor for the IESG and IAB, in which the categorizations are in accordance with section 2.1 of "The Internet Standards Process -- Revision 3", RFC 2026, which specifies the rules and procedures by which all Internet standards are set. Sections 3.1 - 3.7 of this memo contain the lists of protocols in each stage of standardization - Standard, Draft Standard, Proposed Standard, Experimental, as well as Best Current Practice documents. Protocols that are new to this document or have been moved from one protocol level to another, or that differ from the previous edition of this document are marked with an asterisk. Informational and Historic RFCs are not included. This list is not a complete index to RFCs.

2. RFC Resources and Retrieval

This list is a live document that is updated daily on the RFC Editor site at:

http://www.rfc-editor.org/rfcxx00.html

RFCs are available online via http and ftp as:

http: //www.rfc-editor.org/rfc/rfcXXXX.txt

ftp://ftp.rfc-editor.org/in-notes/rfcXXXX.txt

where XXXX represents the corresponding RFC number.

The RFC Editor maintains the official repository of all RFCs and indexes to them. The site also provides additional information about the history of the RFC Editor, current submission and review procedures, and editorial and publication procedures. Please see the RFC Editor site for all other $\mathrm{RFC}$ related information:

http://www.rfc-editor.org 


\section{Current Technical Specifications}

3.1. Standard Protocols Ordered by STD

[Note: an asterisk at the end of a line indicates a change from the previous edition of this document.]

\begin{tabular}{|c|c|c|c|}
\hline Mnemonic & Title & $\mathrm{RFC \#}$ & TD\# \\
\hline-------- & Internet Official Protocol Standards & $5000 *$ & 1 \\
\hline-------- & $\begin{array}{l}\text { [Reserved for Assigned Numbers. See RFC } 1700 \text { and } \\
\text { RFC 3232.] }\end{array}$ & $\mathrm{xxx}$ & 2 \\
\hline-------- & $\begin{array}{l}\text { Requirements for Internet Hosts - Communication } \\
\text { Layers }\end{array}$ & 1122 & 3 \\
\hline-------- & $\begin{array}{l}\text { Requirements for Internet Hosts - Application and } \\
\text { Support }\end{array}$ & 1123 & 3 \\
\hline-------- & [Reserved for Router Requirements. See RFC 1812.] & $\mathrm{x} x \mathrm{x}$ & 4 \\
\hline IP & Internet Protocol & 791 & 5 \\
\hline ICMP & Internet Control Message Protocol & 792 & 5 \\
\hline-------- & Broadcasting Internet Datagrams & 919 & 5 \\
\hline------ & $\begin{array}{l}\text { Broadcasting Internet datagrams in the presence of } \\
\text { subnets }\end{array}$ & 922 & 5 \\
\hline-------- & Internet Standard Subnetting Procedure & 950 & 5 \\
\hline IGMP & Host extensions for IP multicasting & 1112 & 5 \\
\hline UDP & User Datagram Protocol & 768 & 6 \\
\hline $\mathrm{TCP}$ & Transmission Control Protocol & 793 & 7 \\
\hline TELNET & Telnet Protocol Specification & 854 & 8 \\
\hline FTP & File Transfer Protocol & 959 & 9 \\
\hline---- & $\begin{array}{l}\text { ["Mail routing and the domain system". Was RFC } 974 \\
\text { (STANDARD), Now HISTORIC.] }\end{array}$ & $\mathrm{xxx}$ & 10 \\
\hline SMTP & $\begin{array}{l}\text { ["Simple Mail Transfer Protocol". Was RFC } 821 \\
\text { (STANDARD), Obsoleted by RFC } 2821 \text { (PROPOSED } \\
\text { STANDARD)] }\end{array}$ & $\mathrm{xxx}$ & 10 \\
\hline------ & $\begin{array}{l}\text { ["SMTP Service Extensions". Was RFC } 1869 \\
\text { (STANDARD), Obsoleted by RFC } 2821 \text { (PROPOSED } \\
\text { STANDARD)] }\end{array}$ & $\mathrm{xxx}$ & 10 \\
\hline SMTP-SIZE & SMTP Service Extension for Message Size Declaration & 1870 & 10 \\
\hline MAIL & $\begin{array}{l}\text { ["STANDARD FOR THE FORMAT OF ARPA INTERNET TEXT } \\
\text { MESSAGES". Was RFC } 822 \text { (STANDARD), Obsoleted by } \\
\text { RFC } 2822 \text { (PROPOSED STANDARD)] }\end{array}$ & $\mathrm{xxx}$ & 11 \\
\hline-------- & $\begin{array}{l}\text { [Reserved for Network Time Protocol (NTP). See RFC } \\
\text { 1305.] }\end{array}$ & $\mathrm{xxx}$ & 12 \\
\hline DOMAIN & Domain names - concepts and facilities & 1034 & 13 \\
\hline DOMAIN & Domain names - implementation and specification & 1035 & 13 \\
\hline-------- & $\begin{array}{l}\text { [Was Mail Routing and the Domain System. Now } \\
\text { Historic.] }\end{array}$ & $\mathrm{xxx}$ & 14 \\
\hline-------- & [Was Simple Network Management Protocol. Now & $\mathrm{xxx}$ & 15 \\
\hline
\end{tabular}




\begin{tabular}{|c|c|c|}
\hline SMI & $\begin{array}{l}\text { Structure and identification of management } \\
\text { information for TCP/IP-based internets }\end{array}$ & 1155 \\
\hline Concise-MI & Concise MIB definitions & 1212 \\
\hline MIB-II & $\begin{array}{l}\text { Management Information Base for Network Management } \\
\text { of TCP/IP-based internets:MIB-II }\end{array}$ & 1213 \\
\hline-------- & $\begin{array}{l}\text { [Was Exterior Gateway Protocol (RFC 904). Now } \\
\text { Historic.] }\end{array}$ & $\mathrm{xxx}$ \\
\hline NETBIOS & $\begin{array}{l}\text { Protocol standard for a NetBIOS service on a } \\
\text { TCP/UDP transport: Concepts and methods }\end{array}$ & 1001 \\
\hline NETBIOS & $\begin{array}{l}\text { Protocol standard for a NetBIOS service on a } \\
\text { TCP/UDP transport: Detailed specifications }\end{array}$ & 1002 \\
\hline $\mathrm{ECHO}$ & Echo Protocol & 862 \\
\hline DISCARD & Discard Protocol & 863 \\
\hline CHARGEN & Character Generator Protocol & 864 \\
\hline QUOTE & Quote of the Day Protocol & 865 \\
\hline USERS & Active users & 866 \\
\hline DAYTIME & Daytime Protocol & 867 \\
\hline TIME & Time Protocol & 868 \\
\hline TOPT-BIN & Telnet Binary Transmission & 0 \\
\hline TOPT-ECHO & Telnet Echo Option & 0 \\
\hline TOPT-SUPP & Telnet Suppress Go Ahead Option & 0 \\
\hline-------- & Telnet Status Option & 859 \\
\hline TOPT-TIM & Telnet Timing Mark Option & 0 \\
\hline TOPT-EXTOP & Telnet Extended Options: List Option & 0 \\
\hline TFTP & The TFTP Protocol (Revision 2) & 1350 \\
\hline-------- & $\begin{array}{l}\text { [Was Routing Information Protocol (RIP). Replaced } \\
\text { by STD 56.] }\end{array}$ & $\mathrm{xxx}$ \\
\hline $\mathrm{TP}-\mathrm{TCP}$ & ISO Transport Service on top of the TCP Version: 3 & 1006 \\
\hline IP-FDDI & Transmission of IP and ARP over FDDI Networks & 1390 \\
\hline ARP & $\begin{array}{l}\text { Ethernet Address Resolution Protocol: Or } \\
\text { Converting Network Protocol Addresses to } 48 . \text { bit } \\
\text { Ethernet Address for Transmission on Ethernet } \\
\text { Hardware }\end{array}$ & 826 \\
\hline RARP & A Reverse Address Resolution Protocol & 903 \\
\hline IP-ARPA & $\begin{array}{l}\text { [Was BBN Report } 1822 \text { (IMP/Host Interface). Now } \\
\text { Historic.] }\end{array}$ & $\mathrm{xxx}$ \\
\hline IP $-W B$ & Host Access Protocol specification & 907 \\
\hline$I P-E$ & $\begin{array}{l}\text { A Standard for the Transmission of IP Datagrams } \\
\text { over Ethernet Networks }\end{array}$ & 894 \\
\hline IP-EE & $\begin{array}{l}\text { Standard for the transmission of IP datagrams over } \\
\text { experimental Ethernet networks }\end{array}$ & 895 \\
\hline IP-IEEE & $\begin{array}{l}\text { Standard for the transmission of IP datagrams over } \\
\text { IEEE } 802 \text { networks }\end{array}$ & 1042 \\
\hline IP-DC & DCN Local-Network Protocols & 891 \\
\hline $\mathrm{IP}-\mathrm{HC}$ & $\begin{array}{l}\text { Internet Protocol on Network System's } \\
\text { HYPERchannel: Protocol Specification }\end{array}$ & 1044 \\
\hline IP $-\mathrm{ARC}$ & Transmitting IP traffic over ARCNET networks & 1201 \\
\hline
\end{tabular}




\begin{tabular}{|c|c|c|}
\hline IP-SLIP & $\begin{array}{l}\text { Nonstandard for transmission of IP datagrams over } \\
\text { serial lines: SLIP }\end{array}$ & 1055 \\
\hline IP-NETBIOS & $\begin{array}{l}\text { Standard for the transmission of IP datagrams over } \\
\text { NetBIOS networks }\end{array}$ & 1088 \\
\hline IP-IPX & $\begin{array}{l}\text { Standard for the transmission of } 802.2 \text { packets } \\
\text { over IPX networks }\end{array}$ & 1132 \\
\hline---- & $\begin{array}{l}\text { [Reserved for Definitions of Managed Objects for } \\
\text { the Ethernet-like Interface Types. See RFC 3638.] }\end{array}$ & $\mathrm{x} x \mathrm{x}$ \\
\hline PPP & The Point-to-Point Protocol (PPP) & 1661 \\
\hline $\mathrm{PP}-\mathrm{HDLC}$ & $\mathrm{PPP}$ in HDLC-like Framing & 1662 \\
\hline$P-\operatorname{SMDS}$ & $\begin{array}{l}\text { The Transmission of IP Datagrams over the SMDS } \\
\text { Service }\end{array}$ & 1209 \\
\hline oP 3 & Post Office Protocol - Version 3 & 1939 \\
\hline OSPF 2 & OSPF Version 2 & 2328 \\
\hline IP-FR & Multiprotocol Interconnect over Frame Relay & 2427 \\
\hline IP 2 & RIP Version 2 & 2453 \\
\hline IP $2-A P P$ & RIP Version 2 Protocol Applicability statement & 1722 \\
\hline MIv2 & $\begin{array}{l}\text { Structure of Management Information Version } 2 \\
\text { (SMIV2) }\end{array}$ & 2578 \\
\hline CONV-MIB & Textual Conventions for SMIv2 & 2579 \\
\hline $\mathrm{ONF}-\mathrm{MIB}$ & Conformance statements for SMIv2 & 2580 \\
\hline MON-MIB & $\begin{array}{l}\text { Remote Network Monitoring Management Information } \\
\text { Base }\end{array}$ & 2819 \\
\hline SMTP-Pipe & SMTP Service Extension for Command Pipelining & 2920 \\
\hline ONE-PASS & A One-Time Password System & 2289 \\
\hline $\mathrm{RCH}-\mathrm{SNMP}$ & $\begin{array}{l}\text { An Architecture for Describing Simple Network } \\
\text { Management Protocol (SNMP) Management Frameworks }\end{array}$ & 3411 \\
\hline PD-SNMP & $\begin{array}{l}\text { Message Processing and Dispatching for the Simple } \\
\text { Network Management Protocol (SNMP) }\end{array}$ & 3412 \\
\hline SNMP - APP & $\begin{array}{l}\text { Simple Network Management Protocol (SNMP) } \\
\text { Applications }\end{array}$ & 3413 \\
\hline USM-SNMPV3 & $\begin{array}{l}\text { User-based Security Model (USM) for version } 3 \text { of } \\
\text { the Simple Network Management Protocol (SNMPv3) }\end{array}$ & 3414 \\
\hline VACM-SNMP & $\begin{array}{l}\text { View-based Access Control Model (VACM) for the } \\
\text { Simple Network Management Protocol (SNMP) }\end{array}$ & 3415 \\
\hline PS-MIB & $\begin{array}{l}\text { Version } 2 \text { of the Protocol Operations for the } \\
\text { Simple Network Management Protocol (SNMP) }\end{array}$ & 3416 \\
\hline TRANS-MIB & $\begin{array}{l}\text { Transport Mappings for the Simple Network } \\
\text { Management Protocol (SNMP) }\end{array}$ & 3417 \\
\hline SNMPV2-MIB & $\begin{array}{l}\text { Management Information Base (MIB) for the Simple } \\
\text { Network Management Protocol (SNMP) }\end{array}$ & 3418 \\
\hline $\mathrm{UTF}-8$ & UTF-8, a transformation format of ISO 10646 & 3629 \\
\hline RTP & $\begin{array}{l}\text { RTP : A Transport Protocol for Real-Time } \\
\text { Applications }\end{array}$ & 3550 \\
\hline $\mathrm{RTP}-\mathrm{AV}$ & $\begin{array}{l}\text { RTP Profile for Audio and Video Conferences with } \\
\text { Minimal Control }\end{array}$ & 3551 \\
\hline
\end{tabular}


XDR XDR: External Data Representation Standard $\quad 4506 * \quad 67$

ABNF Augmented BNF for Syntax Specifications: ABNF 5234 * 68

3.2. Standard Protocols Ordered by RFC

[Note: an asterisk at the end of a line indicates a change from the previous edition of this document.]

\begin{tabular}{|c|c|c|c|}
\hline Mnemonic & Title & STD\# & \\
\hline ABNE & Augmented BNF for Syntax Specifications: ABNF & 68 & \\
\hline-------- & Internet Official Protocol Standards & 1 & $5000 x$ \\
\hline $\mathrm{XDR}$ & XDR: External Data Representation Standard & 67 & $4506^{*}$ \\
\hline------- & Uniform Resource Identifier (URI): Generic Syntax & 66 & 3986 \\
\hline $\mathrm{UTF}-8$ & UTF-8, a transformation format of ISO 10646 & 63 & 3629 \\
\hline $\mathrm{RTP}-\mathrm{AV}$ & $\begin{array}{l}\text { RTP Profile for Audio and Video Conferences with } \\
\text { Minimal Control }\end{array}$ & 65 & 3551 \\
\hline $\mathrm{RTP}$ & $\begin{array}{l}\text { RTP : A Transport Protocol for Real-Time } \\
\text { Applications }\end{array}$ & 64 & 3550 \\
\hline SNMP v2-MIB & $\begin{array}{l}\text { Management Information Base (MIB) for the Simple } \\
\text { Network Management Protocol (SNMP) }\end{array}$ & 62 & 3418 \\
\hline TRANS-MIB & $\begin{array}{l}\text { Transport Mappings for the Simple Network } \\
\text { Management Protocol (SNMP) }\end{array}$ & 62 & 3417 \\
\hline OPS-MIB & $\begin{array}{l}\text { Version } 2 \text { of the Protocol Operations for the } \\
\text { Simple Network Management Protocol (SNMP) }\end{array}$ & 62 & 3416 \\
\hline VACM-SNMP & $\begin{array}{l}\text { View-based Access Control Model (VACM) for the } \\
\text { Simple Network Management Protocol (SNMP) }\end{array}$ & 62 & 3415 \\
\hline USM-SNMPV3 & $\begin{array}{l}\text { User-based Security Model (USM) for version } 3 \text { of } \\
\text { the Simple Network Management Protocol (SNMPv3) }\end{array}$ & 62 & 3414 \\
\hline SNMP - APP & $\begin{array}{l}\text { Simple Network Management Protocol (SNMP) } \\
\text { Applications }\end{array}$ & 62 & 3413 \\
\hline MPD-SNMP & $\begin{array}{l}\text { Message Processing and Dispatching for the } \\
\text { Simple Network Management Protocol (SNMP) }\end{array}$ & 62 & 3412 \\
\hline ARCH-SNMP & $\begin{array}{l}\text { An Architecture for Describing Simple Network } \\
\text { Management Protocol (SNMP) Management Frameworks }\end{array}$ & 62 & 3411 \\
\hline SMTP-Pipe & SMTP Service Extension for Command Pipelining & 60 & 2920 \\
\hline RMON-MIB & $\begin{array}{l}\text { Remote Network Monitoring Management Information } \\
\text { Base }\end{array}$ & 59 & 2819 \\
\hline $\mathrm{CONF}-\mathrm{MIB}$ & Conformance Statements for SMIv2 & 58 & \\
\hline CONV-MIB & Textual Conventions for SMIv2 & 58 & 2579 \\
\hline SMIV2 & $\begin{array}{l}\text { Structure of Management Information Version } 2 \\
\quad(\text { SMIV } 2)\end{array}$ & 58 & 2578 \\
\hline RIP 2 & RIP Version 2 & 56 & \\
\hline IP-FR & Multiprotocol Interconnect over Frame Relay & 55 & 2427 \\
\hline OSPE2 & OSPF Version 2 & 54 & 2328 \\
\hline ONE-PASS & A One-Time Password System & 61 & 2289 \\
\hline POP 3 & Post Office Protocol - Version 3 & 53 & 1939 \\
\hline
\end{tabular}




\begin{tabular}{|c|c|c|c|}
\hline SMTP-SIZE & $\begin{array}{l}\text { SMTP Service Extension for Message Size } \\
\text { Declaration }\end{array}$ & 10 & 1870 \\
\hline RIP 2-APP & RIP Version 2 Protocol Applicability statement & 57 & 1722 \\
\hline PPP-HDLC & $\mathrm{PPP}$ in HDLC-like Framing & 51 & 1662 \\
\hline $\mathrm{PPP}$ & The Point-to-Point Protocol (PPP) & 51 & 1661 \\
\hline IP-FDDI & Transmission of IP and ARP over FDDI Networks & 36 & 1390 \\
\hline TETP & The TFTP Protocol (Revision 2) & 33 & 1350 \\
\hline MIB-II & $\begin{array}{l}\text { Management Information Base for Network } \\
\text { Management of TCP/IP-based internets:MIB-II }\end{array}$ & 17 & 121 \\
\hline Concise-MI & Concise MIB definitions & 16 & 1212 \\
\hline IP-SMDS & $\begin{array}{l}\text { The Transmission of IP Datagrams over the SMDS } \\
\text { Service }\end{array}$ & 52 & 1209 \\
\hline IP $-\mathrm{ARC}$ & Transmitting IP traffic over ARCNET networks & 46 & 1201 \\
\hline SMI & $\begin{array}{l}\text { Structure and identification of management } \\
\text { information for TCP/IP-based internets }\end{array}$ & 16 & \\
\hline IP-IPX & $\begin{array}{l}\text { Standard for the transmission of } 802.2 \text { packets } \\
\text { over IPX networks }\end{array}$ & 49 & \\
\hline-------- & $\begin{array}{l}\text { Requirements for Internet Hosts - Application } \\
\text { and Support }\end{array}$ & 3 & 1123 \\
\hline-------- & $\begin{array}{l}\text { Requirements for Internet Hosts - Communication } \\
\text { Layers }\end{array}$ & 3 & 1122 \\
\hline IGMP & Host extensions for IP multicasting & 5 & \\
\hline IP-NETBIOS & $\begin{array}{l}\text { Standard for the transmission of IP datagrams } \\
\text { over NetBIos networks }\end{array}$ & 48 & \\
\hline IP-SLIP & $\begin{array}{l}\text { Nonstandard for transmission of IP datagrams } \\
\text { over serial lines: SLIP }\end{array}$ & 47 & 1055 \\
\hline IP $-\mathrm{HC}$ & $\begin{array}{l}\text { Internet Protocol on Network System's } \\
\text { HYPERchannel: Protocol Specification }\end{array}$ & 45 & 1044 \\
\hline IP-IEEE & $\begin{array}{l}\text { Standard for the transmission of IP datagrams } \\
\text { over IEEE } 802 \text { networks }\end{array}$ & 43 & 1042 \\
\hline DOMAIN & Domain names - implementation and specification & 13 & 1035 \\
\hline DOMAIN & Domain names - concepts and facilities & 13 & 1034 \\
\hline $\mathrm{TP}-\mathrm{TCP}$ & $\begin{array}{l}\text { ISO Transport Service on top of the TCP Version: } \\
3\end{array}$ & 35 & 1006 \\
\hline NETBIOS & $\begin{array}{l}\text { Protocol standard for a NetBIOS service on a } \\
\text { TCP/UDP transport: Detailed specifications }\end{array}$ & 19 & 1002 \\
\hline NETBIOS & $\begin{array}{l}\text { Protocol standard for a NetBIOS service on a } \\
\text { TCP/UDP transport: Concepts and methods }\end{array}$ & 19 & 1001 \\
\hline FTP & File Transfer Protocol & 9 & 959 \\
\hline-------- & Internet Standard Subnetting Procedure & 5 & 950 \\
\hline-------- & $\begin{array}{l}\text { Broadcasting Internet datagrams in the presence } \\
\text { of subnets }\end{array}$ & 5 & 922 \\
\hline------- & Broadcasting Internet Datagrams & 5 & 919 \\
\hline IP-WB & Host Access Protocol specification & 40 & 907 \\
\hline RARP & A Reverse Address Resolution Protocol & 38 & 903 \\
\hline IP-EE & Standard for the transmission of IP datagrams & 42 & 895 \\
\hline
\end{tabular}




\begin{tabular}{|c|c|c|c|}
\hline$I P-E$ & $\begin{array}{l}\text { A Standard for the Transmission of IP Datagrams } \\
\text { over Ethernet Networks }\end{array}$ & 41 & 894 \\
\hline IP $-\mathrm{DC}$ & DCN Local-Network Protocols & 44 & 891 \\
\hline TIME & Time Protocol & 26 & 868 \\
\hline DAYTIME & Daytime Protocol & 25 & 867 \\
\hline USERS & Active users & 24 & 866 \\
\hline QUOTE & Quote of the Day Protocol & 23 & 865 \\
\hline CHARGEN & Character Generator Protocol & 22 & 864 \\
\hline DISCARD & Discard Protocol & 21 & 863 \\
\hline $\mathrm{ECHO}$ & Echo Protocol & 20 & 862 \\
\hline-------- & Telnet Status Option & 30 & 859 \\
\hline TELNET & Telnet Protocol Specification & 8 & 854 \\
\hline $\mathrm{ARP}$ & $\begin{array}{l}\text { Ethernet Address Resolution Protocol: Or } \\
\text { Converting Network Protocol Addresses to } 48 . \text { bit } \\
\text { Ethernet Address for Transmission on Ethernet } \\
\text { Hardware }\end{array}$ & 37 & 826 \\
\hline TCP & Transmission Control Protocol & 7 & 793 \\
\hline ICMP & Internet Control Message Protocol & 5 & 792 \\
\hline IP & Internet Protocol & 5 & 791 \\
\hline UDP & User Datagram Protocol & 6 & 768 \\
\hline
\end{tabular}

\subsection{Draft Standard Protocols}

[Note: an asterisk at the end of a line indicates a change from the previous edition of this document.]

\begin{tabular}{|c|c|c|}
\hline Mnemonic & Title & $\mathrm{RFC \#}$ \\
\hline IPv6-PPP & IP Version 6 over PPP & $5072 *$ \\
\hline-------- & Autonomous System Confederations for BGP & $5065 *$ \\
\hline-------- & LDP Specification & $5036 *$ \\
\hline-------- & $\begin{array}{l}\text { Privacy Extensions for Stateless Address } \\
\text { Autoconfiguration in IPv6 }\end{array}$ & 4941 * \\
\hline-------- & $\begin{array}{l}\text { Extensible Provisioning Protocol (EPP) Transport } \\
\text { Over TCP }\end{array}$ & 4934 * \\
\hline-------- & $\begin{array}{l}\text { Extensible Provisioning Protocol (EPP) Contact } \\
\text { Mapping }\end{array}$ & $4933 *$ \\
\hline-------- & Extensible Provisioning Protocol (EPP) Host Mapping & $4932 *$ \\
\hline-------- & $\begin{array}{l}\text { Extensible Provisioning Protocol (EPP) Domain Name } \\
\quad \text { Mapping }\end{array}$ & 4931 * \\
\hline-------- & Extensible Provisioning Protocol (EPP) & $4930 *$ \\
\hline IPV6-AUTO & IPv6 Stateless Address Autoconfiguration & $4862 *$ \\
\hline IPV6-ND & Neighbor Discovery for IP version 6 (IPv6) & 4861 * \\
\hline MEXT-BGP 4 & Multiprotocol Extensions for BGP-4 & $4760 *$ \\
\hline RMON-MIB & $\begin{array}{l}\text { Remote Network Monitoring Management Information } \\
\text { Base Version } 2\end{array}$ & 4502 * \\
\hline $\mathrm{BGP}-\mathrm{RR}$ & $\begin{array}{l}\text { BGP Route Reflection: An Alternative to Full Mesh } \\
\text { Internal BGP (IBGP) }\end{array}$ & $4456 *$ \\
\hline
\end{tabular}




\begin{tabular}{|c|c|}
\hline---- & $\begin{array}{l}\text { Internet Control Message Protocol (ICMPv6) for the } \\
\text { Internet Protocol Version 6 (IPv6) Specification }\end{array}$ \\
\hline-------- & Message Submission for Mail \\
\hline-------- & IP Version 6 Addressing Architecture \\
\hline $\mathrm{BGP}-4$ & A Border Gateway Protocol 4 (BGP-4) \\
\hline SMFAX-IM & A Simple Mode of Facsimile Using Internet Mail \\
\hline FFIF & $\begin{array}{l}\text { Tag Image File Format Fax extended (TIFF-FX) - } \\
\text { image/tiff-fx MIME Sub-type Registration }\end{array}$ \\
\hline FFIF & File Format for Internet Fax \\
\hline NICNAME & WHOIS Protocol Specification \\
\hline CONT-DUR & Content Duration MIME Header Definition \\
\hline MIME-ADPCM & $\begin{array}{l}\text { Toll Quality Voice - } 32 \mathrm{kbit} / \mathrm{s} \text { Adaptive Differential } \\
\text { Pulse Code Modulation (ADPCM) MIME Sub-type } \\
\text { Registration }\end{array}$ \\
\hline MIME-VP 2 & Voice Profile for Internet Mail - version 2 (VPIMv2) \\
\hline $\mathrm{EMF}-\mathrm{MDN}$ & Message Disposition Notification \\
\hline VRRP & Virtual Router Redundancy Protocol (VRRP) \\
\hline-------- & DNS Extensions to Support IP Version 6 \\
\hline-------- & $\begin{array}{l}\text { Textual Conventions for MIB Modules Using } \\
\text { Performance History Based on } 15 \text { Minute Intervals }\end{array}$ \\
\hline-------- & $\begin{array}{l}\text { Definitions of Managed objects for the Synchronous } \\
\text { Optical Network/Synchronous Digital Hierarchy } \\
\text { (SONET/SDH) Interface Type }\end{array}$ \\
\hline DSN & $\begin{array}{l}\text { An Extensible Message Format for Delivery Status } \\
\text { Notifications }\end{array}$ \\
\hline EMS-CODE & Enhanced Mail System Status Codes \\
\hline MIME-RPT & $\begin{array}{l}\text { The Multipart/Report Content Type for the Reporting } \\
\text { of Mail System Administrative Messages }\end{array}$ \\
\hline SMTP-DSN & $\begin{array}{l}\text { Simple Mail Transfer Protocol (SMTP) Service } \\
\text { Extension for Delivery Status Notifications (DSNs) }\end{array}$ \\
\hline-------- & Capabilities Advertisement with BGP-4 \\
\hline TIFF & $\begin{array}{l}\text { Tag Image File Format (TIFF) - image/tiff MIME } \\
\text { Sub-type Registration }\end{array}$ \\
\hline-------- & Content Language Headers \\
\hline-------- & $\begin{array}{l}\text { (Extensible Markup Language) XML-Signature Syntax } \\
\text { and Processing }\end{array}$ \\
\hline MINEAX-IM & Minimal FAX address format in Internet Mail \\
\hline MIN-PSTN & Minimal GSTN address format in Internet Mail \\
\hline RMON-MIB & $\begin{array}{l}\text { Remote Network Monitoring MIB Protocol Identifier } \\
\text { Reference }\end{array}$ \\
\hline RADIUS & Remote Authentication Dial In User Service (RADIUS) \\
\hline INTERGRMIB & The Interfaces Group MIB \\
\hline-------- & Host Resources MIB \\
\hline SNMP & $\begin{array}{l}\text { Definitions of Managed Objects for Extensible SNMP } \\
\text { Agents }\end{array}$ \\
\hline-------- & Agent Extensibility (AgentX) Protocol Version 1 \\
\hline-------- & HTTP Authentication: Basic and Digest Access \\
\hline
\end{tabular}




\begin{tabular}{|c|c|c|}
\hline HTTP & Hypertext Transfer Protocol -- HTTP/1.1 & 2616 \\
\hline-------- & $\begin{array}{l}\text { Remote Network Monitoring MIB Extensions for } \\
\text { Switched Networks Version } 1.0\end{array}$ & 2613 \\
\hline IPV 6 & Internet Protocol, Version 6 (IPv6) Specification & 460 \\
\hline IARP & Inverse Address Resolution Protocol & 2390 \\
\hline TN3270E & TN3270 Enhancements & 2355 \\
\hline TFTP-Opt & TFTP Timeout Interval and Transfer Size Options & \\
\hline $\mathrm{TFTP}-\mathrm{Blk}$ & TFTP Blocksize Option & 2348 \\
\hline TFTP-Ext & TFTP Option Extension & \\
\hline DHCP-BOOTP & DHCP Options and BOOTP Vendor Extensions & \\
\hline $\mathrm{DHCP}$ & Dynamic Host Configuration Protocol & \\
\hline FRAME-MIB & $\begin{array}{l}\text { Management Information Base for Frame Relay DTEs } \\
\text { Using SMIv2 }\end{array}$ & \\
\hline IP-HIPP I & IP over HIPPI & \\
\hline MIME-CONF & $\begin{array}{l}\text { Multipurpose Internet Mail Extensions (MIME) Part } \\
\text { Five: Conformance Criteria and Examples }\end{array}$ & \\
\hline MIME-MSG & $\begin{array}{l}\text { MIME (Multipurpose Internet Mail Extensions) Part } \\
\text { Three: Message Header Extensions for Non-ASCII Text }\end{array}$ & 2047 \\
\hline MIME-MEDIA & $\begin{array}{l}\text { Multipurpose Internet Mail Extensions (MIME) Part } \\
\text { Two: Media Types }\end{array}$ & 2046 \\
\hline MIME & $\begin{array}{l}\text { Multipurpose Internet Mail Extensions (MIME) Part } \\
\text { One: Format of Internet Message Bodies }\end{array}$ & 2045 \\
\hline $\mathrm{PPP}-\mathrm{CHAP}$ & $\begin{array}{l}\text { PPP Challenge Handshake Authentication Protocol } \\
\text { (CHAP) }\end{array}$ & 1994 \\
\hline $\mathrm{PPP}-\mathrm{MP}$ & The PPP Multilink Protocol (MP) & \\
\hline PPP-LINK & PPP Link Quality Monitoring & 1989 \\
\hline MTU-IPV6 & Path MTU Discovery for IP version 6 & 1981 \\
\hline $\mathrm{CON}-\mathrm{MD} 5$ & The Content-MD5 Header Field & 1864 \\
\hline $\mathrm{BGP}-4-\mathrm{APP}$ & $\begin{array}{l}\text { Application of the Border Gateway Protocol in the } \\
\text { Internet }\end{array}$ & 1772 \\
\hline PPP - DNCP & The PPP DECnet Phase IV Control Protocol (DNCP) & 1762 \\
\hline $802.5-\mathrm{MIB}$ & IEEE 802.5 MIB using SMIv2 & 1748 \\
\hline RIP 2-MIB & RIP Version 2 MIB Extension & 1724 \\
\hline SIP-MIB & $\begin{array}{l}\text { Definitions of Managed Objects for SMDS Interfaces } \\
\text { using SMIv2 }\end{array}$ & 1694 \\
\hline ----ー--- & $\begin{array}{l}\text { Definitions of Managed Objects for } \\
\text { Parallel-printer-like Hardware Devices using SMIv2 }\end{array}$ & 1660 \\
\hline -------- & $\begin{array}{l}\text { Definitions of Managed Objects for RS-232-like } \\
\text { Hardware Devices using SMIv2 }\end{array}$ & 1659 \\
\hline-------- & $\begin{array}{l}\text { Definitions of Managed Objects for Character stream } \\
\text { Devices using SMIv2 }\end{array}$ & 1658 \\
\hline-------- & SMTP Service Extension for 8bit-MIMEtransport & \\
\hline OSI-NSAP & Guidelines for OSI NSAP Allocation in the Internet & 1629 \\
\hline ISO-TS-ECH & An Echo Function for CLNP (ISO 8473) & 1575 \\
\hline DECNET-MIB & DECnet Phase IV MIB Extensions & 1559 \\
\hline--------- & $\begin{array}{l}\text { Clarifications and Extensions for the Bootstrap } \\
\text { Protocol }\end{array}$ & 1542 \\
\hline DHCP-BOOTP & Interoperation Between DHCP and BOOTP & 1534 \\
\hline
\end{tabular}


IP-X.25 Multiprotocol Interconnect on X.25 and ISDN in the Packet Mode

NTPV3 Network Time Protocol (Version 3) Specification, Implementation and Analysis

$\begin{array}{llr}\text { FINGER } & \text { The Finger User Information Protocol } & 1288 \\ \text { IP-MTU } & \text { Path MTU discovery } & 1191\end{array}$

------ Proposed Standard for the Transmission of IP 1188

TOPT-LINE Telnet Linemode Option 1184

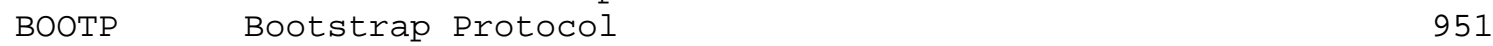

\subsection{Proposed Standard Protocols}

[Note: an asterisk at the end of a line indicates a change from the previous edition of this document.]

\begin{tabular}{|c|c|c|}
\hline Mnemonic & Title & RFC\# \\
\hline-------- & $\begin{array}{l}\text { Sieve Email Filtering: Spamtest and Virustest } \\
\text { Extensions }\end{array}$ & $5235 *$ \\
\hline-------- & Sieve Email Filtering: Subaddress Extension & $5233 *$ \\
\hline-------- & Sieve Email Filtering: Imap4flags Extension & 5232 * \\
\hline-------- & Sieve Email Filtering: Relational Extension & 5231 * \\
\hline-------- & Sieve Email Filtering: Vacation Extension & $5230 *$ \\
\hline-------- & Sieve Email Filtering: Variables Extension & $5229 *$ \\
\hline-------- & Sieve: An Email Filtering Language & $5228 *$ \\
\hline-------- & A More Loss-Tolerant RTP Payload Format for MP3 Audio & $5219 *$ \\
\hline-------- & Mobility Header Home Agent Switch Message & $5142 *$ \\
\hline-------- & $\begin{array}{l}\text { Terminal Endpoint Identifier (TEI) Query Request } \\
\text { Number Change }\end{array}$ & $5133 *$ \\
\hline-------- & IP Multicast MIB & $5132 *$ \\
\hline-------- & A MIB Textual Convention for Language Tags & 5131 * \\
\hline-------- & $\begin{array}{l}\text { A Policy Control Mechanism in IS-IS Using } \\
\text { Administrative Tags }\end{array}$ & 5130 * \\
\hline-------- & Explicit Congestion Marking in MPLS & $5129 *$ \\
\hline-------- & $\begin{array}{l}\text { Transmission of IPv6 via the IPv6 Convergence } \\
\text { Sublayer over IEEE } 802.16 \text { Networks }\end{array}$ & 5121 * \\
\hline-------- & $\begin{array}{l}\text { M-ISIS: Multi Topology (MT) Routing in Intermediate } \\
\text { System to Intermediate Systems (IS-ISS) }\end{array}$ & $5120 *$ \\
\hline-------- & $\begin{array}{l}\text { An Interface and Algorithms for Authenticated } \\
\text { Encryption }\end{array}$ & $5116 *$ \\
\hline-------- & $\begin{array}{l}\text { Telephony Routing over IP (TRIP) Attribute for } \\
\text { Resource Priority }\end{array}$ & $5115 *$ \\
\hline-------- & $\begin{array}{l}\text { The Presence-Specific Static Dictionary for } \\
\text { Signaling Compression (Sigcomp) }\end{array}$ & 5112 * \\
\hline -------- & $\begin{array}{l}\text { RTP Payload Format for Generic Forward Error } \\
\text { Correction }\end{array}$ & $5109 *$ \\
\hline-------- & DHCP Server Identifier Override Suboption & $5107 *$ \\
\hline
\end{tabular}




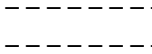

$-------$

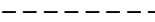

$-------$

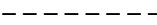

$--------$

$--------$

IMAP-URL
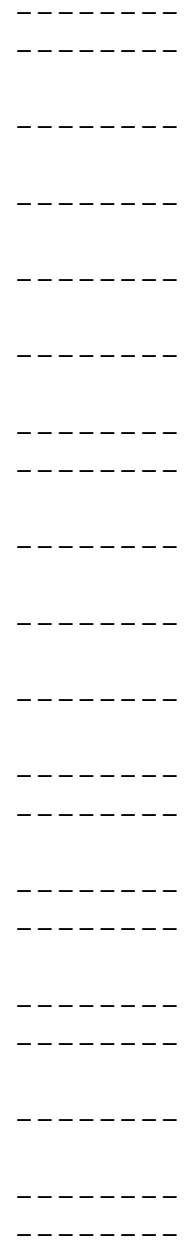

- - - - - - -

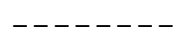

RFC Editor
ENUM Validation Token Format Definition

$5105 *$

Bidirectional Flow Export Using IP Flow Information Export (IPFIX)

Information Model for IP Flow Information Export

Specification of the IP Flow Information Export

(IPFIX) Protocol for the Exchange of IP Traffic Flow Information

MIB for the UDP-Lite protocol

Mobile IPv6 Experimental Messages

Deprecation of Type 0 Routing Headers in IPv6

Mobile IPv6 Vendor Specific Option

IMAP URL Scheme

RADIUS Extension for Digest Authentication

IS-IS Protocol Extensions for Path Computation Element ( $\mathrm{PCE}$ ) Discovery

OSPF Protocol Extensions for Path Computation Element ( $\mathrm{PCE})$ Discovery

Pseudowire Virtual Circuit Connectivity Verification (VCCV): A Control Channel for Pseudowires

Using AES-CCM and AES-GCM Authenticated Encryption in the Cryptographic Message Syntax (CMS)

Cryptographic Message Syntax (CMS) Authenticated-Enveloped-Data Content Type

The Generalized TTL Security Mechanism (GTSM)

Common Remote Authentication Dial In User Service (RADIUS) Implementation Issues and Suggested Fixes

Rejecting Anonymous Requests in the Session Initiation Protocol (SIP)

Transport Layer Security (TLS) Session Resumption without Server-Side state

ENUM Validation Information Mapping for the Extensible Provisioning Protocol

IPv6 Router Advertisement Flags Option

IGP Routing Protocol Extensions for Discovery of Traffic Engineering Node Capabilities

The Incident Object Description Exchange Format

Ethernet in the First Mile Copper (EFMCu) Interfaces MIB

The Archived-At Message Header Field

Extensions to GMPLS Resource Reservation Protocol (RSVP) Graceful Restart

Stream Control Transmission Protocol (SCTP) Dynamic Address Reconfiguration

Protocol Independent Multicast MIB

Bootstrap Router (BSR) Mechanism for Protocol Independent Multicast (PIM)

On the Use of Channel Bindings to Secure Channels

Server-Based Certificate Validation Protocol (SCVP)
$5103 *$

$5102 *$

$5101 *$

$5097 *$

$5096 *$

$5095 *$

5094 *

$5092 *$

$5090 *$

$5089 *$

5088 *

$5085 *$

5084 *

$5083 *$

$5082 *$

$5080 *$

$5079 *$

$5077 *$

$5076^{*}$

$5075 *$

$5073 *$

$5070 *$

$5066 *$

5064 *

$5063 *$

5061 *

$5060 *$

$5059 \star$

$5056 *$

$5055 *$ 


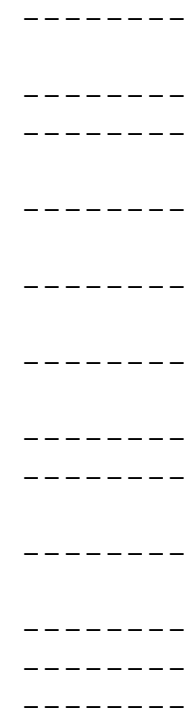

POP 3-AUTH
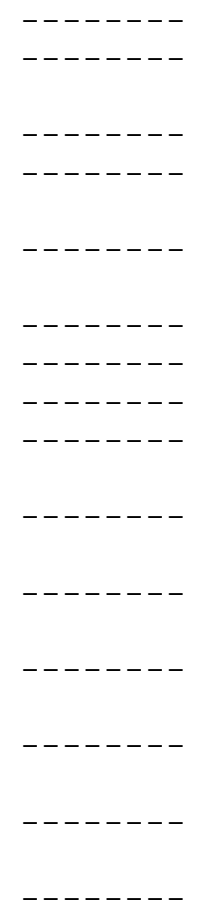

Raptor Forward Error Correction Scheme for object Delivery

Forward Error Correction (FEC) Building Block

i; unicode-casemap - Simple Unicode Collation Algorithm

Applying Signaling Compression (SigComp) to the Session Initiation Protocol (SIP)

Internet Small Computer System Interface (iSCSI) 5048* Corrections and Clarifications

Internet Small Computer system Interface (iSCSI)

Extensions for Remote Direct Memory Access (RDMA)

Marker PDU Aligned Framing for TCP Specification

Stream Control Transmission Protocol (SCTP) Direct Data Placement (DDP) Adaptation

Direct Data Placement Protocol (DDP) / Remote Direct Memory Access Protocol (RDMAP) Security

Direct Data Placement over Reliable Transports

A Remote Direct Memory Access Protocol Specification

Enhanced Security Services (ESS) Update: Adding CertID Algorithm Agility

The Post Office Protocol (POP3) Simple Authentication and Security Layer (SASL) Authentication Mechanism

WITHIN Search Extension to the IMAP Protocol

A Uniform Resource Name (URN) for Emergency and Other Well-known Services

Definition of an IS-IS Link Attribute Sub-TLV 5029*

A Telephone Number Mapping (ENUM) Service 5028* Registration for Instant Messaging (IM) Services

Security Preconditions for Session Description 5027* Protocol (SDP) Media Streams

Mobile IPv6 Bootstrapping in Split Scenario 5026*

Presence Authorization Rules 5025*

The Atom Publishing Protocol 5023*

Extended Kerberos Version 5 Key Distribution Center 5021* (KDC) Exchanges over TCP

The Lightweight Directory Access Protocol (LDAP) entryDN Operational Attribute

The Lightweight Online Certificate status Protocol (OCSP) Profile for High-Volume Environments

Connection Establishment in the Binary Floor Control 5018* Protocol (BFCP)

MIB Textual Conventions for Uniform Resource 5017* Identifiers (URIs)

Bidirectional Protocol Independent Multicast 5015* (BIDIR-PIM)

Automated Updates of DNS Security (DNSSEC) Trust 5011* Anchors 


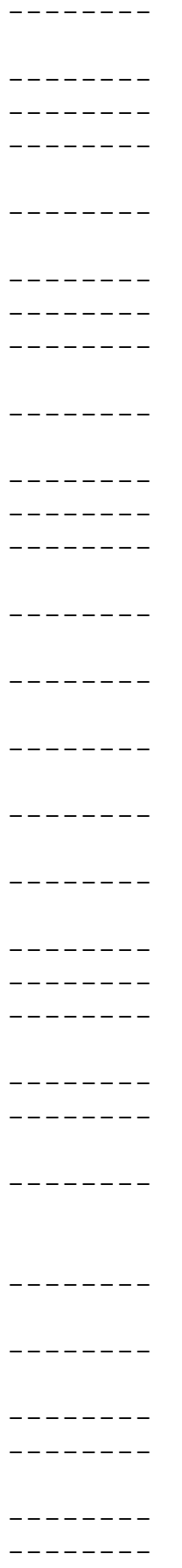

The Dynamic Host Configuration Protocol Version 4

$5010 *$

(DHCPv4) Relay Agent Flags Suboption

DHCPv6 Leasequery

$5007 *$

Feed Paging and Archiving

$5005 *$

Avoid BGP Best Path Transitions from One External to

$5004 *$ Another

Attachment Individual Identifier (AII) Types for

$5003 *$

Aggregation

DNS Name Server Identifier (NSID) Option

Evidence Record Syntax (ERS)

$5001 *$

Formal Notation for Robust Header Compression

$4998 *$ ( $\mathrm{ROHC}-\mathrm{FN}$ )

RObust Header Compression (ROHC): A Profile for $\mathrm{TCP} / \mathrm{IP}$ (ROHC-TCP)

The RObust Header Compression (ROHC) Framework 4995*

DHCPv6 Relay Agent Echo Request Option 4994*

A Lightweight UDP Transfer Protocol for the Internet 4993* Registry Information Service

XML Pipelining with Chunks for the Internet Registry 4992* Information Service

A Common Schema for Internet Registry Information 4991* Service Transfer Protocols

Internet X.509 Public Key Infrastructure Subject Alternative Name for Expression of Service Name

Fibre Channel Registered State Change Notification (RSCN) MIB

Support for Multiple Hash Algorithms in Cryptographically Generated Addresses (CGAs)

IANA Registration for Enumservice 'XMPP'

4985 *

The IMAP COMPRESS Extension

Relay Extensions for the Message Sessions Relay Protocol (MSRP)

The Message Session Relay Protocol (MSRP)

Generalized MPLS (GMPLS) RSVP-TE Signaling Extensions in Support of Calls

Routing Extensions for Discovery of Multiprotocol (MPLS) Label Switch Router (LSR) Traffic Engineering (TE) Mesh Membership

Intermediate System to Intermediate System (IS-IS) 4971* Extensions for Advertising Router Information

Extensions to OSPF for Advertising Optional Router 4970* Capabilities

IANA Registration for vCard Enumservice 4969*

Dial String Parameter for the Session Initiation 4967* Protocol Uniform Resource Identifier

Stream Control Transmission Protocol 4960*

IMAP Extension for Simple Authentication and 4959* Security Layer (SASL) Initial Client Response 


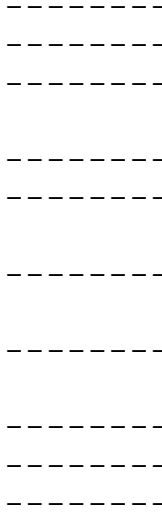

WEBDAV
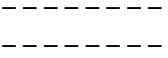

-------
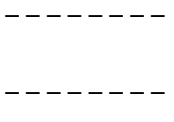

- - - - - -
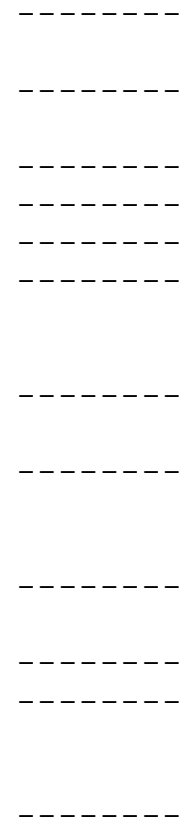

DNS Security (DNSSEC) Experiments

SMTP Service Extension for Authentication

Fail Over Extensions for Layer 2 Tunneling Protocol (L2TP) "failover"

ICMP Extensions for Multiprotocol Label Switching

The Internet IP Security PKI Profile of IKEV1/ISAKMP, IKEV2, and PKIX

Transmission of IPv6 Packets over IEEE 802.15.4 Networks

Definitions of Managed Objects for isNS (Internet Storage Name Service)

Fibre Channel Zone Server MIB

Fibre Channel Fabric Configuration Server MIB

Crankback Signaling Extensions for MPLS and GMPLS RSVP-TE

HTTP Extensions for Web Distributed Authoring and Versioning (WebDAV)

Mobile IPv4 Message String Extension

Connected Identity in the Session Initiation Protocol (SIP)

Multi-Topology (MT) Routing in OSPF

Representing Trunk Groups in tel/sip Uniform Resource Identifiers (URIs)

Protocol Extensions for Header Compression over MPLS TCP Extended Statistics MIB

Signaling Compression (SigComp) Corrections and Clarifications

Authenticated Chunks for the Stream Control Transmission Protocol (SCTP)

BGP Support for Four-octet AS Number space

Extended ICMP to Support Multi-Part Messages

OpenPGP Message Format

Definitions and Managed Objects for Operations, Administration, and Maintenance (OAM) Functions on Ethernet-Like Interfaces

Mobile IPv6 Operation with IKEv2 and the Revised IPsec Architecture

Extensions to Resource Reservation Protocol Traffic Engineering (RSVP-TE) for

Point-to-Multipoint TE Label Switched Paths (LSPS)

Exclude Routes - Extension to Resource ReserVation Protocol-Traffic Engineering (RSVP-TE)

GMPLS Segment Recovery

RSVP-TE Extensions in Support of End-to-End Generalized Multi-Protocol Label Switching (GMPLS) Recovery

Domainkeys Identified Mail (DKIM) Signatures
$4955 *$

4954 *

$4951 *$

4950 *

$4945 *$

4944 *

4939*

$4936 *$

$4935 *$

$4920 *$

4918 *

$4917 *$

$4916 *$

$4915 *$

4904 *

4901 *

$4898 *$

$4896 *$

$4895 *$

$4893 *$

4884 *

$4880 *$

$4878 *$

4877 *

$4875 *$

4874 *

4873*

4872 *

4871 * 

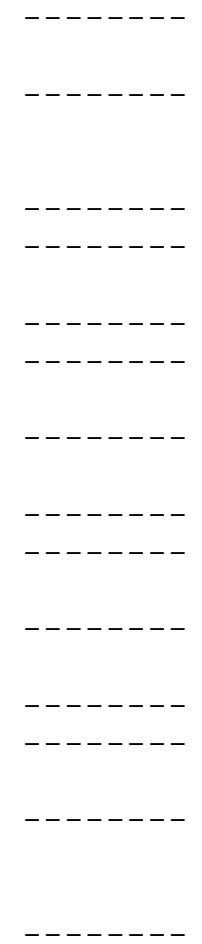

MAU-MIB

ESP
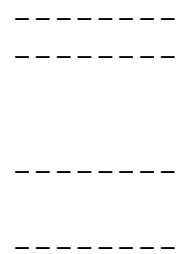

RIP 2-MD 5
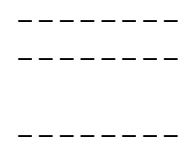

$-------$ $-------$
Using HMAC-SHA-256, HMAC-SHA-384, and HMAC-SHA-512 with IPsec

RTP Payload Format and File Storage Format for the Adaptive Multi-Rate (AMR) and Adaptive Multi-Rate Wideband (AMR-WB) Audio Codecs

Enhanced Route Optimization for Mobile IPv6

SMTP Submission Service Extension for Future Message Release

Wildcard Pseudowire Type

Generic Aggregate Resource ReSerVation Protocol (RSVP) Reservations

Media Type Registration of Payload Formats in the RTP Profile for Audio and Video Conferences

Media Type Registration of RTP Payload Formats

Cryptographic Message Syntax (CMS) Multiple Signer Clarification

Declarative Public Extension Key for Internet Small Computer Systems Interface (iSCSI) Node Architecture

RADIUS Filter Rule Attribute

Domain-Based Application Service Location Using URIs and the Dynamic Delegation Discovery Service (DDDS)

Synchronous Optical Network/Synchronous Digital Hierarchy (SONET/SDH) Circuit Emulation over Packet (CEP)

Managed Objects of Ethernet Passive Optical Networks (EPON)

Definitions of Managed Objects for IEEE 802.3 Medium Attachment Units (MAUs)

Cryptographic Algorithm Implementation Requirements for Encapsulating Security Payload (ESP) and Authentication Header ( $\mathrm{AH})$

Timezone Options for DHCP

An Extensible Markup Language (XML) Configuration Access Protocol (XCAP) Usage for Manipulating Presence Document Contents

Extensible Markup Language (XML) Formats for Representing Resource Lists

The Extensible Markup Language (XML) Configuration Access Protocol (XCAP)

RIPv2 Cryptographic Authentication

Packetization Layer Path MTU Discovery

Padding Chunk and Parameter for the Stream Control Transmission Protocol (SCTP)

Secure Shell Public Key Subsystem

RADIUS Delegated-IPv6-Prefix Attribute

Encapsulation of MPLS over Layer 2 Tunneling Protocol Version 3
4868 *

4867 *

4866 *

$4865 *$

$4863 *$

4860 *

4856 *

4855 *

$4853 *$

4850 *

$4849 *$

$4848 *$

4842 *

4837 *

4836 *

$4835 *$

4833 *

$4827 *$

4826 *

4825 *

4822 *

4821 *

4820 *

$4819 *$

$4818 *$

4817 *

RFC Editor

Informational

[Page 16] 

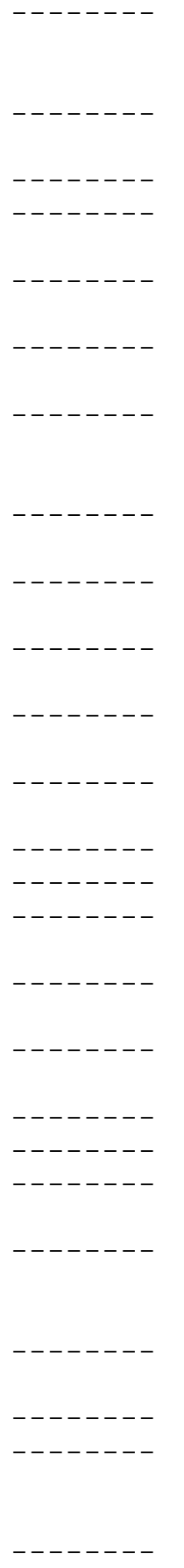

Pseudowire Emulation Edge-to-Edge (PWE3) Asynchronous Transfer Mode (ATM) Transparent Cell Transport Service

RObust Header Compression (ROHC): Corrections and Clarifications to RFC 3095

IPsec Security Policy Database Configuration MIB 4807* Online Certificate Status Protocol (OCSP) Extensions 4806* to IKEv2

Definitions of Managed Objects for the DS1, J1, E1, 4805* DS2, and E2 Interface Types

Aggregation of Resource ReSerVation Protocol (RSVP) 4804* Reservations over MPLS TE/DS-TE Tunnels

Generalized Multiprotocol Label Switching (GMPLS) 4803* Label Switching Router (LSR) Management Information Base

Generalized Multiprotocol Label Switching (GMPLS) Traffic Engineering Management Information Base

Definitions of Textual Conventions for Generalized Multiprotocol Label Switching (GMPLS) Management Connecting IPv6 Islands over IPv4 MPLS Using IPv6 Provider Edge Routers (6PE)

The Session Description Protocol (SDP) Content Attribute

Encoding Instructions for the Generic String Encoding Rules (GSER)

Calendaring Extensions to WebDAV (CalDAV)

Internet Application Protocol Collation Registry

Simple Network Management Protocol (SNMP) over IEEE 802 Networks

Enhancements to RTP Payload Formats for EVRC Family Codecs

Pre-Shared Key (PSK) Ciphersuites with NULL Encryption for Transport Layer Security (TLS)

GMPLS - Communication of Alarm Information

Graceful Restart Mechanism for BGP with MPLS

Management Information Base for the Session Initiation Protocol (SIP)

Dynamic Host Configuration Protocol (DHCPv4 and DHCPv6) Option for Civic Addresses Configuration Information

Integrity Transform Carrying Roll-Over Counter for the Secure Real-time Transport Protocol (SRTP)

vCard Extensions for Instant Messaging (IM)

IANA Registration for an Enumservice Containing Public Switched Telephone Network (PSTN) Signaling Information

Virtual Private LAN Service (VPLS) Using Label Distribution Protocol (LDP) Signaling
$4816 *$

$4815 *$

$4807 *$
$4806 *$

4802 *

$4801 *$

$4798 *$

$4796 *$

4792 *

$4791 *$

4790 *

4789*

4788 *

$4785 *$

4783 *

4781 *

4780 *

4776 *

4771 *

4770 *

4769 *

4762 *

[Page 17]

RFC Editor 


\begin{tabular}{|c|c|c|}
\hline-------- & $\begin{array}{l}\text { Virtual Private LAN Service (VPLS) Using BGP for } \\
\text { Auto-Discovery and Signaling }\end{array}$ & 476 \\
\hline-------- & The ENUM Dip Indicator Parameter for the "tel" URI & $4759 *$ \\
\hline----- & $\begin{array}{l}\text { Forward Error Correction Grouping Semantics in } \\
\text { Session Description Protocol }\end{array}$ & 4756 * \\
\hline-------- & IP over InfiniBand: Connected Mode & $4755 *$ \\
\hline-------- & $\begin{array}{l}\text { IKE and IKEv2 Authentication Using the Elliptic } \\
\text { Curve Digital Signature Algorithm (ECDSA) }\end{array}$ & 4754 * \\
\hline SASL & $\begin{array}{l}\text { The Kerberos V5 ("GSSAPI") Simple Authentication and } \\
\text { Security Layer (SASL) Mechanism }\end{array}$ & 4752 * \\
\hline OSPE-MIB & OSPF Version 2 Management Information Base & $4750 *$ \\
\hline-------- & RTP Payload Format for the G.729.1 Audio Codec & $4749 *$ \\
\hline-------- & The Virtual Fabrics MIB & 4747 * \\
\hline-------- & $\begin{array}{l}\text { Common Policy: A Document Format for Expressing } \\
\text { Privacy Preferences }\end{array}$ & $4745 *$ \\
\hline-------- & $\begin{array}{l}\text { Using the NETCONF Protocol over the Blocks } \\
\text { Extensible Exchange Protocol (BEEP) }\end{array}$ & 4744 * \\
\hline-------- & $\begin{array}{l}\text { Using NETCONF over the Simple Object Access Protocol } \\
\text { (SOAP) }\end{array}$ & 4743 * \\
\hline-------- & $\begin{array}{l}\text { Using the NETCONF Configuration Protocol over Secure } \\
\text { SHell (SSH) }\end{array}$ & 4742 * \\
\hline-------- & NETCONF Configuration Protocol & 4741 * \\
\hline-------- & $\begin{array}{l}\text { Diameter Session Initiation Protocol (SIP) } \\
\text { Application }\end{array}$ & $4740 *$ \\
\hline------ & $\begin{array}{l}\text { MIKEY-RSA-R: An Additional Mode of Key Distribution } \\
\text { in Multimedia Internet KEYing (MIKEY) }\end{array}$ & 4738 * \\
\hline-------- & Packet Reordering Metrics & 4737 * \\
\hline-------- & Example Media Types for Use in Documentation & $4735 *$ \\
\hline-------- & $\begin{array}{l}\text { Definition of Events for Modem, Fax, and Text } \\
\text { Telephony Signals }\end{array}$ & 4734 * \\
\hline-------- & $\begin{array}{l}\text { RTP Payload for DTMF Digits, Telephony Tones, and } \\
\text { Telephony Signals }\end{array}$ & $4733 *$ \\
\hline-------- & $\begin{array}{l}\text { IMAP } 4 \text { Extension to SEARCH Command for Controlling } \\
\text { What Kind of Information Is Returned }\end{array}$ & 4731 * \\
\hline-------- & $\begin{array}{l}\text { A Session Initiation Protocol (SIP) Event Package } \\
\text { for Key Press Stimulus (KPML) }\end{array}$ & 4730 * \\
\hline-------- & $\begin{array}{l}\text { Experimental Values In IPv4, IPv6, ICMPv4, ICMPv6, } \\
\text { UDP, and TCP Headers }\end{array}$ & 4727 * \\
\hline-------- & Graceful Restart Mechanism for BGP & 4724 * \\
\hline-------- & Mobile IPv4 Challenge/Response Extensions (Revised) & 4721 * \\
\hline-------- & $\begin{array}{l}\text { Pseudowire Emulation Edge-to-Edge (PWE3) Frame Check } \\
\text { Sequence Retention }\end{array}$ & 4720 * \\
\hline-------- & $\begin{array}{l}\text { Transport of Ethernet Frames over Layer } 2 \text { Tunneling } \\
\text { Protocol Version } 3 \text { (L2TPv3) }\end{array}$ & $4719 *$ \\
\hline-------- & $\begin{array}{l}\text { Encapsulation Methods for Transport of Asynchronous } \\
\text { Transfer Mode (ATM) over MPLS Networks }\end{array}$ & 4717 * \\
\hline
\end{tabular}




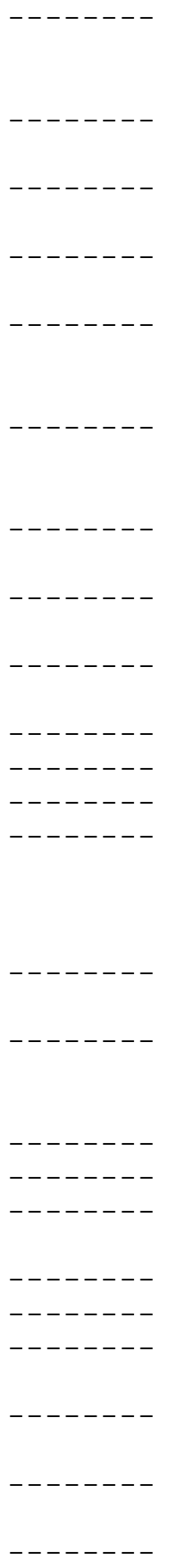

Transport Mappings for Real-time Application

4712 * Quality-of-Service Monitoring (RAQMON) Protocol Data Unit (PDU)

Real-time Application Quality-of-Service Monitoring (RAQMON) MIB

Real-time Application Quality-of-Service Monitoring (RAQMON) Framework Definitions of Managed Objects for Asymmetric 4706* Digital Subscriber Line 2 (ADSL2)

The Dynamic Host Configuration Protocol for IPv6 (DHCPv6) Client Fully Qualified Domain Name (FQDN) Option

Resolution of Fully Qualified Domain Name (FQDN) $4711 *$ 4710 * 4704 * Conflicts among Dynamic Host Configuration Protocol (DHCP) Clients

The Dynamic Host Configuration Protocol (DHCP) Client Fully Qualified Domain Name (FQDN) Option

A DNS Resource Record (RR) for Encoding Dynamic Host Configuration Protocol (DHCP) Information (DHCID RR) IRIS: An Address Registry (areg) Type for the Internet Registry Information Service

RTP Payload Format for MIDI

Number Portability Parameters for the "tel" URI

Atom Threading Extensions

Constrained Route Distribution for Border Gateway Protocol/Multiprotocol Label switching (BGP/MPLS) Internet Protocol (IP) Virtual Private Networks (VPNs)

Internet X.509 Public Key Infrastructure Subject Identification Method (SIM)

Multimedia Terminal Adapter (MTA) Management Information Base for PacketCable- and IPCablecom-Compliant Devices

TLS User Mapping Extension

TLS Handshake Message for Supplemental Data

RADIUS Attributes for Virtual LAN and Priority Support

RADIUS Authentication Server MIB for IPv6

RADIUS Authentication Client MIB for IPv6

Layer 2 Virtual Private Network (L2VPN) Extensions for Layer 2 Tunneling Protocol (L2TP)

Signaling System 7 (SS7) Message Transfer Part 3 4666* (MTP3) - User Adaptation Layer (M3UA)

A Session Initiation Protocol (SIP) Event Notification Extension for Resource Lists

$4703 *$

4701 *

4698 *

$4695 *$

4694 *

$4685 *$

4684 *

An Extensible Markup Language (XML)-Based Format for 4661* Event Notification Filtering 


\begin{tabular}{|c|c|c|}
\hline-------- & $\begin{array}{l}\text { Functional Description of Event Notification } \\
\text { Filtering }\end{array}$ & 4660 * \\
\hline-- & $\begin{array}{l}\text { BGP-MPLS IP Virtual Private Network (VPN) Extension } \\
\text { for IPV6 VPN }\end{array}$ & $4659 *$ \\
\hline-- & A One-way Active Measurement Protocol (OWAMP) & 4656 * \\
\hline--- & $\begin{array}{l}\text { HMAC-Authenticated Diffie-Hellman for Multimedia } \\
\text { Internet KEYing (MIKEY) }\end{array}$ & 4650 * \\
\hline-- & $\begin{array}{l}\text { Dynamic Host Configuration Protocol for IPv6 } \\
\text { (DHCPv6) Relay Agent Remote-ID Option }\end{array}$ & $4649 *$ \\
\hline-------- & The Base16, Base 32 , and Base 64 Data Encodings & $4648 *$ \\
\hline--- & $\begin{array}{l}\text { Network News Transfer Protocol (NNTP) Extension for } \\
\text { Streaming Feeds }\end{array}$ & 4644 * \\
\hline----- & $\begin{array}{l}\text { Network News Transfer Protocol (NNTP) Extension for } \\
\text { Authentication }\end{array}$ & $4643 *$ \\
\hline-------- & $\begin{array}{l}\text { Using Transport Layer Security (TLS) with Network } \\
\text { News Transfer Protocol (NNTP) }\end{array}$ & $4642 *$ \\
\hline-------- & $\begin{array}{l}\text { Cable Device Management Information Base for } \\
\text { Data-Over-Cable Service Interface Specification } \\
\text { (DocSIS) Compliant Cable Modems and Cable Modem } \\
\text { Termination Systems }\end{array}$ & $4639 *$ \\
\hline---- & Foreign Agent Error Extension for Mobile IPv4 & $4636 *$ \\
\hline-------- & $\begin{array}{l}\text { HMAC SHA (Hashed Message Authentication Code, Secure } \\
\text { Hash Algorithm) TSIG Algorithm Identifiers }\end{array}$ & $4635 *$ \\
\hline-------- & $\begin{array}{l}\text { Link Management Protocol (LMP) Management } \\
\text { Information Base (MIB) }\end{array}$ & 4631 * \\
\hline----- & $\begin{array}{l}\text { Update to Directorystring Processing in the Internet } \\
\text { X.509 Public Key Infrastructure Certificate and } \\
\text { Certificate Revocation List (CRL) Profile }\end{array}$ & $4630 *$ \\
\hline---- & RTP Payload Format for ITU-T Rec. H.263 Video & $4629 *$ \\
\hline--- & $\begin{array}{l}\text { MIB for Fibre Channel's Fabric Shortest Path First } \\
\text { (FSPF) Protocol }\end{array}$ & $4626 *$ \\
\hline-------- & Fibre Channel Routing Information MIB & $4625 *$ \\
\hline----- & $\begin{array}{l}\text { Pseudowire Emulation Edge-to-Edge (PWE3) } \\
\text { Fragmentation and Reassembly }\end{array}$ & 4623 * \\
\hline--- & $\begin{array}{l}\text { Internationalized Resource Identifiers (IRIs) and } \\
\text { Uniform Resource Identifiers (URIs) for the } \\
\text { Extensible Messaging and Presence Protocol (XMPP) }\end{array}$ & $4622 *$ \\
\hline & $\begin{array}{l}\text { Encapsulation Methods for Transport of Frame Relay } \\
\text { over Multiprotocol Label Switching (MPLS) Networks }\end{array}$ & $4619 *$ \\
\hline & $\begin{array}{l}\text { Encapsulation Methods for Transport of } \\
\text { PPP/High-Level Data Link Control (HDLC) over MPLS } \\
\text { Networks }\end{array}$ & 4618 * \\
\hline-------- & $\begin{array}{l}\text { The PLAIN Simple Authentication and Security Layer } \\
\text { (SASL) Mechanism }\end{array}$ & $4616 *$ \\
\hline-------- & $\begin{array}{l}\text { The Advanced Encryption Standard-Cipher-based } \\
\text { Message Authentication Code-Pseudo-Random } \\
\text { Function-128 (AES-CMAC-PRF-128) Algorithm for the } \\
\text { Internet Key Exchange Protocol (IKE) }\end{array}$ & $4615 *$ \\
\hline
\end{tabular}




\begin{tabular}{|c|c|c|}
\hline & Anycast-RP Using Protocol Independent Multicast (PIM) & 4610 * \\
\hline--- & Source-Specific Multicast for IP & $4607 *$ \\
\hline--- & $\begin{array}{l}\text { Generalized Multi-Protocol Label Switching (GMPLS) } \\
\text { Extensions for Synchronous optical Network (SONET) } \\
\text { and Synchronous Digital Hierarchy (SDH) Control }\end{array}$ & $4606 *$ \\
\hline-------- & $\begin{array}{l}\text { Internet Group Management Protocol (IGMP) / } \\
\text { Multicast Listener Discovery (MLD)-Based Multicast } \\
\text { Forwarding ("IGMP/MLD Proxying") }\end{array}$ & $4605 *$ \\
\hline-------- & $\begin{array}{l}\text { Using Internet Group Management Protocol Version } 3 \\
\text { (IGMPv3) and Multicast Listener Discovery Protocol } \\
\text { Version 2 (MLDv2) for Source-Specific Multicast }\end{array}$ & 4604 * \\
\hline $\mathrm{P} I \mathrm{M}-\mathrm{SM}$ & $\begin{array}{l}\text { Protocol Independent Multicast - Sparse Mode } \\
\text { (PIM-SM) : Protocol Specification (Revised) }\end{array}$ & 4601 * \\
\hline-------- & $\begin{array}{l}\text { Real-time Transport Protocol (RTP) Payload Format } \\
\text { for Enhanced AC-3 (E-AC-3) Audio }\end{array}$ & $4598 *$ \\
\hline-------- & The Role of Wildcards in the Domain Name System & 4592 * \\
\hline-------- & $\begin{array}{l}\text { Frame Relay over Layer } 2 \text { Tunneling Protocol Version } \\
\quad 3 \text { (L2TPv3) }\end{array}$ & 591 * \\
\hline-------- & Location Types Registry & $4589 *$ \\
\hline-------- & RTP Retransmission Payload Format & $4588 *$ \\
\hline RTP-H. 261 & RTP Payload Format for H.261 Video Streams & $4587 *$ \\
\hline-------- & $\begin{array}{l}\text { Extended RTP Profile for Real-time Transport Control } \\
\text { Protocol (RTCP)-Based Feedback (RTP/AVPF) }\end{array}$ & $4585 *$ \\
\hline-------- & $\begin{array}{l}\text { Session Description Protocol (SDP) Format for Binary } \\
\text { Floor Control Protocol (BFCP) Streams }\end{array}$ & $4583 *$ \\
\hline-------- & The Binary Floor Control Protocol (BFCP) & 4582 * \\
\hline-------- & $\begin{array}{l}\text { Cryptographically Generated Addresses (CGA) } \\
\text { Extension Field Format }\end{array}$ & 4581 * \\
\hline-------- & $\begin{array}{l}\text { Dynamic Host Configuration Protocol for IPv6 } \\
\text { (DHCPv6) Relay Agent Subscriber-ID option }\end{array}$ & $4580 *$ \\
\hline-------- & $\begin{array}{l}\text { OSPF as the Provider/Customer Edge Protocol for } \\
\text { BGP/MPLS IP Virtual Private Networks (VPNs) }\end{array}$ & 4577 * \\
\hline-------- & $\begin{array}{l}\text { Using a Link State Advertisement (LSA) Options Bit } \\
\text { to Prevent Looping in BGP/MPLS IP Virtual Private } \\
\text { Networks (VPNs) }\end{array}$ & $4576 *$ \\
\hline-------- & $\begin{array}{l}\text { A Session Initiation Protocol (SIP) Event Package } \\
\text { for Conference State }\end{array}$ & $4575 *$ \\
\hline-------- & $\begin{array}{l}\text { The Session Description Protocol (SDP) Label } \\
\text { Attribute }\end{array}$ & 4574 * \\
\hline-------- & $\begin{array}{l}\text { MIME Type Registration for RTP Payload Format for } \\
\text { H.224 }\end{array}$ & 4573 * \\
\hline -------- & $\begin{array}{l}\text { Connection-Oriented Media Transport over the } \\
\text { Transport Layer Security (TLS) Protocol in the } \\
\text { Session Description Protocol (SDP) }\end{array}$ & 4572 * \\
\hline --------- & $\begin{array}{l}\text { Framing Real-time Transport Protocol (RTP) and RTP } \\
\text { Control Protocol (RTCP) Packets over } \\
\text { Connection-Oriented Transport }\end{array}$ & 4571 * \\
\hline -------- & Session Description Protocol (SDP) Source Filters & 4570 * \\
\hline
\end{tabular}




\begin{tabular}{|c|c|c|}
\hline-------- & $\begin{array}{l}\text { Session Description Protocol (SDP) Security } \\
\text { Descriptions for Media Streams }\end{array}$ & 4568 * \\
\hline-------- & $\begin{array}{l}\text { Key Management Extensions for Session Description } \\
\text { Protocol (SDP) and Real Time Streaming Protocol } \\
\text { (RTSP) }\end{array}$ & $4567 *$ \\
\hline SDP & SDP : Session Description Protocol & $4566 *$ \\
\hline-------- & $\begin{array}{l}\text { The Key ID Information Type for the General } \\
\text { Extension Payload in Multimedia Internet KEYing } \\
\text { (MIKEY) }\end{array}$ & $4563 *$ \\
\hline-------- & $\begin{array}{l}\text { Definition of a Record Route Object (RRO) Node-Id } \\
\text { Sub-object }\end{array}$ & 4561 * \\
\hline-------- & $\begin{array}{l}\text { Definitions of Managed Objects for Remote Ping, } \\
\text { Traceroute, and Lookup Operations }\end{array}$ & $4560 *$ \\
\hline-------- & $\begin{array}{l}\text { Node-ID Based Resource Reservation Protocol (RSVP) } \\
\text { Hello: A Clarification Statement }\end{array}$ & 4558 * \\
\hline-------- & $\begin{array}{l}\text { Online Certificate Status Protocol (OCSP) Support } \\
\text { for Public Key Cryptography for Initial } \\
\text { Authentication in Kerberos (PKINIT) }\end{array}$ & $4557 *$ \\
\hline-------- & $\begin{array}{l}\text { Public Key Cryptography for Initial Authentication } \\
\text { in Kerberos (PKINIT) }\end{array}$ & 4556 * \\
\hline-------- & IKEv2 Mobility and Multihoming Protocol (MOBIKE) & $4555 *$ \\
\hline SATOP & $\begin{array}{l}\text { Structure-Agnostic Time Division Multiplexing (TDM) } \\
\text { over Packet (SAToP) }\end{array}$ & $4553 *$ \\
\hline-------- & Authentication/Confidentiality for OSPFv3 & 4552 * \\
\hline-------- & $\begin{array}{l}\text { IMAP Extension for Conditional STORE Operation or } \\
\text { Quick Flag Changes Resynchronization }\end{array}$ & 4551 * \\
\hline-------- & $\begin{array}{l}\text { Internet Email to Support Diverse Service } \\
\text { Environments (Lemonade) Profile }\end{array}$ & $4550 *$ \\
\hline-------- & $\begin{array}{l}\text { Internet Code Point (ICP) Assignments for NSAP } \\
\text { Addresses }\end{array}$ & $4548 *$ \\
\hline-------- & $\begin{array}{l}\text { Event Notification Management Information Base for } \\
\text { Data over Cable Service Interface Specifications } \\
\text { (DocSIS)-Compliant Cable Modems and Cable Modem } \\
\text { Termination Systems }\end{array}$ & $4547 *$ \\
\hline--- & $\begin{array}{l}\text { Radio Frequency ( } R F \text { ) Interface Management } \\
\text { Information Base for Data over Cable Service } \\
\text { Interface Specifications (DOCSIS) } 2.0 \text { Compliant RF } \\
\text { Interfaces }\end{array}$ & $4546 *$ \\
\hline-------- & $\begin{array}{l}\text { Definitions of Managed Objects for IP Storage User } \\
\text { Identity Authorization }\end{array}$ & $4545 *$ \\
\hline-------- & $\begin{array}{l}\text { Definitions of Managed Objects for Internet Small } \\
\text { Computer system Interface (iSCSI) }\end{array}$ & 4544 * \\
\hline-------- & $\begin{array}{l}\text { The Use of Galois Message Authentication Code (GMAC) } \\
\text { in IPsec ESP and AH }\end{array}$ & $4543 *$ \\
\hline-------- & $\begin{array}{l}\text { Request Authorization through Dialog Identification } \\
\text { in the Session Initiation Protocol (SIP) }\end{array}$ & 4538 * \\
\hline-------- & Kerberos Cryptosystem Negotiation Extension & \\
\hline
\end{tabular}




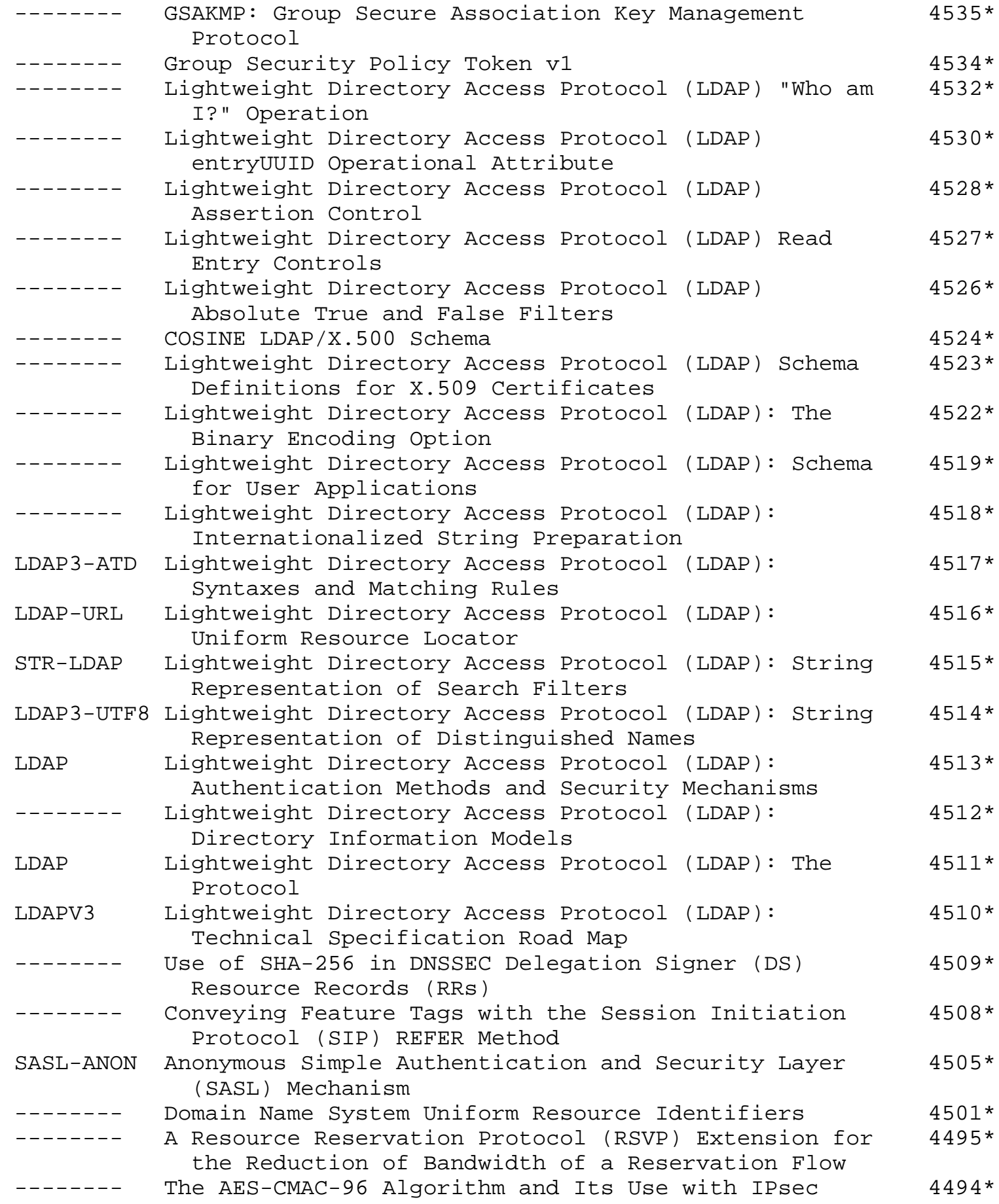




\begin{tabular}{|c|c|c|}
\hline & $\begin{array}{l}\text { Jsing the GOST R 34.10-94, GOST R 34.10-2001, and } \\
\text { GOST R 34.11-94 Algorithms with the Internet X.509 } \\
\text { Public Key Infrastructure Certificate and CRL Profile }\end{array}$ & \\
\hline-- & $\begin{array}{l}\text { Using the GOST 28147-89, GOST R 34.11-94, GOST R } \\
34.10-94 \text {, and GOST R 34.10-2001 Algorithms with } \\
\text { Cryptographic Message Syntax (CMS) }\end{array}$ & $4490 *$ \\
\hline-- & $\begin{array}{l}\text { A Method for Generating Link-Scoped IPv6 Multicast } \\
\text { Addresses }\end{array}$ & $4489 *$ \\
\hline -ー-ー-ー- & $\begin{array}{l}\text { Suppression of Session Initiation Protocol (SIP) } \\
\text { REFER Method Implicit Subscription }\end{array}$ & $4488 *$ \\
\hline------- & Subcodes for BGP Cease Notification Message & $4486 *$ \\
\hline---- & $\begin{array}{l}\text { A Mechanism for Content Indirection in Session } \\
\text { Initiation Protocol (SIP) Messages }\end{array}$ & \\
\hline ------- & $\begin{array}{l}\text { CIPID: Contact Information for the Presence } \\
\text { Information Data Format }\end{array}$ & $4482 *$ \\
\hline------- & $\begin{array}{l}\text { Timed Presence Extensions to the Presence } \\
\text { Information Data Format (PIDF) to Indicate Status } \\
\text { Information for Past and Future Time Intervals }\end{array}$ & 4481 * \\
\hline ------- & $\begin{array}{l}\text { RPID: Rich Presence Extensions to the Presence } \\
\text { Information Data Format (PIDF) }\end{array}$ & $4480 *$ \\
\hline------- & A Data Model for Presence & $79 *$ \\
\hline------- & Attribute Certificate ( $A C)$ Policies Extension & $76 *$ \\
\hline-------- & $\begin{array}{l}\text { Enhancements for Authenticated Identity Management } \\
\text { in the Session Initiation Protocol (SIP) }\end{array}$ & 4474 * \\
\hline------- & $\begin{array}{l}\text { Minimally Covering NSEC Records and DNSSEC On-line } \\
\text { Signing }\end{array}$ & $4470 *$ \\
\hline------- & $\begin{array}{l}\text { Internet Message Access Protocol (IMAP) CATENATE } \\
\text { Extension }\end{array}$ & $4469 *$ \\
\hline URLAUTH & Message Submission BURL Extension & 8 * \\
\hline-------- & $\begin{array}{l}\text { Internet Message Access Protocol (IMAP) - URLAUTH } \\
\text { Extension }\end{array}$ & $4467 *$ \\
\hline ----ー-- & Collected Extensions to IMAP 4 ABNF & $66 *$ \\
\hline------- & $\begin{array}{l}\text { Generic Security Service Application Program } \\
\text { Interface (GSS-API) Authentication and Key Exchange } \\
\text { for the Secure Shell (SSH) Protocol }\end{array}$ & 4462 * \\
\hline------- & $\begin{array}{l}\text { Definition of Managed Objects for Small Computer } \\
\text { System Interface (SCSI) Entities }\end{array}$ & $4455 *$ \\
\hline------- & $\begin{array}{l}\text { Asynchronous Transfer Mode (ATM) over Layer } 2 \\
\text { Tunneling Protocol Version } 3 \text { (L2TPV3) }\end{array}$ & 4454 * \\
\hline & $\begin{array}{l}\text { Securing Mobile IPv6 Route Optimization Using a } \\
\text { Static Shared Key }\end{array}$ & $4449 *$ \\
\hline & $\begin{array}{l}\text { Encapsulation Methods for Transport of Ethernet over } \\
\text { MPLS Networks }\end{array}$ & $4448 *$ \\
\hline-------- & $\begin{array}{l}\text { Pseudowire Setup and Maintenance Using the Label } \\
\text { Distribution Protocol (LDP) }\end{array}$ & 4447 * \\
\hline------- & Management Information Base for Intermediate system & \\
\hline
\end{tabular}




\begin{tabular}{|c|c|c|}
\hline-------- & $\begin{array}{l}\text { Bootstrapping Timed Efficient Stream Loss-Tolerant } \\
\text { Authentication (TESLA) }\end{array}$ & $4442 *$ \\
\hline-------- & Fibre Channel Fabric Address Manager MIB & $4439 *$ \\
\hline----- & Fibre-Channel Name Server MIB & $4438 *$ \\
\hline-------- & Detecting Network Attachment in IPv4 (DNAv4) & $4436 *$ \\
\hline-------- & $\begin{array}{l}\text { The AES-XCBC-PRF-128 Algorithm for the Internet Key } \\
\text { Exchange Protocol (IKE) }\end{array}$ & 4434 * \\
\hline-------- & Mobile IPv4 Dynamic Home Agent (HA) Assignment & $4433 *$ \\
\hline-------- & $\begin{array}{l}\text { RSA Key Exchange for the Secure Shell (SSH) } \\
\text { Transport Layer Protocol }\end{array}$ & 4432 * \\
\hline-------- & Kerberized Internet Negotiation of Keys (KINK) & 4430 * \\
\hline-------- & Optimistic Duplicate Address Detection (DAD) for IPv6 & $4429 *$ \\
\hline-------- & $\begin{array}{l}\text { Generalized Multi-Protocol Label Switching (GMPLS) } \\
\text { Recovery Functional Specification }\end{array}$ & $4426 *$ \\
\hline-------- & RTP Payload Format for Video Codec 1 (VC-1) & $4425 *$ \\
\hline-------- & $\begin{array}{l}\text { Real-Time Transport Protocol (RTP) Payload Format } \\
\text { for the Variable-Rate Multimode Wideband (VMR-WB) } \\
\text { Extension Audio Codec }\end{array}$ & 4424 * \\
\hline SASL & Simple Authentication and Security Layer (SASL) & $4422 *$ \\
\hline-------- & $\begin{array}{l}\text { RTP Payload Format for Uncompressed Video: } \\
\text { Additional Colour Sampling Modes }\end{array}$ & 4421 * \\
\hline-------- & $\begin{array}{l}\text { Encoding of Attributes for Multiprotocol Label } \\
\text { Switching (MPLS) Label Switched Path (LSP) } \\
\text { Establishment Using Resource ReserVation } \\
\text { Protocol-Traffic Engineering (RSVP-TE) }\end{array}$ & 4420 * \\
\hline-------- & $\begin{array}{l}\text { Diffie-Hellman Group Exchange for the Secure shell } \\
\text { (SSH) Transport Layer Protocol }\end{array}$ & 4419 * \\
\hline-------- & IANA Registration for Enumservice Voice & $4415 *$ \\
\hline-------- & $\begin{array}{l}\text { An ENUM Registry Type for the Internet Registry } \\
\text { Information Service (IRIS) }\end{array}$ & 4414 * \\
\hline-------- & $\begin{array}{l}\text { Communications Resource Priority for the Session } \\
\text { Initiation Protocol (SIP) }\end{array}$ & 4412 * \\
\hline-------- & $\begin{array}{l}\text { Extending the Session Initiation Protocol (SIP) } \\
\text { Reason Header for Preemption Events }\end{array}$ & 4411 * \\
\hline-------- & $\begin{array}{l}\text { Definitions of Managed Objects for Fibre Channel } \\
\text { Over TCP/IP (FCIP) }\end{array}$ & 4404 * \\
\hline-------- & $\begin{array}{l}\text { A Pseudo-Random Function (PRF) for the Kerberos V } \\
\text { Generic Security Service Application Program } \\
\text { Interface (GSS-API) Mechanism }\end{array}$ & 4402 * \\
\hline-------- & $\begin{array}{l}\text { A Pseudo-Random Function (PRF) API Extension for the } \\
\text { Generic Security Service Application Program } \\
\text { Interface (GSS-API) }\end{array}$ & 4401 * \\
\hline $\mathrm{SC}-\mathrm{DNS}$ & Storing Certificates in the Domain Name System (DNS) & 4398 * \\
\hline-------- & $\begin{array}{l}\text { RTP Payload Format for 3rd Generation Partnership } \\
\text { Project (3GPP) Timed Text }\end{array}$ & $4396 *$ \\
\hline-------- & MIME Type Registrations for 3GPP2 Multimedia Files & $4393 *$ \\
\hline-------- & Transmission of IP over InfiniBand (IPoIB) & $4391 *$ \\
\hline
\end{tabular}




\begin{tabular}{|c|c|c|}
\hline-------- & $\begin{array}{l}\text { Dynamic Host Configuration Protocol (DHCP) over } \\
\text { InfiniBand }\end{array}$ & 4390 * \\
\hline- & Dynamic Host Configuration Protocol (DHCP) Leasequery & 4388 * \\
\hline--- & $\begin{array}{l}\text { Internet X.509 Public Key Infrastructure Operational } \\
\text { Protocols: Certificate Store Access via HTTP }\end{array}$ & $387 \star$ \\
\hline----- & $\begin{array}{l}\text { Pseudowire Emulation Edge-to-Edge (PWE3) Control } \\
\text { Word for Use over an MPLS PSN }\end{array}$ & $4385 *$ \\
\hline---- & $\begin{array}{l}\text { The Use of Timed Efficient Stream Loss-Tolerant } \\
\text { Authentication (TESLA) in the Secure Real-time } \\
\text { Transport Protocol (SRTP) }\end{array}$ & $4383 *$ \\
\hline & $\begin{array}{l}\text { MPLS/BGP Layer } 3 \text { Virtual Private Network (VPN) } \\
\text { Management Information Base }\end{array}$ & 4382 * \\
\hline------ & $\begin{array}{l}\text { Teredo: Tunneling IPv6 over UDP through Network } \\
\text { Address Translations (NATs) }\end{array}$ & 4380 * \\
\hline-------- & $\begin{array}{l}\text { Detecting Multi-Protocol Label Switched (MPLS) Data } \\
\text { Plane Failures }\end{array}$ & $379 *$ \\
\hline-------- & Chargeable User Identity & $4372 *$ \\
\hline--- & $\begin{array}{l}\text { Lightweight Directory Access Protocol (LDAP) Proxied } \\
\text { Authorization Control }\end{array}$ & $4370 *$ \\
\hline------ & $\begin{array}{l}\text { Definitions of Managed Objects for Internet Fibre } \\
\text { Channel Protocol (iFCP) }\end{array}$ & $4369 *$ \\
\hline-------- & $\begin{array}{l}\text { Multiprotocol Label Switching (MPLS) } \\
\text { Label-Controlled Asynchronous Transfer Mode (ATM) } \\
\text { and Frame-Relay Management Interface Definition }\end{array}$ & 4368 * \\
\hline & Transport Layer Security (TLS) Extensions & $4366 *$ \\
\hline----- & BGP/MPLS IP Virtual Private Networks (VPNs) & 364 * \\
\hline----- & $\begin{array}{l}\text { Definitions of Managed Objects for Bridges with } \\
\text { Traffic Classes, Multicast Filtering, and Virtual } \\
\text { LAN Extensions }\end{array}$ & $4363 *$ \\
\hline--- & $\begin{array}{l}\text { RObust Header Compression }(\mathrm{ROHC}) \text { : A Link-Layer } \\
\text { Assisted Profile for IP/UDP/RTP }\end{array}$ & $4362 *$ \\
\hline-------- & $\begin{array}{l}\text { Node-specific Client Identifiers for Dynamic Host } \\
\text { Configuration Protocol Version Four (DHCPV4) }\end{array}$ & 4361 * \\
\hline----- & BGP Extended Communities Attribute & 4360 * \\
\hline-------- & $\begin{array}{l}\text { The Use of RSA/SHA-1 Signatures within Encapsulating } \\
\text { Security Payload (ESP) and Authentication Header ( } \mathrm{AH})\end{array}$ & $4359 *$ \\
\hline-------- & $\begin{array}{l}\text { Mapping Between the Multimedia Messaging Service } \\
\text { (MMS) and Internet Mail }\end{array}$ & $4356 *$ \\
\hline------ & $\begin{array}{l}\text { IANA Registration for Enumservices email, fax, mms, } \\
\text { ems, and sms }\end{array}$ & $4355 *$ \\
\hline-------- & $\begin{array}{l}\text { RTP Payload Format for the Extended Adaptive } \\
\text { Multi-Rate Wideband (AMR-WB+) Audio Codec }\end{array}$ & 4352 * \\
\hline-------- & $\begin{array}{l}\text { High-Level Data Link Control (HDLC) Frames over } \\
\text { Layer } 2 \text { Tunneling Protocol, Version } 3 \text { (L2TPv3) }\end{array}$ & $4349 *$ \\
\hline -------- & $\begin{array}{l}\text { Real-Time Transport Protocol (RTP) Payload Format } \\
\text { for the Variable-Rate Multimode Wideband (VMR-WB) } \\
\text { Audio Codec }\end{array}$ & $4348 *$ \\
\hline-------- & Datagram Transport Layer Security & 347 * \\
\hline
\end{tabular}




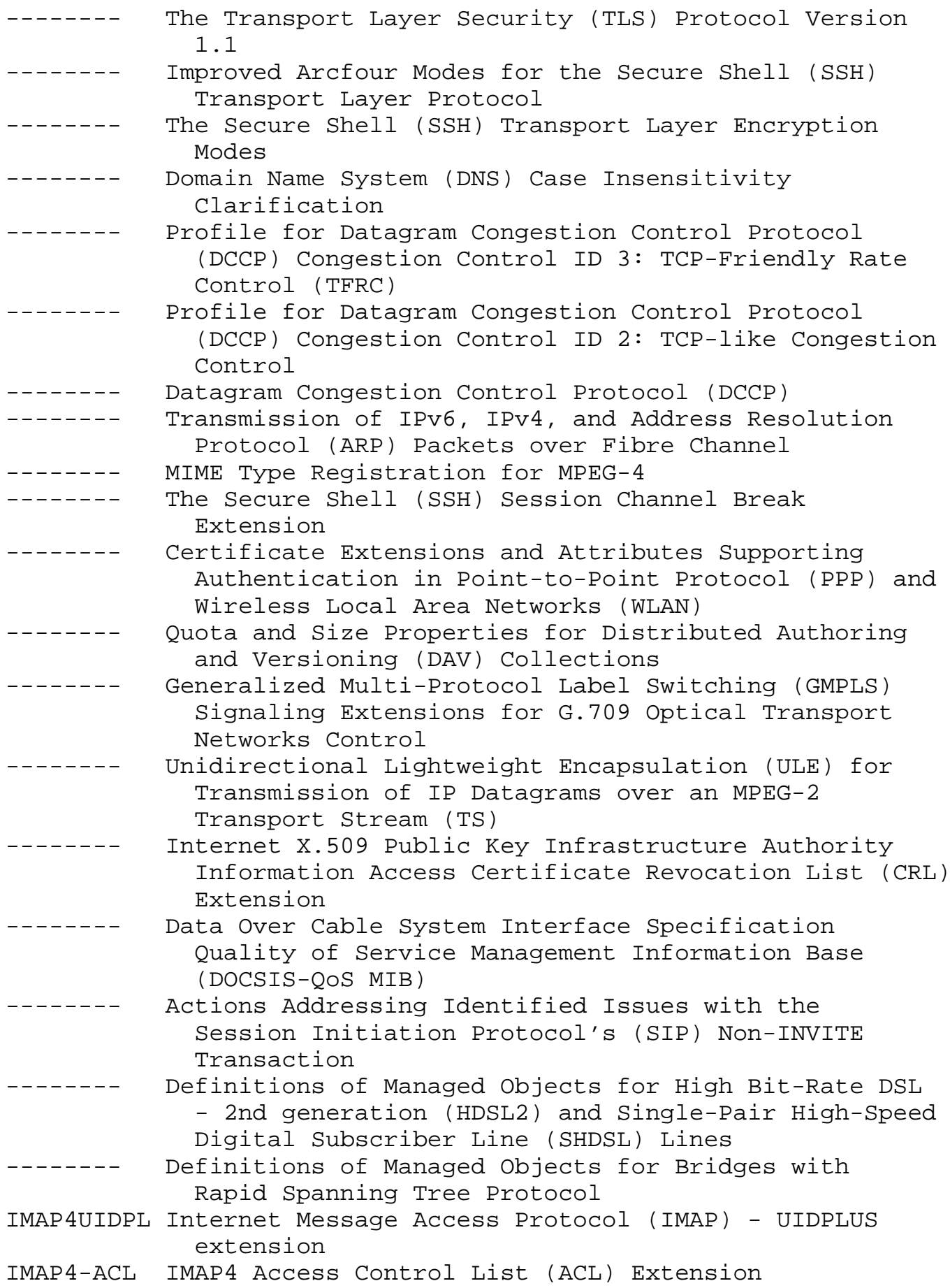




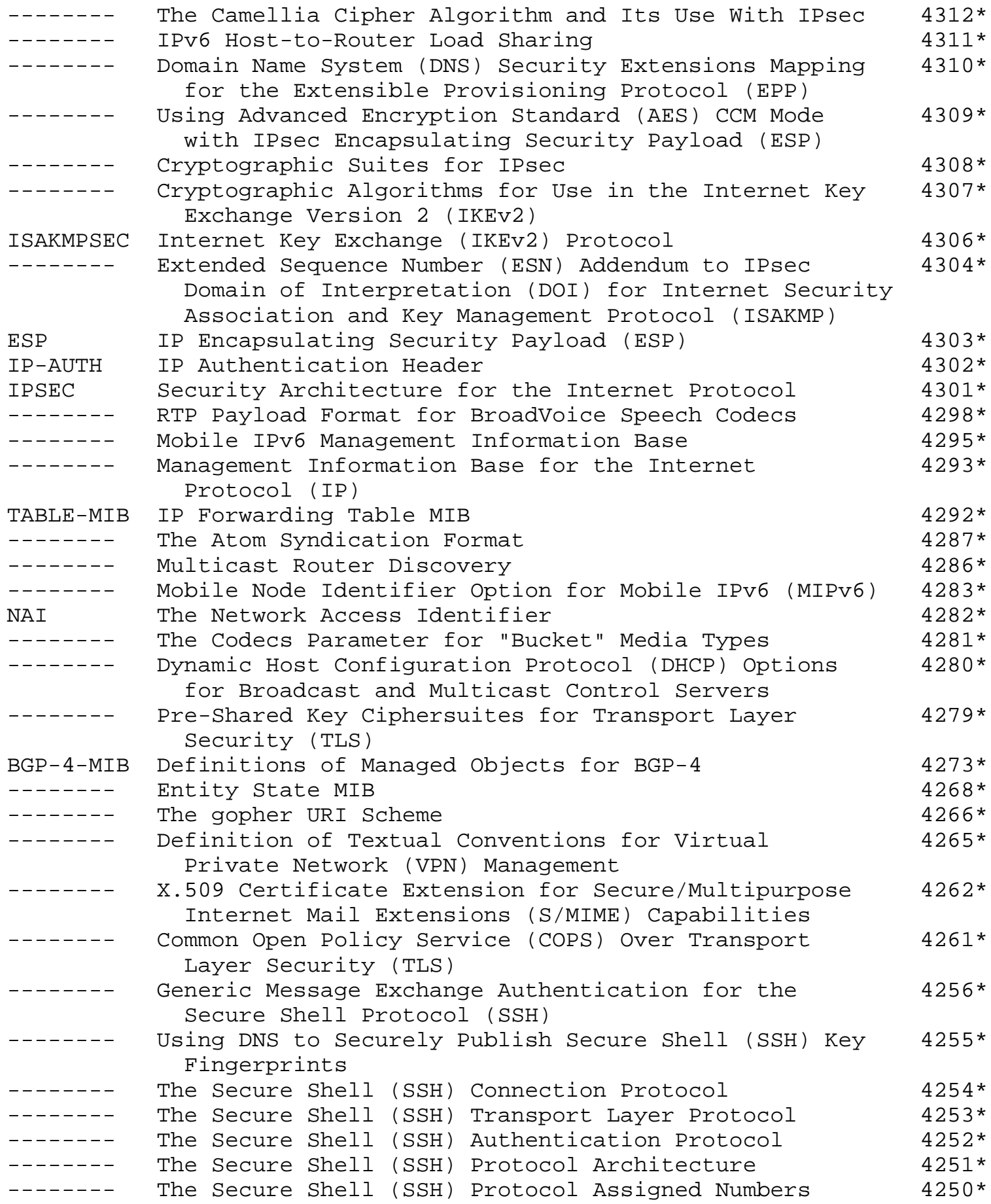




\begin{tabular}{|c|c|c|}
\hline--- & $\begin{array}{l}\text { The telnet URI Scheme } \\
\text { An Extension to the Session Initiation Protocol } \\
\text { (SIP) for Request History Information }\end{array}$ & 4244 * \\
\hline-- & $\begin{array}{l}\text { Vendor-Specific Information Suboption for the } \\
\text { Dynamic Host Configuration Protocol (DHCP) Relay } \\
\text { Agent Option }\end{array}$ & 43 * \\
\hline--- & $\begin{array}{l}\text { Information Refresh Time Option for Dynamic Host } \\
\text { Configuration Protocol for IPv6 (DHCPv6) }\end{array}$ & 4242 * \\
\hline------- & Internet Voice Messaging (IVM) & $39 *$ \\
\hline------- & Voice Message Routing Service & \\
\hline--- & Voice Messaging Directory Service & \\
\hline--- & $\begin{array}{l}\text { HTTP Adaptation with Open Pluggable Edge Services } \\
\text { (OPES) }\end{array}$ & \\
\hline------- & $\begin{array}{l}\text { An INVITE-Initiated Dialog Event Package for the } \\
\text { Session Initiation Protocol (SIP) }\end{array}$ & \\
\hline------- & $\begin{array}{l}\text { Integrated Services Digital Network (ISDN) } \\
\text { Q.921-User Adaptation Layer }\end{array}$ & \\
\hline------- & $\begin{array}{l}\text { Identifiers and Test Vectors for HMAC-SHA-224, } \\
\text { HMAC-SHA-256, HMAC-SHA-384, and HMAC-SHA-512 }\end{array}$ & \\
\hline--- & $\begin{array}{l}\text { Using the Simple Object Access Protocol (SOAP) in } \\
\text { Blocks Extensible Exchange Protocol (BEEP) }\end{array}$ & \\
\hline------- & Traffic Engineering Link Management Information Base & \\
\hline-------- & Securing FTP with TLS & \\
\hline TRANS-IPV6 & $\begin{array}{l}\text { Basic Transition Mechanisms for IPv6 Hosts and } \\
\text { Routers }\end{array}$ & \\
\hline . 509-CRMF & $\begin{array}{l}\text { Internet X.509 Public Key Infrastructure Certificate } \\
\text { Request Message Format (CRMF) }\end{array}$ & \\
\hline PKICMP & $\begin{array}{l}\text { Internet X.509 Public Key Infrastructure Certificate } \\
\text { Management Protocol (CMP) }\end{array}$ & \\
\hline---- & $\begin{array}{c}\text { Link Management Protocol (LMP) for Dense Wavelength } \\
\text { Division Multiplexing (DWDM) Optical Line Systems }\end{array}$ & \\
\hline & $\begin{array}{l}\text { Generalized Multiprotocol Label Switching (GMPLS) } \\
\text { User-Network Interface (UNI): Resource ReserVation } \\
\text { Protocol-Traffic Engineering (RSVP-TE) Support for } \\
\text { the Overlay Model }\end{array}$ & \\
\hline----- & $\begin{array}{l}\text { Synchronous Optical Network (SONET)/Synchronous } \\
\text { Digital Hierarchy (SDH) Encoding for Link Management } \\
\text { Protocol (LMP) Test Messages }\end{array}$ & \\
\hline & $\begin{array}{l}\text { Label Switched Paths (LSP) Hierarchy with } \\
\text { Generalized Multi-Protocol Label Switching (GMPLS) } \\
\text { Traffic Engineering (TE) }\end{array}$ & $06 *$ \\
\hline & Link Management Protocol (LMP) & \\
\hline-------- & $\begin{array}{l}\text { OSPF Extensions in Support of Generalized } \\
\text { Multi-Protocol Label Switching (GMPLS) }\end{array}$ & \\
\hline -------- & $\begin{array}{l}\text { Routing Extensions in Support of Generalized } \\
\text { Multi-Protocol Label Switching (GMPLS) }\end{array}$ & 4202 * \\
\hline & Link Bundling in MPLS Traffic Engineering (TE) & \\
\hline-------- & The SEED Cipher Algorithm and Its Use with IPsec & \\
\hline
\end{tabular}




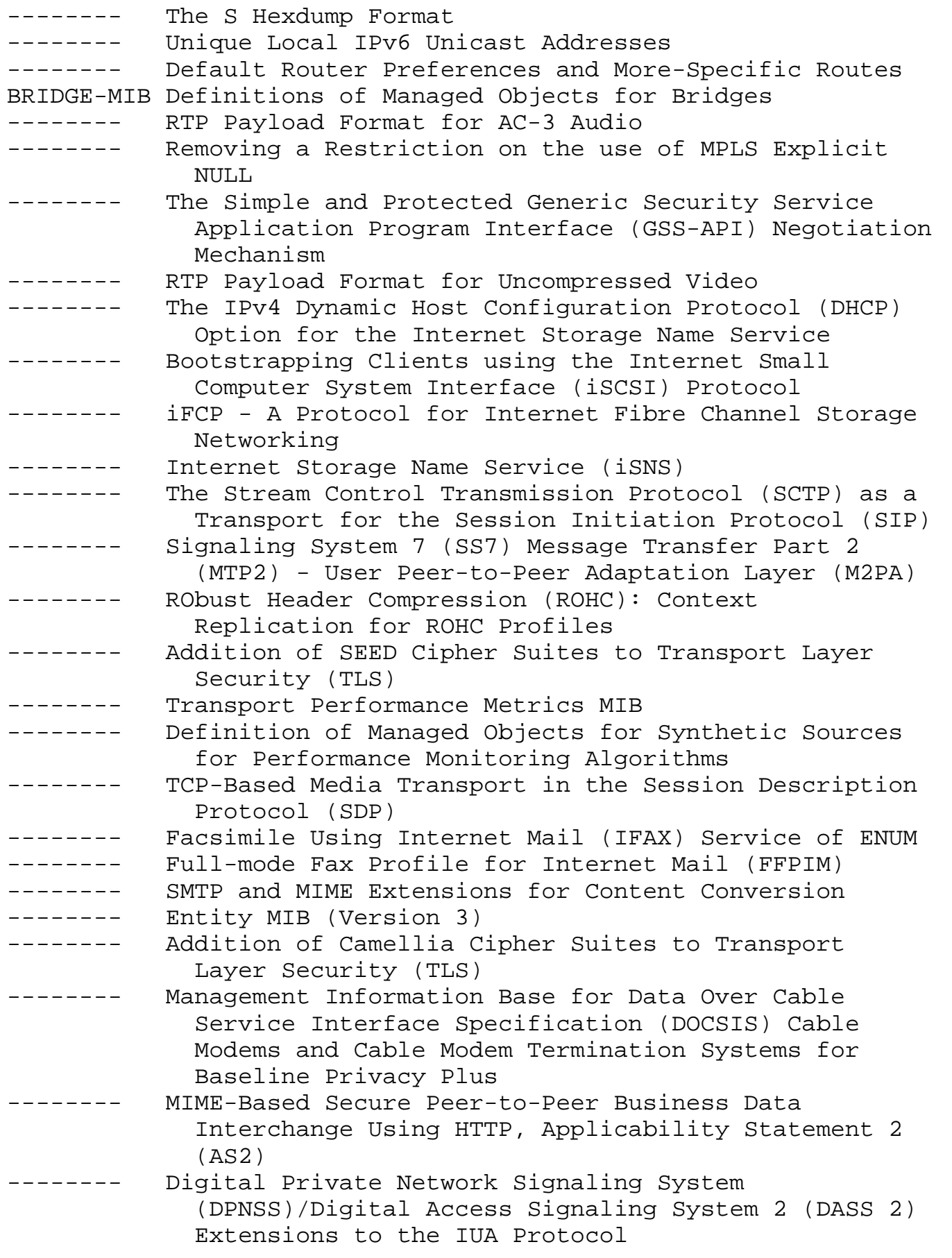

$4194 *$

4193*

$4191 *$

$4188 *$

4184 *

4182 *

4178 *

4175 *

4174 *

4173*

4172 *

4171 *

$4168 *$

$4165 *$

4164 *

4162 *

4150 *

4149*

$4145 *$

4143 *

4142 *

4141 *

$4133 *$

4132 *

4131 *

4130 *

4129* 


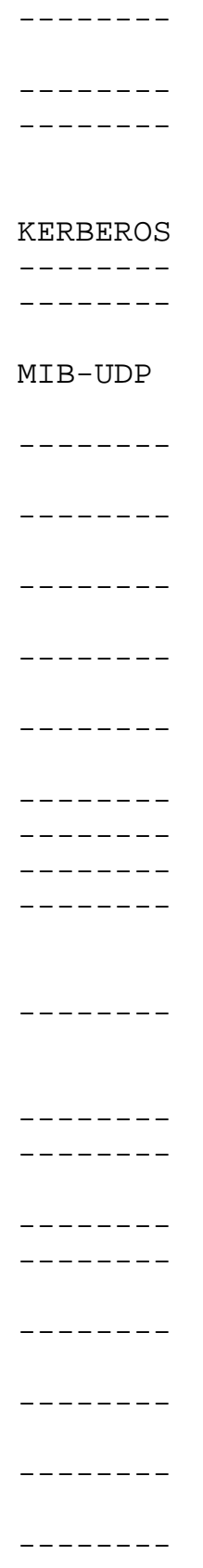

Protocol Extensions for Support of Diffserv-aware MPLS Traffic Engineering

A Universally Unique IDentifier (UUID) URN Namespace 4122*

The Kerberos Version 5 Generic Security Service 4121* Application Program Interface (GSS-API) Mechanism: Version 2

The Kerberos Network Authentication Service (V5) 4120*

A Presence-based GEOPRIV Location Object Format 4119*

E.164 Number Mapping for the Extensible Provisioning 4114 * Protocol (EPP)

Management Information Base for the User Datagram 4113* Protocol (UDP)

Electronic Commerce Modeling Language (ECML) Version 4112* 2 Specification

Algorithms for Internet Key Exchange version 1 4109* ( IKEv1)

Using Cryptographic Message Syntax (CMS) to Protect 4108* Firmware Packages

The Use of Galois/Counter Mode (GCM) in IPsec Encapsulating Security Payload (ESP)

Policy Core Extension Lightweight Directory Access 4104 * Protocol Schema (PCELS)

RTP Payload for Text Conversation 4103*

Registration of the text/red MIME Sub-Type 4102*

Attaching Meaning to Solicitation Class Keywords 4095*

Usage of the Session Description Protocol (SDP) 4092* Alternative Network Address Types (ANAT) Semantics in the Session Initiation Protocol (SIP)

The Alternative Network Address Types (ANAT) 4091* Semantics for the Session Description Protocol (SDP) Grouping Framework

Fast Reroute Extensions to RSVP-TE for LSP Tunnels 4090*

Uniform Resource Identifier (URI) Scheme for the 4088* Simple Network Management Protocol (SNMP)

IP Tunnel MIB

A Negative Acknowledgement Mechanism for Signaling Compression

Simple Network Time Protocol (SNTP) Configuration 4075* Option for DHCPv6

Protecting Multiple Contents with the Cryptographic 4073* Message Syntax (CMS)

Diameter Extensible Authentication Protocol (EAP) 4072* Application

Definitions of Managed Object Extensions for Very 4070* High speed Digital Subscriber Lines (VDSL) Using Multiple Carrier Modulation (MCM) Line Coding 


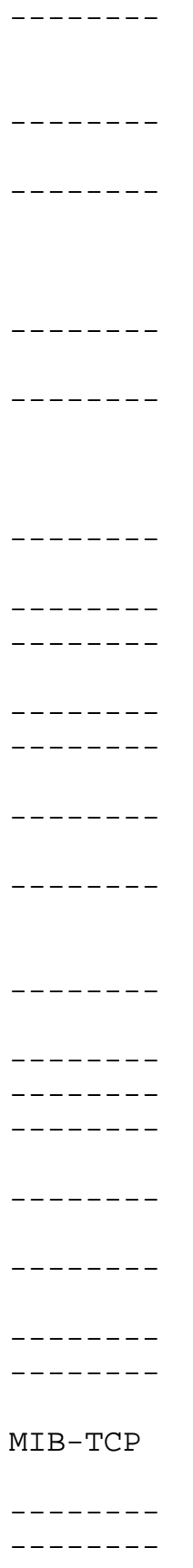

Definitions of Managed Object Extensions for Very High Speed Digital Subscriber Lines (VDSL) Using Single Carrier Modulation (SCM) Line Coding

Experimental Message, Extensions, and Error Codes for Mobile IPv4

RTP Payload Formats for European Telecommunications Standards Institute (ETSI) European Standard ES 202 050, ES 202 211, and ES 202212 Distributed Speech Recognition Encoding

Use of the RSASSA-PSS Signature Algorithm in Cryptographic Message Syntax (CMS)

Additional Algorithms and Identifiers for RSA Cryptography for use in the Internet X.509 Public Key Infrastructure Certificate and Certificate Revocation List (CRL) Profile

Additional XML Security Uniform Resource Identifiers (URIs)

Fibre Channel Management MIB

Internet X.509 Public Key Infrastructure Permanent Identifier

RTP Payload Format for a $64 \mathrm{kbit} / \mathrm{s}$ Transparent Call 4040*

Rapid Commit Option for the Dynamic Host Configuration Protocol version 4 (DHCPV4)

Open Pluggable Edge Services (OPES) Callout Protocol 4037* (OCP) Core

Management Information Base for Data Over Cable Service Interface Specification (DOCSIS) Cable Modem Termination Systems for Subscriber Management

Protocol Modifications for the DNS Security Extensions

Resource Records for the DNS Security Extensions

DNS Security Introduction and Requirements

Update to the Session Initiation Protocol (SIP) Preconditions Framework

The Authentication Suboption for the Dynamic Host Configuration Protocol (DHCP) Relay Agent Option

Session Timers in the Session Initiation Protocol (SIP)

A Method for Storing IPsec Keying Material in DNS

Encapsulating MPLS in IP or Generic Routing Encapsulation (GRE)

Management Information Base for the Transmission Control Protocol (TCP)

Registration of Mail and MIME Header Fields 4021* Robust Header Compression (ROHC): Profiles for User Datagram Protocol (UDP) Lite

4064 *

4060 *

$4056 *$

4055 *

4051 *

4044 *

$4043^{*}$

$4039 *$

4036 *

4035 *

4034 *

4033 *

4032 *

4030 *

4028 *

4025 *

$4023 *$

4022 *

4019* 


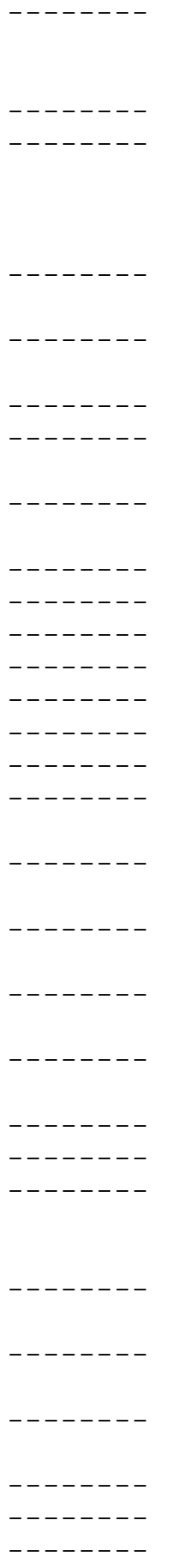

Finding Internet Small Computer Systems Interface $4018 *$ (iSCSI) Targets and Name Servers by Using Service Location Protocol version 2 (SLPV2)

The Eifel Response Algorithm for TCP

Remote Authentication Dial-In User Service (RADIUS) Attributes Suboption for the Dynamic Host Configuration Protocol (DHCP) Relay Agent Information Option

SASLprep: Stringprep Profile for User Names and 4013* Passwords

Routing Policy Specification Language next 4012* generation (RPSLng)

Policy Based Management MIB

Use of the SEED Encryption Algorithm in Cryptographic Message Syntax (CMS)

Definitions of Managed objects for Network Address 4008* Translators (NAT)

IPv6 Scoped Address Architecture

Diameter Credit-Control Application

Diameter Network Access Server Application

Diameter Mobile IPv4 Application

GMPLS Signaling Procedure for Egress Control

IANA Registration for Enumservice 'web' and 'ft'

Textual Conventions for Internet Network Addresses

Internet Printing Protocol (IPP): Job and Printer Administrative Operations

Internet Printing Protocol (IPP): The 'ippget' Delivery Method for Event Notifications

Internet Printing Protocol (IPP): Event Notifications and Subscriptions

Indication of Message Composition for Instant 3994* Messaging

Subscriber-ID Suboption for the Dynamic Host 3993* Configuration Protocol (DHCP) Relay Agent Option

Internationalized Resource Identifiers (IRIs) 3987*

RTP Payload Format for H.264 Video 3984*

Using the Internet Registry Information Service 3983* (IRIS) over the Blocks Extensible Exchange Protocol (BEEP)

IRIS: A Domain Registry (dreg) Type for the

3982 *

Internet Registry Information Service (IRIS)

IRIS: The Internet Registry Information Service (IRIS) Core Protocol

T11 Network Address Authority (NAA) Naming Format for iSCSI Node Names

Network News Transfer Protocol (NNTP)

Cryptographically Generated Addresses (CGA)

$4007 *$

$4006 *$

$4005 *$

$4004^{*}$

$4003 *$

4002 *

4001 *

$3998 *$

3996*

3995*

SEcure Neighbor Discovery (SEND)

3981 *

3980 *

3977 *

3972*

$3971 *$

RFC Editor

Informational

[Page 33] 


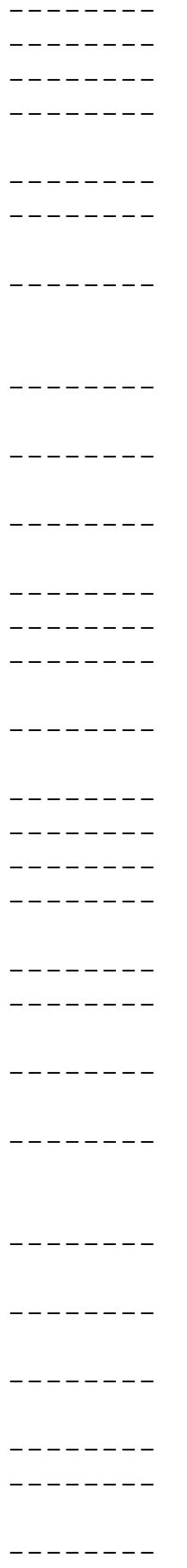

A Traffic Engineering (TE) MIB 


\begin{tabular}{|c|c|c|}
\hline-------- & $\begin{array}{l}\text { Session Initiation Protocol (SIP) Extension for } \\
\text { Event State Publication }\end{array}$ & $3903 *$ \\
\hline --------- & Network Information Service (NIS) Configuration & $3898 *$ \\
\hline & $\begin{array}{l}\text { Options for Dynamic Host Configuration Protocol for } \\
\text { IPv6 (DHCPv6) }\end{array}$ & \\
\hline DS3-E3-MIB & $\begin{array}{l}\text { Definitions of Managed Objects for the DS3/E3 } \\
\text { Interface Type }\end{array}$ & $3896 *$ \\
\hline-------- & Sieve Extension: Copying Without Side Effects & 3894 * \\
\hline-------- & $\begin{array}{l}\text { Session Initiation Protocol (SIP) Authenticated } \\
\text { Identity Body (AIB) Format }\end{array}$ & $3893 *$ \\
\hline-------- & $\begin{array}{l}\text { The Session Initiation Protocol (SIP) Referred-By } \\
\text { Mechanism }\end{array}$ & $3892 *$ \\
\hline-------- & $\begin{array}{l}\text { The Session Initiation Protocol (SIP) "Replaces" } \\
\text { Header }\end{array}$ & 3891 * \\
\hline-------- & $\begin{array}{l}\text { A Transport Independent Bandwidth Modifier for the } \\
\text { Session Description Protocol (SDP) }\end{array}$ & $3890 *$ \\
\hline-------- & Message Tracking Query Protocol & $3887 *$ \\
\hline----- & $\begin{array}{l}\text { An Extensible Message Format for Message Tracking } \\
\text { Responses }\end{array}$ & $3886 *$ \\
\hline-------- & SMTP Service Extension for Message Tracking & $3885 *$ \\
\hline-------- & $\begin{array}{l}\text { Detecting Inactive Neighbors over OSPF Demand } \\
\text { Circuits (DC) }\end{array}$ & $3883 *$ \\
\hline-------- & $\begin{array}{l}\text { Call Processing Language (CPL): A Language for User } \\
\text { Control of Internet Telephony Services }\end{array}$ & $3880 *$ \\
\hline--- & Deprecating Site Local Addresses & $3879 *$ \\
\hline---- & $\begin{array}{l}\text { Alarm Reporting Control Management Information Base } \\
\text { (MIB) }\end{array}$ & $3878 *$ \\
\hline-------- & Alarm Management Information Base (MIB) & $3877 *$ \\
\hline-------- & $\begin{array}{l}\text { Returning Matched Values with the Lightweight } \\
\text { Directory Access Protocol version } 3 \text { (LDAPv3) }\end{array}$ & $3876 *$ \\
\hline---- & $\begin{array}{l}\text { Stream Control Transmission Protocol (SCTP) } \\
\text { Management Information Base (MIB) }\end{array}$ & $3873 *$ \\
\hline-------- & $\begin{array}{l}\text { Management Information Base for Telephony Routing } \\
\text { over IP (TRIP) }\end{array}$ & 3872 * \\
\hline-------- & $\begin{array}{l}\text { Signalling Connection Control Part User Adaptation } \\
\text { Layer (SUA) }\end{array}$ & $3868 *$ \\
\hline-------- & $\begin{array}{l}\text { Language Tags and Ranges in the Lightweight } \\
\text { Directory Access Protocol (LDAP) }\end{array}$ & 3866 \\
\hline-------- & $\begin{array}{l}\text { A No Soliciting Simple Mail Transfer Protocol (SMTP) } \\
\text { Service Extension }\end{array}$ & $3865 *$ \\
\hline-------- & Presence Information Data Format (PIDF) & $3863 *$ \\
\hline----- & $\begin{array}{l}\text { Common Presence and Instant Messaging (CPIM): } \\
\text { Message Format }\end{array}$ & 3862 * \\
\hline-------- & Address Resolution for Instant Messaging and Presence & $3861 *$ \\
\hline-------- & Common Profile for Instant Messaging (CPIM) & $3860 *$ \\
\hline-------- & Common Profile for Presence (CPP) & $3859 *$ \\
\hline-------- & $\begin{array}{l}\text { An Extensible Markup Language (XML) Based Format for } \\
\text { Watcher Information }\end{array}$ & 3858 * \\
\hline
\end{tabular}




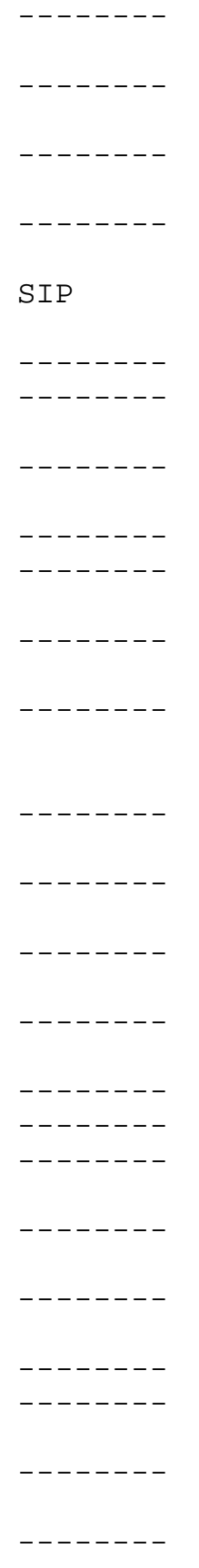

A Watcher Information Event Template-Package for the Session Initiation Protocol (SIP)

A Presence Event Package for the Session Initiation 3856* Protocol (SIP)

Transporting Secure/Multipurpose Internet Mail Extensions (S/MIME) Objects in X.400

Securing X.400 Content with Secure/Multipurpose Internet Mail Extensions (S/MIME)

S/MIME Advanced Encryption Standard (AES)

Requirement for the Session Initiation Protocol (SIP)

Cryptographic Message Syntax (CMS)

3853

3852

ecure/Multipurpose Internet Mail Extensions

3851

(S/MIME) Version 3.1 Message Specification

Secure/Multipurpose Internet Mail Extensions (S/MIME) Version 3.1 Certificate Handling

ESMTP and LMTP Transmission Types Registration

3850

Mobile IPv4 Extension for Carrying Network Access Identifiers

Robust Header Compression (ROHC): A Compression Profile for IP

A Message Summary and Message Waiting Indication Event Package for the Session Initiation Protocol (SIP)

Caller Preferences for the Session Initiation Protocol (SIP)

Indicating User Agent Capabilities in the Session Initiation Protocol (SIP)

MIME Type Registrations for 3rd Generation Partnership Project (3GPP) Multimedia files

Recommendations for Automatic Responses to Electronic Mail

MIKEY: Multimedia Internet KEYing

The Lightweight User Datagram Protocol (UDP-Lite)

The Advanced Encryption Standard (AES) Cipher Algorithm in the SNMP User-based Security Model

Dynamic Host Configuration Protocol option for Coordinate-based Location Configuration Information

Finding Fibre Channel over TCP/IP (FCIP) Entities Using Service Location Protocol version 2 (SLPv2)

Fibre Channel Over TCP/IP (FCIP)

3848

3846

3843

3842 *

Internet X.509 Public Key Infrastructure (PKI) Proxy Certificate Profile

Definitions of Managed Objects for Robust Header Compression ( $\mathrm{ROHC}$ )

Definitions of Managed Objects for the Multiprotocol Label Switching (MPLS), Label Distribution Protocol (LDP) 


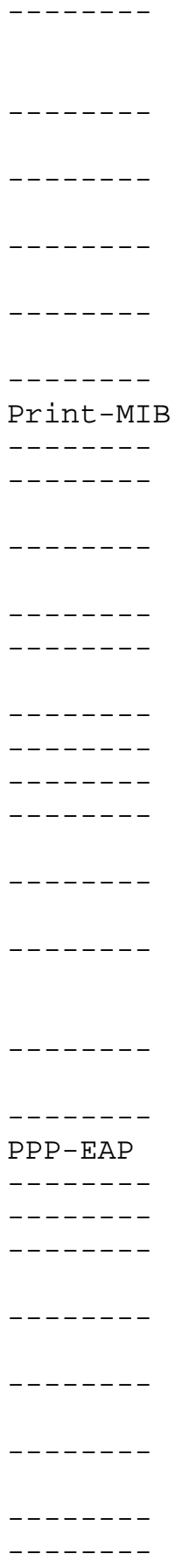

Multiprotocol Label Switching (MPLS) Forwarding

3814 Equivalence Class To Next Hop Label Forwarding Entry (FEC-To-NHLFE) Management Information Base (MIB)

Multiprotocol Label Switching (MPLS) Label Switching 3813 Router (LSR) Management Information Base (MIB)

Multiprotocol Label Switching (MPLS) Traffic Engineering (TE) Management Information Base (MIB)

Definitions of Textual Conventions (TCs) for Multiprotocol Label Switching (MPLS) Management

Multicast Listener Discovery Version 2 (MLDv2) for IPV 6

V5.2-User Adaptation Layer (V5UA) 3807

Printer MIB v2 3805

Voice Profile for Internet Mail (VPIM) Addressing 3804

Security Considerations for Signaling Transport 3788 (SIGTRAN) Protocols

The NewReno Modification to TCP's Fast Recovery Algorithm

X.509 Extensions for IP Addresses and AS Identifiers 3779

Using IPsec to Protect Mobile IPv6 Signaling Between 3776 Mobile Nodes and Home Agents

Mobility Support in IPv6

int-to-Point Protocol (PPP) Vendor Protocol

Securely Available Credentials Protocol $\quad 3767$

enumservice registration for Session Initiation 3764

Protocol (SIP) Addresses-of-Record

Telephone Number Mapping (ENUM) Service Registration 3762 for H. 323

The E.164 to Uniform Resource Identifiers (URI) 3761

Dynamic Delegation Discovery System (DDDS)

Application (ENUM)

Stream Control Transmission Protocol (SCTP) Partial Reliability Extension

Transport Layer Security Protocol Compression Methods 3749

Extensible Authentication Protocol (EAP) 3748

The Differentiated Services Configuration MIB 3747

MIME Type Registrations for JPEG 2000 (ISO/IEC 15444) 3745

Web Distributed Authoring and Versioning (WebDAV) 3744 Access Control Protocol

Internet X.509 Public Key Infrastructure: Qualified 3739 Certificates Profile

IANA Guidelines for the Registry of Remote 3737 Monitoring (RMON) MIB modules

Stateless Dynamic Host Configuration Protocol (DHCP) 3736 Service for IPv6

Application Performance Measurement MIB 3729

Definitions of Managed Objects for Very High speed 3728 Digital Subscriber Lines (VDSL) 


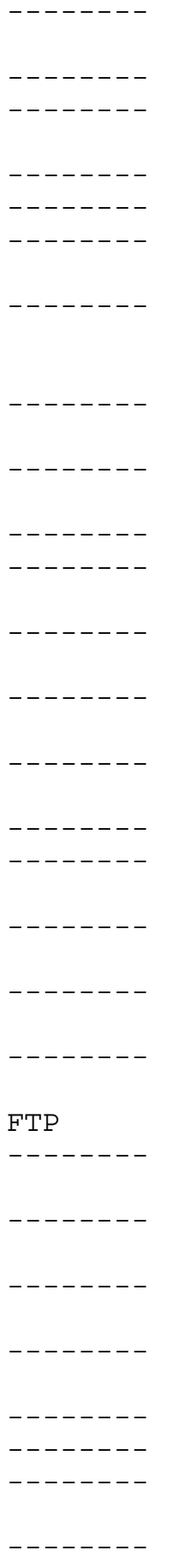

ASN.1 Module Definition for the LDAP and X.500 Component Matching Rules

Securing Block Storage Protocols over IP

String Profile for Internet Small Computer systems Interface (iSCSI) Names

Internet Small Computer Systems Interface (iSCSI) 3720

The Secure Real-time Transport Protocol (SRTP) 3711

Internet X.509 Public Key Infrastructure: Logotypes 3709 in X.509 Certificates

High Capacity Textual Conventions for MIB Modules Using Performance History Based on 15 Minute Intervals

Policy Core Lightweight Directory Access Protocol (LDAP) Schema

Lightweight Directory Access Protocol (LDAP): Additional Matching Rules

IPv6 Flow Label Specification

Internet Message Access Protocol (IMAP) UNSELECT command

Lightweight Directory Access Protocol (LDAP) and X.500 Component Matching Rules

Using Advanced Encryption Standard (AES) Counter 3686 Mode With IPsec Encapsulating Security Payload (ESP)

A Session Initiation Protocol (SIP) Event Package for Registrations

The Text/Plain Format and Delsp Parameters

3703

Lightweight Directory Access Protocol version 3 (LDAPV3) : All Operational Attributes

Subentries in the Lightweight Directory Access Protocol (LDAP)

Collective Attributes in the Lightweight Directory Access Protocol (LDAP)

Information Model for Describing Network Device QoS Datapath Mechanisms

Extensions to FTP

3698

Use of the Camellia Encryption Algorithm in Cryptographic Message Syntax (CMS)

Web Distributed Authoring and Versioning (WebDAV) Ordered Collections Protocol

DNS Configuration options for Dynamic Host Configuration Protocol for IPv6 (DHCPv6)

Generic Security Service Algorithm for Secret Key Transaction Authentication for DNS (GSS-TSIG)

Policy Quality of Service (QOS) Information Model 3644

Fibre Channel (FC) Frame Encapsulation 3643

Common Elements of Generic String Encoding Rules 3642 (GSER) Encodings

Generic String Encoding Rules (GSER) for ASN.1 Types 3641 


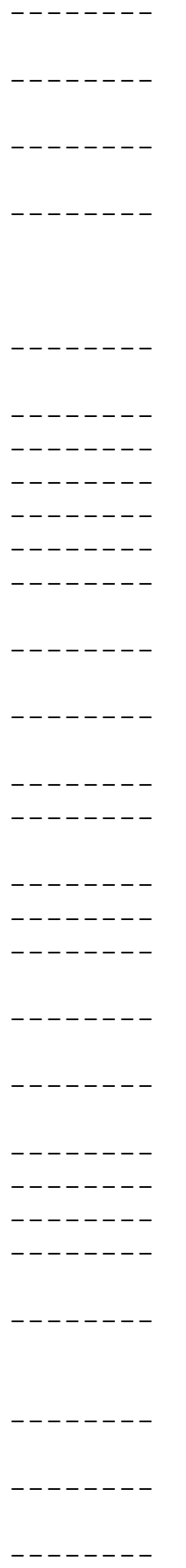

RTP Payload Format for Transport of MPEG-4 Elementary Streams

Definitions of Managed Objects for the Ethernet WAN 3637 Interface Sublayer

Definitions of Managed Objects for the Ethernet-like 3635 Interface Types

Key Distribution Center (KDC) Server Address Sub-option for the Dynamic Host Configuration Protocol (DHCP) CableLabs Client Configuration (CCC) Option

IPv6 Prefix Options for Dynamic Host Configuration Protocol (DHCP) version 6

Traffic Engineering (TE) Extensions to OSPF Version 23630

Graceful OSPF Restart 3623

Power Ethernet MIB $\quad 3621$

The TUNNEL Profile $\quad 3620$

RTP Control Protocol Extended Reports (RTCP XR) 3611

Session Initiation Protocol (SIP) Extension Header 3608

Field for Service Route Discovery During Registration

Definitions of Supplemental Managed Objects for ATM 3606 Interface

Real Time Control Protocol (RTCP) attribute in 3605 Session Description Protocol (SDP)

The AES-CBC Cipher Algorithm and Its Use with IPsec 3602

Text String Notation for Dial Sequences and Global 3601 Switched Telephone Network (GSTN) / E.164 Addresses

Handling of Unknown DNS Resource Record (RR) Types 3597

Textual Conventions for IPv6 Flow Label 3595

PacketCable Security Ticket Control Sub-Option for 3594 the DHCP CableLabs Client Configuration (CCC) Option

Definitions of Managed Objects for the Optical 3591 Interface Type

Source Address Selection for the Multicast Listener 3590 Discovery (MLD) Protocol

Diameter Base Protocol 3588

IP Security Policy (IPSP) Requirements 3586

IPsec Configuration Policy Information Model 3585

An Extension to the Session Initiation Protocol 3581 (SIP) for Symmetric Response Routing

Mapping of Integrated Services Digital Network 3578 (ISDN) User Part (ISUP) Overlap Signalling to the Session Initiation Protocol (SIP)

IANA Considerations for RADIUS (Remote Authentication Dial In User Service)

Signalling of Modem-On-Hold status in Layer 2 Tunneling Protocol (L2TP)

The AES-XCBC-MAC-96 Algorithm and Its Use With IPsec 3566 


\begin{tabular}{|c|c|c|}
\hline & $\begin{array}{l}\text { Encryption Algorithm in Cryptographic Message Syntax } \\
\text { (CMS) }\end{array}$ & \\
\hline-- & $\begin{array}{l}\text { Use of the RSAES-OAEP Key Transport Algorithm in } \\
\text { Cryptographic Message Syntax (CMS) }\end{array}$ & 3560 \\
\hline-- & Multicast Address Allocation MIB & 3559 \\
\hline-- & $\begin{array}{l}\text { RTP Payload Format for Enhanced Variable Rate Codecs } \\
\text { (EVRC) and Selectable Mode Vocoders (SMV) }\end{array}$ & \\
\hline-------- & RTP Payload Format for European Telecommunications & 35 \\
\hline & $\begin{array}{l}\text { Standards Institute (ETSI) European Standard ES } 201 \\
108 \text { Distributed Speech Recognition Encoding }\end{array}$ & \\
\hline-------- & Session Description Protocol (SDP) Bandwidth & 6 \\
\hline & Modifiers for RTP Control Protocol (RTCP) Bandwidth & \\
\hline-------- & $\begin{array}{l}\text { On the Use of Stream Control Transmission Protocol } \\
\text { (SCTP) with IPsec }\end{array}$ & \\
\hline ----ー--- & The Group Domain of Interpretation & \\
\hline--- & $\begin{array}{l}\text { Enhanced Compressed RTP (CRTP) for Links with High } \\
\text { Delay, Packet Loss and Reordering }\end{array}$ & \\
\hline IPCOM-PPP & IP Header Compression over PPP & \\
\hline--- & Registration Revocation in Mobile IPv4 & \\
\hline-------- & $\begin{array}{l}\text { Authentication, Authorization and Accounting (AAA) } \\
\text { Transport Profile }\end{array}$ & \\
\hline-- & Wrapping a Hashed Message Authentication Code (HMAC) & \\
\hline & $\begin{array}{l}\text { key with a Triple-Data Encryption Standard (DES) Key } \\
\text { or an Advanced Encryption Standard (AES) Key }\end{array}$ & \\
\hline-------- & The application/ogg Media Type & \\
\hline NFSv 4 & Network File System (NFS) version 4 Protocol & \\
\hline-------- & $\begin{array}{l}\text { Link Selection sub-option for the Relay Agent } \\
\text { Information Option for DHCPv } 4\end{array}$ & \\
\hline-------- & $\begin{array}{l}\text { More Modular Exponential (MODP) Diffie-Hellman } \\
\text { groups for Internet Key Exchange (IKE) }\end{array}$ & \\
\hline-------- & $\begin{array}{l}\text { Mapping of Media Streams to Resource Reservation } \\
\text { Flows }\end{array}$ & 4 \\
\hline --ー-ー--- & Session Authorization Policy Element & \\
\hline-------- & $\begin{array}{l}\text { Mobile IP Traversal of Network Address Translation } \\
\text { (NAT) Devices }\end{array}$ & \\
\hline $\mathrm{PPP}-\mathrm{BCP}$ & $\begin{array}{l}\text { Point-to-Point Protocol (PPP) Bridging Control } \\
\text { Protocol (BCP) }\end{array}$ & 3518 \\
\hline-------- & $\begin{array}{l}\text { A Conservative Selective Acknowledgment (SACK)-based } \\
\text { Loss Recovery Algorithm for TCP }\end{array}$ & 7 \\
\hline-------- & IMAP 4 Binary Content Extension & 3516 \\
\hline-------- & The Session Initiation Protocol (SIP) Refer Method & \\
\hline IPP $-E-T$ & Internet Printing Protocol/1.1: IPP URL Scheme & \\
\hline-------- & $\begin{array}{l}\text { Message Disposition Notification (MDN) profile for } \\
\text { Internet Message Access Protocol (IMAP) }\end{array}$ & 350 \\
\hline ---ー-ー-ー & $\begin{array}{l}\text { Internet Message Access Protocol (IMAP) - } \\
\text { MULTIAPPEND Extension }\end{array}$ & 3502 \\
\hline IMAPv 4 & INTERNET MESSAGE ACCESS PROTOCOL - VERSION 4 rev1 & \\
\hline
\end{tabular}




\begin{tabular}{|c|c|c|}
\hline------- & $\begin{array}{l}\text { Definitions of Managed Objects for Synchronous } \\
\text { Optical Network (SONET) Linear Automatic Protection } \\
\text { Switching (APS) Architectures }\end{array}$ & 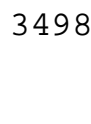 \\
\hline-- & $\begin{array}{l}\text { RTP Payload Format for Society of Motion Picture and } \\
\text { Television Engineers (SMPTE) } 292 \mathrm{M} \text { Video }\end{array}$ & 3497 \\
\hline-- & $\begin{array}{l}\text { Dynamic Host Configuration Protocol (DHCP) Option } \\
\text { for CableLabs Client Configuration }\end{array}$ & 3495 \\
\hline & $\begin{array}{l}\text { Punycode: A Bootstring encoding of Unicode for } \\
\text { Internationalized Domain Names in Applications (IDNA) }\end{array}$ & 2 \\
\hline-- & $\begin{array}{l}\text { Nameprep: A Stringprep Profile for Internationalized } \\
\text { Domain Names (IDN) }\end{array}$ & 491 \\
\hline------- & $\begin{array}{l}\text { Internationalizing Domain Names in Applications } \\
\text { (IDNA) }\end{array}$ & 490 \\
\hline & $\begin{array}{l}\text { STUN - Simple Traversal of User Datagram Protocol } \\
\text { (UDP) Through Network Address Translators (NATs) }\end{array}$ & 3489 \\
\hline ------- & Compressing the Session Initiation Protocol (SIP) & 86 \\
\hline-- & $\begin{array}{l}\text { The Session Initiation Protocol (SIP) and Session } \\
\text { Description Protocol (SDP) Static Dictionary for } \\
\text { Signaling Compression (SigComp) }\end{array}$ & \\
\hline & $\begin{array}{l}\text { Default Address Selection for Internet Protocol } \\
\text { version } 6 \text { (IPv6) }\end{array}$ & 484 \\
\hline-------- & $\begin{array}{l}\text { Signalling Unnumbered Links in CR-LDP } \\
\text { (Constraint-Routing Label Distribution Protocol) }\end{array}$ & 480 \\
\hline & $\begin{array}{l}\text { Fault Tolerance for the Label Distribution Protocol } \\
\text { (LDP) }\end{array}$ & 479 \\
\hline------ & $\begin{array}{l}\text { Graceful Restart Mechanism for Label Distribution } \\
\text { Protocol }\end{array}$ & 478 \\
\hline------ & $\begin{array}{l}\text { Signalling Unnumbered Links in Resource ReSerVation } \\
\text { Protocol - Traffic Engineering (RSVP-TE) }\end{array}$ & 3477 \\
\hline & $\begin{array}{l}\text { Generalized Multi-Protocol Label Switching (GMPLS) } \\
\text { Signaling Resource ReserVation Protocol-Traffic } \\
\text { Engineering (RSVP-TE) Extensions }\end{array}$ & 3473 \\
\hline------- & $\begin{array}{l}\text { Generalized Multi-Protocol Label Switching (GMPLS) } \\
\text { Signaling Constraint-based Routed Label Distribution } \\
\text { Protocol (CR-LDP) Extensions }\end{array}$ & 3472 \\
\hline ----ー--- & $\begin{array}{l}\text { Generalized Multi-Protocol Label Switching (GMPLS) } \\
\text { Signaling Functional Description }\end{array}$ & 3471 \\
\hline CIM & Policy Core Information Model (PCIM) Extensions & 3460 \\
\hline-------- & $\begin{array}{l}\text { Critical Content Multi-purpose Internet Mail } \\
\text { Extensions (MIME) Parameter }\end{array}$ & 3459 \\
\hline & Message Context for Internet Mail & \\
\hline------ & $\begin{array}{l}\text { Dynamic Host Configuration Protocol (DHCPV4) } \\
\text { Configuration of IPsec Tunnel Mode }\end{array}$ & 3456 \\
\hline-------- & $\begin{array}{l}\text { Preparation of Internationalized Strings } \\
\text { ("stringprep") }\end{array}$ & 3454 \\
\hline-------- & $\begin{array}{l}\text { TCP Friendly Rate Control (TFRC) : Protocol } \\
\text { Specification }\end{array}$ & \\
\hline
\end{tabular}




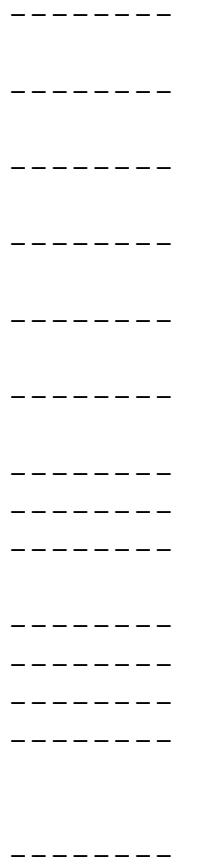

NAPTR

NAPTR

NAPTR
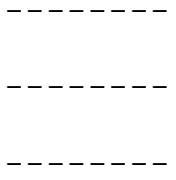

RMON-MIB
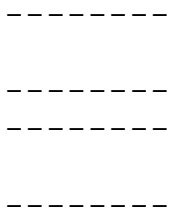

IPP $-E-T$

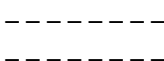

Time To Live (TTL) Processing in Multi-Protocol Label Switching (MPLS) Networks

The Classless Static Route Option for Dynamic Host Configuration Protocol (DHCP) version 4

Definitions of Extension Managed Objects for Asymmetric Digital Subscriber Lines

Layer-Two Tunneling Protocol Extensions for PPP Link Control Protocol Negotiation

Transport Layer Security over Stream Control Transmission Protocol

Remote Monitoring MIB Extensions for High Capacity Alarms

Entity Sensor Management Information Base

Network performance measurement with periodic streams

Session Initiation Protocol (SIP) Extension for Instant Messaging

obsoleting IQUERY

Internet Media Type message/sipfrag

Textual Conventions for Transport Addresses

Zero-byte Support for Bidirectional Reliable Mode $(\mathrm{R}$-mode) in Extended Link-Layer Assisted Robust Header Compression (ROHC) Profile

Session Description Protocol (SDP) Simple Capability Declaration

Dynamic Delegation Discovery System (DDDS) Part Four: The Uniform Resource Identifiers (URI)

Dynamic Delegation Discovery system (DDDS) Part Three: The Domain Name System (DNS) Database

Dynamic Delegation Discovery System (DDDS) Part Two: 3402 The Algorithm Integrated Services Digital Network (ISDN) User Part
(ISUP) to Session Initiation Protocol (SIP) Mapping

Dynamic Host Configuration Protocol (DHCP) Domain Search Option

Encoding Long Options in the Dynamic Host Configuration Protocol (DHCPV4)

Remote Network Monitoring MIB Protocol Identifier Reference Extensions

IP Packet Delay Variation Metric for IP Performance Metrics (IPPM)

Increasing $\mathrm{TCP}^{\prime} \mathrm{S}$ Initial Window

Real-time Transport Protocol (RTP) Payload for Comfort Noise (CN)

Grouping of Media Lines in the Session Description Protocol (SDP)

Internet Printing Protocol (IPP): The 'collection' attribute syntax

3443

3442

3434

3433

3432

3428

3425

3420

3419

3408

3407

3404

3403

3398

3397

3396

3395

3390

3389

3388

3382

RFC Editor

Informational

[Page 42] 


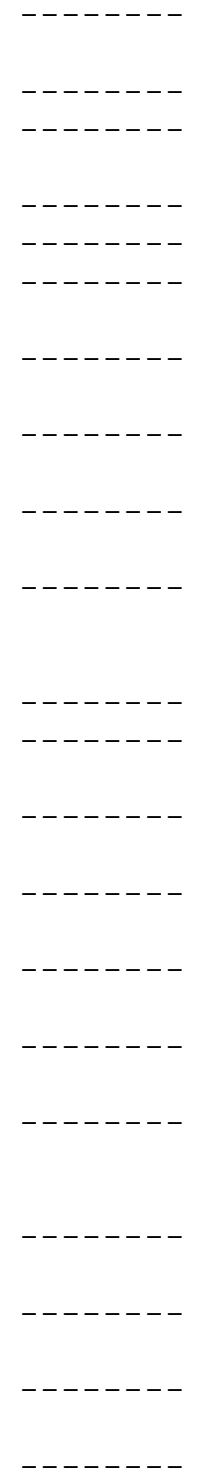

SIP

SIP

SIP

SIP
Integration of Resource Management and Session Initiation Protocol (SIP)

The Session Initiation Protocol (SIP) UPDATE Method

Layer Two Tunneling Protocol (L2TP) Differentiated Services Extension

Allocation Guidelines for IPv6 Multicast Addresses $\quad 3307$

Unicast-Prefix-based IPv6 Multicast Addresses 3306

Layer Two Tunnelling Protocol (L2TP): ATM access 3301 network extensions

Content Negotiation for Messaging Services based on Email

Named Subordinate References in Lightweight Directory Access Protocol (LDAP) Directories

Definitions of Managed Objects for the General Switch Management Protocol (GSMP)

General Switch Management Protocol (GSMP) Packet Encapsulations for Asynchronous Transfer Mode (ATM), Ethernet and Transmission Control Protocol (TCP)

General Switch Management Protocol (GSMP) V3

Management Information Base for the Differentiated Services Architecture

Remote Monitoring MIB Extensions for Differentiated Services

The VCDIFF Generic Differencing and Compression Data Format

An Internet Attribute Certificate Profile for Authorization

Internet X.509 Public Key Infrastructure Certificate and Certificate Revocation List (CRL) Profile

Algorithms and Identifiers for the Internet X.509 Public Key Infrastructure Certificate and Certificate Revocation List (CRL) Profile

Compressed Data Content Type for Cryptographic Message Syntax (CMS)

Remote Network Monitoring Management Information Base for High Capacity Networks

Multi-Protocol Label Switching (MPLS) Support of Differentiated Services

Advanced Encryption Standard (AES) Ciphersuites for Transport Layer Security (TLS)

Session Initiation Protocol (SIP)-Specific Event Notification

An Offer/Answer Model with Session Description Protocol (SDP)

Session Initiation Protocol (SIP): Locating SIP Servers

Reliability of Provisional Responses in Session Initiation Protocol (SIP)

3312

3311

3308

3306
3301

3297

3296

3295

3292

3289

3287

3284

3274

3273

3270

3268

3265

3264

3263

3262

RFC Editor 


\section{SIP}
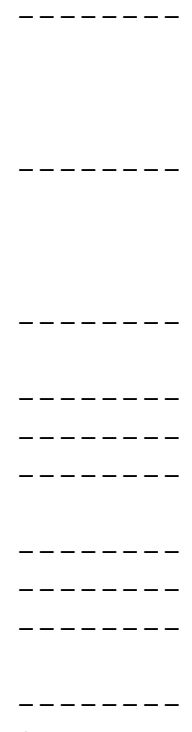

SLP
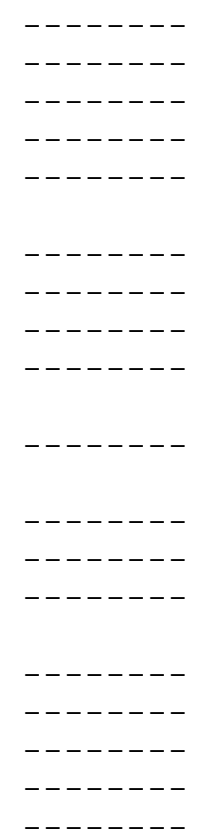
IPCOMP $--------$
SIP: Session Initiation Protocol

The DocSIS (Data-Over-Cable Service Interface Specifications) Device Class DHCP (Dynamic Host Configuration Protocol) Relay Agent Information Sub-option

Extending Point-to-Point Protocol (PPP) over Synchronous Optical NETwork/Synchronous Digital Hierarchy (SONET/SDH) with virtual concatenation, high order and low order payloads

Versioning Extensions to WebDAV (Web Distributed Authoring and Versioning)

An Expedited Forwarding PHB (Per-Hop Behavior)

Robust Header Compression (ROHC) over PPP

Definitions of Managed Objects for Scheduling Management Operations

Instance Digests in HTTP

Delta encoding in HTTP

DNSSEC and IPV6 A6 aware server/resolver message size requirements

Indicating Resolver Support of DNSSEC

Vendor Extensions for Service Location Protocol, Version 2

Telephony Routing over IP (TRIP) 3219

LSP Modification Using CR-LDP 3214

Constraint-Based LSP Setup using LDP 3212

RSVP-TE: Extensions to RSVP for LSP Tunnels 3209

SMTP Service Extension for Secure SMTP over 3207

Transport Layer Security

The SYS and AUTH POP Response Codes 3206

MIME media types for ISUP and QSIG Objects 3204

DHCP reconfigure extension 3203

Definitions of Managed Objects for Frame Relay 3202 Service Level Definitions

Definitions of Managed Objects for Circuit to 3201 Interface Translation

Reliable Delivery for syslog 3195

Securing L2TP using IPsec 3193

RTP Payload Format for 12-bit DAT Audio and 20- and 3190

24-bit Linear Sampled Audio

RTP Payload Format for DV (IEC 61834) Video 3189

Reuse of CMS Content Encryption Keys 3185

Identity Representation for RSVP 3182

Signaled Preemption Priority Policy Element 3181

Aggregation of RSVP for IPV4 and IPV6 Reservations 3175

IP Payload Compression Protocol (IPComp) 3173

The Addition of Explicit Congestion Notification 3168

$(\mathrm{ECN})$ to IP 


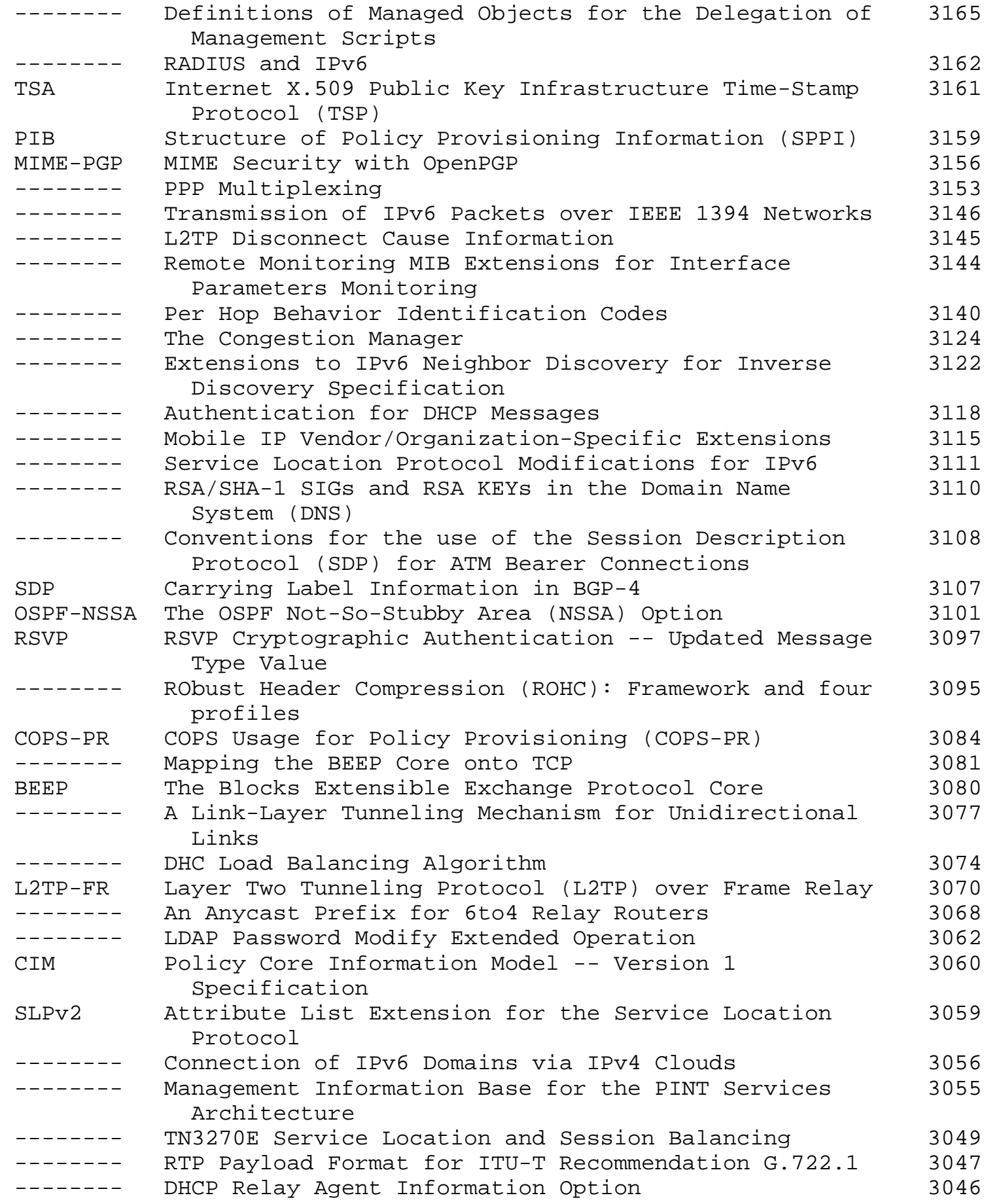




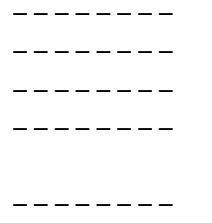

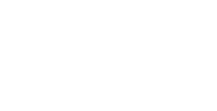

MPLS
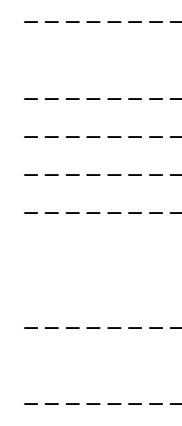

$------$

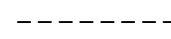

$-------$

$-------$
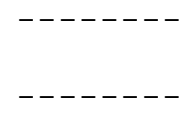

$-------$

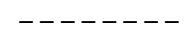

$------$
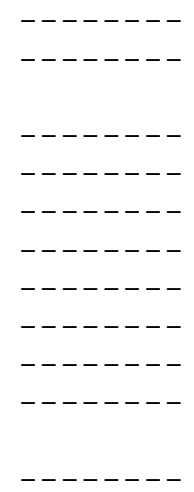

Enhancing TCP's Loss Recovery Using Limited Transmit VCID Notification over ATM link for LDP

MPLS using LDP and ATM VC Switching

Use of Label Switching on Frame Relay Networks Specification

The Assignment of the Information Field and Protocol Identifier in the Q.2941 Generic Identifier and Q.2957 User-to-user Signaling for the Internet Protocol

MPLS Label Stack Encoding

Multiprotocol Label Switching Architecture

SMTP Service Extensions for Transmission of Large and Binary MIME Messages

Reverse Tunneling for Mobile IP, revised 3024

XML Media Types

Using 31-Bit Prefixes on IPv4 Point-to-Point Links

Definitions of Managed Objects for Monitoring and Controlling the UNI/NNI Multilink Frame Relay Function

IP Version 6 Management Information Base for The Multicast Listener Discovery Protocol

XML DTD for Roaming Access Phone Book

RTP Payload Format for MPEG-4 Audio/Visual Streams Notification Log MIB

The IPv4 Subnet Selection Option for DHCP

Secure Domain Name System (DNS) Dynamic Update

Integrated Services in the Presence of Compressible Flows

The User Class Option for DHCP

The audio/mpeg Media Type

Specification of the Null Service Type

Format of the RSVP DCLASS object

Computing $\mathrm{TCP}^{\prime} \mathrm{S}$ Retransmission Timer

Registration of Charset and Languages Media Features Tags

Use of the CAST-128 Encryption Algorithm in CMS

Distributed Management Expression MIB

Event MIB

The SIP INFO Method

IMAP 4 ID extension

HTTP State Management Mechanism

RSVP Refresh Overhead Reduction Extensions

Real-Time Transport Protocol Management Information Base

Definitions of Managed objects for Monitoring and Controlling the Frame Relay/ATM PVC Service Interworking Function
3042

3038

3035

3034

3033

3032

3031

3030

3023

3021

3020

3019

3017

3016

3014

3011

3007

3006

3004

3003

2997

2996

2988

2987

2984

2982

2981

2976

2971

2965

2961

2959

2955 


\begin{tabular}{|c|c|c|}
\hline & $\begin{array}{l}\text { Generic Security Service API Version } 2 \text { : Java } \\
\text { Bindings }\end{array}$ & 28 \\
\hline----- & Deliver By SMTP Service Extension & 2852 \\
\hline LDIF & $\begin{array}{l}\text { The LDAP Data Interchange Format (LDIF) - Technical } \\
\text { Specification }\end{array}$ & 2849 \\
\hline-------- & $\begin{array}{l}\text { The PINT Service Protocol: Extensions to SIP and SDP } \\
\text { for IP Access to Telephone call Services }\end{array}$ & 2848 \\
\hline LIPKEY & $\begin{array}{l}\text { LIPKEY - A Low Infrastructure Public Key Mechanism } \\
\text { Using SPKM }\end{array}$ & 2847 \\
\hline-------- & GSTN Address Element Extensions in E-mail Services & 2846 \\
\hline TSIG & Secret Key Transaction Authentication for DNS (TSIG) & \\
\hline GSN & IP and ARP over HIPPI-6400 (GSN) & \\
\hline-------- & ARP and IP Broadcast over HIPPI-800 & 28 \\
\hline-------- & Using Digest Authentication as a SASL Mechanism & \\
\hline MAIL & Internet Message Format & 2822 \\
\hline-------- & Upgrading to TLS Within HTTP/1.1 & \\
\hline-------- & Integrated Service Mappings on IEEE 802 Networks & \\
\hline-------- & $\begin{array}{l}\text { SBM (Subnet Bandwidth Manager): A Protocol for } \\
\text { RSVP-based Admission Control over IEEE 802-style } \\
\text { networks }\end{array}$ & \\
\hline-------- & Certificate Management Messages over CMS & 2797 \\
\hline-------- & $\begin{array}{l}\text { Mobile IP Network Access Identifier Extension for } \\
\text { IPv4 }\end{array}$ & 279 \\
\hline-------- & Mail Monitoring MIB & 2789 \\
\hline-------- & Network Services Monitoring MIB & 27 \\
\hline-------- & $\begin{array}{l}\text { Definitions of Managed Objects for the Virtual } \\
\text { Router Redundancy Protocol }\end{array}$ & 2787 \\
\hline GRE & Generic Routing Encapsulation (GRE) & 2784 \\
\hline DNS-SRV & $\begin{array}{l}\text { A DNS RR for specifying the location of services } \\
\text { (DNS SRV) }\end{array}$ & 2782 \\
\hline MZAP & Multicast-Scope Zone Announcement Protocol (MZAP) & 2776 \\
\hline RPSL & Routing Policy System Replication & 2769 \\
\hline SIIT & Stateless IP/ICMP Translation Algorithm (SIIT) & 2765 \\
\hline-------- & RSVP Extensions for Policy Control & 2750 \\
\hline-------- & COPS usage for RSVP & 2749 \\
\hline COPS & The COPS (Common Open Policy Service) Protocol & 2748 \\
\hline-------- & RSVP Cryptographic Authentication & 2747 \\
\hline-------- & RSVP Operation Over IP Tunnels & 2746 \\
\hline-------- & RSVP Diagnostic Messages & 2745 \\
\hline-------- & Generic Security Service API Version 2 : C-bindings & 2744 \\
\hline-------- & $\begin{array}{l}\text { Generic Security Service Application Program } \\
\text { Interface Version 2, Update } 1\end{array}$ & 2743 \\
\hline --------- & OSPF for IPV6 & 2740 \\
\hline-------- & Calendar Attributes for vCard and LDAP & 2739 \\
\hline-------- & $\begin{array}{l}\text { Corrections to "A Syntax for Describing Media } \\
\text { Feature sets" }\end{array}$ & 2738 \\
\hline -------- & NHRP Support for Virtual Private Networks & 2735 \\
\hline-------- & IPv4 over IEEE 1394 & 2734 \\
\hline
\end{tabular}




\begin{tabular}{|c|c|}
\hline MADCAP & $\begin{array}{l}\text { Multicast Address Dynamic Client Allocation Protocol } \\
\text { (MADCAP) }\end{array}$ \\
\hline-------- & $\begin{array}{l}\text { The Transmission of IP Over the Vertical Blanking } \\
\text { Interval of a Television Signal }\end{array}$ \\
\hline-------- & PGP Authentication for RIPE Database Updates \\
\hline-------- & Routing Policy System Security \\
\hline-------- & Traffic Flow Measurement: Meter MIB \\
\hline TLS & $\begin{array}{l}\text { Addition of Kerberos Cipher Suites to Transport } \\
\text { Layer Security (TLS) }\end{array}$ \\
\hline-------- & IPv6 Router Alert Option \\
\hline MLD-IPv 6 & Multicast Listener Discovery (MLD) for IPv6 \\
\hline-------- & Integrated Services Mappings for Low Speed Networks \\
\hline-------- & PPP in a Real-time Oriented HDLC-like Framing \\
\hline-------- & The Multi-Class Extension to Multi-Link PPP \\
\hline VPNI & Virtual Private Networks Identifier \\
\hline-------- & $\begin{array}{l}\text { Multiprotocol Encapsulation over ATM Adaptation } \\
\text { Layer } 5\end{array}$ \\
\hline-------- & A Round-trip Delay Metric for IPPM \\
\hline-------- & A One-way Packet Loss Metric for IPPM \\
\hline-------- & A One-way Delay Metric for IPPM \\
\hline IPPM-MET & IPPM Metrics for Measuring Connectivity \\
\hline NHRP-MIB & $\begin{array}{l}\text { Definitions of Managed Objects for the NBMA Next Hop } \\
\text { Resolution Protocol (NHRP) }\end{array}$ \\
\hline-------- & IPv6 Jumbograms \\
\hline-------- & Non-Terminal DNS Name Redirection \\
\hline EDNSO & Extension Mechanisms for DNS (EDNSO) \\
\hline-------- & Definitions of Managed Objects for the ADSL Lines \\
\hline L2TP & Layer Two Tunneling Protocol "L2TP" \\
\hline-------- & RTP Payload Format for PureVoice(tm) Audio \\
\hline-------- & CIP Transport Protocols \\
\hline-------- & $\begin{array}{l}\text { MIME Object Definitions for the Common Indexing } \\
\text { Protocol (CIP) }\end{array}$ \\
\hline CIP & $\begin{array}{l}\text { The Architecture of the Common Indexing Protocol } \\
\text { (CIP) }\end{array}$ \\
\hline ODMR-SMTP & $\begin{array}{l}\text { ON-DEMAND MAIL RELAY (ODMR) SMTP with Dynamic IP } \\
\text { Addresses }\end{array}$ \\
\hline-------- & Internationalization of the File Transfer Protocol \\
\hline-------- & Enhanced Security Services for S/MIME \\
\hline-------- & Diffie-Hellman Key Agreement Method \\
\hline-------- & $\begin{array}{l}\text { NFS Version } 2 \text { and Version } 3 \text { Security Issues and the } \\
\text { NFS Protocol's Use of RPCSEC_GSS and Kerberos V5 }\end{array}$ \\
\hline RPSL & Routing Policy Specification Language (RPSL) \\
\hline-------- & PPP over SONET/SDH \\
\hline-------- & DHCP Options for Service Location Protocol \\
\hline-------- & Service Templates and Service: Schemes \\
\hline-------- & Service Location Protocol, Version 2 \\
\hline-------- & Directory Server Monitoring MIB \\
\hline-------- & ILMI-Based Server Discovery for NHRP \\
\hline
\end{tabular}




\begin{tabular}{|c|c|c|}
\hline--- & ILMI-Based Server Discovery for MARS & 2602 \\
\hline--- & ILMI-Based Server Discovery for ATMARP & 2601 \\
\hline ------- & Assured Forwarding PHB Group & 2597 \\
\hline------- & Using TLS with IMAP, POP 3 and ACAP & 5 \\
\hline------- & Definitions of Managed Objects for WWW Services & \\
\hline------- & $\begin{array}{l}\text { Transmission of IPv6 Packets over Frame Relay } \\
\text { Networks specification }\end{array}$ & \\
\hline LDAPv3 & $\begin{array}{l}\text { Lightweight Directory Access Protocol (v3): } \\
\text { Extensions for Dynamic Directory Services }\end{array}$ & \\
\hline------- & $\begin{array}{l}\text { Internet X.509 Public Key Infrastructure Operational } \\
\text { Protocols: FTP and HTTP }\end{array}$ & \\
\hline------- & $\begin{array}{l}\text { Definitions of Managed Objects for APPN/HPR in IP } \\
\text { Networks }\end{array}$ & 2584 \\
\hline $\mathrm{TCP}-\mathrm{CC}$ & TCP Congestion Control & \\
\hline PP-MIB & Application Management MIB & \\
\hline------- & $\begin{array}{l}\text { DHCP Option to Disable Stateless Auto-Configuration } \\
\text { in IPv4 Clients }\end{array}$ & \\
\hline TN2370E-RT & $\begin{array}{l}\text { Definitions of Protocol and Managed Objects for } \\
\text { TN3270E Response Time Collection Using SMIv2 } \\
\text { (TN3270E-RT-MIB) }\end{array}$ & \\
\hline------- & $\begin{array}{l}\text { Base Definitions of Managed Objects for TN3270E } \\
\text { Using SMIv2 }\end{array}$ & \\
\hline PKIX & $\begin{array}{l}\text { X.509 Internet Public Key Infrastructure Online } \\
\text { Certificate Status Protocol - OCSP }\end{array}$ & \\
\hline HTML & $\begin{array}{l}\text { MIME Encapsulation of Aggregate Documents, such as } \\
\text { HTML (MHTML) }\end{array}$ & \\
\hline------- & $\begin{array}{l}\text { Use of BGP-4 Multiprotocol Extensions for IPv6 } \\
\text { Inter-Domain Routing }\end{array}$ & \\
\hline DHK-DNS & $\begin{array}{l}\text { Storage of Diffie-Hellman Keys in the Domain Name } \\
\text { System (DNS) }\end{array}$ & \\
\hline------- & DSA KEYs and SIGs in the Domain Name System (DNS) & 6 \\
\hline------- & Media Features for Display, Print, and Fax & \\
\hline------- & A Syntax for Describing Media Feature Sets & \\
\hline------- & Extended Facsimile Using Internet Mail & \\
\hline------- & $\begin{array}{l}\text { Indicating Supported Media Features Using Extensions } \\
\text { to DSN and MDN }\end{array}$ & \\
\hline------- & $\begin{array}{l}\text { Transmission of IPv6 over IPv4 Domains without } \\
\text { Explicit Tunnels }\end{array}$ & \\
\hline-------- & Reserved IPv6 Subnet Anycast Addresses & \\
\hline ATM-MIBMAN & Definitions of Managed Objects for ATM Management & \\
\hline ATM-TC-OID & $\begin{array}{l}\text { Definitions of Textual Conventions and } \\
\text { OBJECT-IDENTITIES for ATM Management }\end{array}$ & \\
\hline------- & $\begin{array}{l}\text { Managed Objects for Controlling the Collection and } \\
\text { Storage of Accounting Information for } \\
\text { Connection-Oriented Networks }\end{array}$ & \\
\hline------- & Accounting Information for ATM Networks & \\
\hline------- & Compressing IP/UDP/RTP Headers for Low-Speed Serial & \\
\hline
\end{tabular}
Links 


\begin{tabular}{|c|c|c|}
\hline-- & IP Header Compression & \\
\hline & Transmission of IPv6 Packets over ARCnet Networks & 497 \\
\hline-- & $\begin{array}{l}\text { Definitions of Managed Objects for the DSO and DSO } \\
\text { Bundle Interface Type }\end{array}$ & \\
\hline IPv6ATMNET & IPv6 over ATM Networks & 2 \\
\hline IPv6-NBMA & $\begin{array}{l}\text { IPv6 over Non-Broadcast Multiple Access (NBMA) } \\
\text { networks }\end{array}$ & \\
\hline-------- & $\begin{array}{l}\text { DHCP Option for The Open Group's User Authentication } \\
\text { Protocol }\end{array}$ & \\
\hline-------- & PPP LCP Internationalization Configuration Option & \\
\hline-------- & Gateways and MIME Security Multiparts & \\
\hline-------- & $\begin{array}{l}\text { Definition of the Differentiated Services Field (DS } \\
\text { Field) in the IPv4 and IPv6 Headers }\end{array}$ & \\
\hline-------- & Generic Packet Tunneling in IPv6 Specification & \\
\hline-------- & Transmission of IPv6 Packets over Token Ring Networks & \\
\hline-------- & Transmission of IPv6 Packets over FDDI Networks & \\
\hline-------- & Transmission of IPv6 Packets over Ethernet Networks & \\
\hline EBN-MIB & $\begin{array}{l}\text { Definitions of Managed Objects for Extended Border } \\
\text { Node }\end{array}$ & \\
\hline-------- & Definitions of Managed Objects for APPN TRAPS & \\
\hline APPN-MIB & Definitions of Managed Objects for APPN & \\
\hline-------- & The ESP CBC-Mode Cipher Algorithms & \\
\hline POP 3-EXT & POP3 Extension Mechanism & \\
\hline IMIP & $\begin{array}{l}\text { iCalendar Message-Based Interoperability Protocol } \\
\text { (iMIP) }\end{array}$ & \\
\hline ITIP & $\begin{array}{l}\text { iCalendar Transport-Independent Interoperability } \\
\text { Protocol (iTIP) Scheduling Events, BusyTime, To-dos } \\
\text { and Journal Entries }\end{array}$ & \\
\hline ICALENDAR & $\begin{array}{l}\text { Internet Calendaring and Scheduling Core object } \\
\text { Specification (iCalendar) }\end{array}$ & \\
\hline OTP-SASL & The One-Time-Password SASL Mechanism & \\
\hline-------- & BGP Route Flap Damping & \\
\hline-------- & RTP Payload Format for JPEG-compressed Video & \\
\hline-------- & RTP Payload Format for BT.656 Video Encoding & \\
\hline-------- & FTP Extensions for IPv6 and NATs & \\
\hline MIME-VCARD & vCard MIME Directory Profile & \\
\hline TXT-DIR & A MIME Content-Type for Directory Information & \\
\hline 3DESE & The PPP Triple-DES Encryption Protocol (3DESE) & \\
\hline DESE-bis & The PPP DES Encryption Protocol, Version 2 (DESE-bis) & \\
\hline-------- & $\begin{array}{l}\text { Definitions of Managed Objects for Multicast over } \\
\text { UNI 3.0/3.1 based ATM Networks }\end{array}$ & \\
\hline-------- & The NULL Encryption Algorithm and Its Use With IPsec & \\
\hline ESPDES-CBC & The ESP DES-CBC Cipher Algorithm With Explicit IV & \\
\hline-------- & The Use of HMAC-SHA-1-96 within ESP and AH & \\
\hline-------- & The Use of HMAC-MD5-96 within ESP and AH & \\
\hline DATA-URL & URL scheme & \\
\hline CIDMID-URL & Content-ID and Message-ID Uniform Resource Locators & \\
\hline
\end{tabular}




\begin{tabular}{|c|c|c|}
\hline---- & $\begin{array}{l}\text { eature negotiation mechanism for the File Transfer } \\
\text { Protocol }\end{array}$ & \\
\hline-------- & Returning Values from Forms: multipart/form-data & 388 \\
\hline MIME-RELAT & The MIME Multipart/Related Content-type & 387 \\
\hline-------- & $\begin{array}{l}\text { Protection of BGP Sessions via the TCP MD } 5 \text { Signature } \\
\text { Option }\end{array}$ & 385 \\
\hline POP-URL & POP URL Scheme & 2384 \\
\hline-------- & $\begin{array}{l}\text { Interoperation of Controlled-Load Service and } \\
\text { Guaranteed Service with ATM }\end{array}$ & \\
\hline-------- & RSVP over ATM Implementation Requirements & \\
\hline TIPV3 & Transaction Internet Protocol Version 3.0 & \\
\hline OSPF-LSA & The OSPF Opaque LSA Option & \\
\hline-------- & $\begin{array}{l}\text { The Use of URLs as Meta-Syntax for Core Mail List } \\
\text { Commands and their Transport through Message Header } \\
\text { Fields }\end{array}$ & 9 \\
\hline URLMAILTO & The mailto URL scheme & \\
\hline PPP & PPP Over AAL5 & \\
\hline PPP-FUNI & PPP Over FUNI & \\
\hline IMAP 4 & IMAP 4 Namespace & \\
\hline NHRP-SCSP & A Distributed NHRP Service Using SCSP & \\
\hline SCSP & Server Cache Synchronization Protocol (SCSP) & \\
\hline-------- & NHRP Protocol Applicability Statement & \\
\hline NHRP & NBMA Next Hop Resolution Protocol (NHRP) & \\
\hline UNI-SIG & $\begin{array}{l}\text { ATM Signalling Support for IP over ATM - UNI } \\
\text { Signalling } 4.0 \text { Update }\end{array}$ & \\
\hline RTSP & Real Time Streaming Protocol (RTSP) & \\
\hline IPOA-MIB & $\begin{array}{l}\text { Definitions of Managed Objects for Classical IP and } \\
\text { ARP Over ATM Using SMIv2 (IPOA-MIB) }\end{array}$ & \\
\hline DNS-NCACHE & Negative Caching of DNS Queries (DNS NCACHE) & \\
\hline $\mathrm{OR}-\mathrm{ADD}$ & $\begin{array}{l}\text { Representing the O/R Address hierarchy in the } \mathrm{X} .500 \\
\text { Directory Information Tree }\end{array}$ & 229 \\
\hline SUBTABLE & $\begin{array}{l}\text { Representing Tables and Subtrees in the X.500 } \\
\text { Directory }\end{array}$ & 229 \\
\hline-------- & Mobile-IPv4 Configuration Option for PPP IPCP & \\
\hline SLM-APP & $\begin{array}{l}\text { Definitions of System-Level Managed Objects for } \\
\text { Applications }\end{array}$ & 2287 \\
\hline-------- & $\begin{array}{l}\text { Definitions of Managed Objects for IEEE } 802.12 \\
\text { Repeater Devices }\end{array}$ & 2266 \\
\hline RTP-MPEG & RTP Payload Format for MPEG1/MPEG2 Video & \\
\hline-------- & Using Domains in LDAP/X.500 Distinguished Names & \\
\hline ACAP & ACAP -- Application Configuration Access Protocol & 2244 \\
\hline OTP-ER & OTP Extended Responses & \\
\hline NETWAREIP & NetWare/IP Domain Name and Information & \\
\hline DHCP-NDS & DHCP Options for Novell Directory Services & 2241 \\
\hline HPR-MIB & Definitions of Managed Objects for HPR using SMIv2 & \\
\hline DLUR-MIB & Definitions of Managed Objects for DLUR using SMIv2 & 223 \\
\hline MIME-EXT & MIME Parameter Value and Encoded Word Extensions: & 2231 \\
\hline
\end{tabular}




\begin{tabular}{|c|c|c|}
\hline FTP SECEXT & Security Extensions & 2228 \\
\hline-------- & Simple Hit-Metering and Usage-Limiting for HTTP & 2227 \\
\hline-------- & IP Broadcast over ATM Networks & \\
\hline IP-ATM & Classical IP and ARP over ATM & \\
\hline IMAP 4 LOGIN & IMAP 4 Login Referrals & \\
\hline-------- & A Common Schema for the Internet White Pages Service & \\
\hline-------- & $\begin{array}{l}\text { General Characterization Parameters for Integrated } \\
\text { Service Network Elements }\end{array}$ & \\
\hline-------- & $\begin{array}{l}\text { Integrated Services Management Information Base } \\
\text { Guaranteed Service Extensions using SMIv2 }\end{array}$ & \\
\hline-------- & $\begin{array}{l}\text { Integrated Services Management Information Base } \\
\text { using SMIv2 }\end{array}$ & \\
\hline GQOS & Specification of Guaranteed Quality of Service & \\
\hline-------- & $\begin{array}{l}\text { Specification of the Controlled-Load Network Element } \\
\text { Service }\end{array}$ & \\
\hline RSVP-IS & The Use of RSVP with IETF Integrated Services & \\
\hline RSVP-IPSEC & RSVP Extensions for IPSEC Data Flows & \\
\hline RSVP-MIB & RSVP Management Information Base using SMIv2 & \\
\hline RSVP & $\begin{array}{l}\text { Resource ReSerVation Protocol (RSVP) }-- \text { Version } 1 \\
\text { Functional Specification }\end{array}$ & \\
\hline RPCSEC-GSS & RPCSEC_GSS Protocol Specification & \\
\hline $\mathrm{RTP}-\mathrm{RAD}$ & RTP Payload for Redundant Audio Data & \\
\hline IMAPPOPAU & $\begin{array}{l}\text { IMAP/POP AUTHorize Extension for Simple } \\
\text { Challenge/Response }\end{array}$ & \\
\hline IMAP 4MAIL & IMAP 4 Mailbox Referrals & \\
\hline-------- & $\begin{array}{l}\text { Communicating Presentation Information in Internet } \\
\text { Messages: The Content-Disposition Header Field }\end{array}$ & \\
\hline DNS-CLAR & Clarifications to the DNS Specification & \\
\hline IMAP 4-IDLE & IMAP 4 IDLE command & \\
\hline SLP & Service Location Protocol & \\
\hline-------- & $\begin{array}{l}\text { Use of an X.500/LDAP directory to support MIXER } \\
\text { address mapping }\end{array}$ & \\
\hline DNS-MCGAM & $\begin{array}{l}\text { Using the Internet DNS to Distribute MIXER } \\
\text { Conformant Global Address Mapping (MCGAM) }\end{array}$ & \\
\hline-------- & Carrying PostScript in X.400 and MIME & \\
\hline-------- & A MIME Body Part for FAX & \\
\hline-------- & X.400 Image Body Parts & \\
\hline-------- & Mapping between X.400 and RFC-822/MIME Message Bodies & \\
\hline MIXER & $\begin{array}{l}\text { MIXER (Mime Internet X.400 Enhanced Relay): Mapping } \\
\text { between X.400 and RFC } 822 / \mathrm{MIME}\end{array}$ & \\
\hline MAIL-SERV & $\begin{array}{l}\text { Mailbox Names for Common Services, Roles and } \\
\text { Functions }\end{array}$ & \\
\hline URN-SYNTAX & URN Syntax & \\
\hline DNS-UPDATE & $\begin{array}{l}\text { Dynamic Updates in the Domain Name System (DNS } \\
\text { UPDATE) }\end{array}$ & \\
\hline DC-MIB & Dial Control Management Information Base using SMIv2 & \\
\hline ISDN-MIB & ISDN Management Information Base using SMIv2 & \\
\hline ITOT & ISO Transport Service on top of TCP (ITOT) & \\
\hline
\end{tabular}




\begin{tabular}{|c|c|c|}
\hline $\mathrm{BAP}-\mathrm{BACP}$ & $\begin{array}{l}\text { The PPP Bandwidth Allocation Protocol (BAP) / The } \\
\text { PPP Bandwidth Allocation Control Protocol (BACP) }\end{array}$ & 2125 \\
\hline VEMMI-URL & VEMMI URL Specification & 122 \\
\hline ROUT-ALERT & IP Router Alert Option & 2113 \\
\hline $802.3-\mathrm{MIB}$ & $\begin{array}{l}\text { Definitions of Managed Objects for IEEE } 802.3 \\
\text { Repeater Devices using SMIv2 }\end{array}$ & 8 \\
\hline $\mathrm{PPP}-\mathrm{NBFCP}$ & The PPP NetBIOS Frames Control Protocol (NBFCP) & \\
\hline RIP-TRIG & $\begin{array}{l}\text { Triggered Extensions to RIP to Support Demand } \\
\text { Circuits }\end{array}$ & 1 \\
\hline IMAP 4-LIT & IMAP 4 non-synchronizing literals & \\
\hline IMAP 4-QUO & IMAP 4 QUOTA extension & 208 \\
\hline HMAC-MD 5 & HMAC-MD5 IP Authentication with Replay Prevention & \\
\hline RIPNG-IPV6 & RIPng for IPv6 & 208 \\
\hline URI-ATT & $\begin{array}{l}\text { Definition of an X.500 Attribute Type and an Object } \\
\text { Class to Hold Uniform Resource Identifiers (URIs) }\end{array}$ & \\
\hline MIME-MODEL & $\begin{array}{l}\text { The Model Primary Content Type for Multipurpose } \\
\text { Internet Mail Extensions }\end{array}$ & 2077 \\
\hline URLZ39.50 & Uniform Resource Locators for Z39.50 & 2056 \\
\hline SNANAU-APP & Definitions of Managed Objects for APPC using SMIv2 & \\
\hline $\mathrm{PPP}-\mathrm{SNACP}$ & The PPP SNA Control Protocol (SNACP) & $20<$ \\
\hline SMTP-ENH & $\begin{array}{l}\text { SMTP Service Extension for Returning Enhanced Error } \\
\text { Codes }\end{array}$ & \\
\hline RTP-CELLB & RTP Payload Format of Sun's CellB Video Encoding & 2029 \\
\hline SPKM & The Simple Public-Key GSS-API Mechanism (SPKM) & \\
\hline DLSW-MIB & $\begin{array}{l}\text { Definitions of Managed Objects for Data Link } \\
\text { Switching using SMIv2 }\end{array}$ & 202 \\
\hline MULTI-UNI & $\begin{array}{l}\text { Support for Multicast over UNI } 3.0 / 3.1 \text { based ATM } \\
\text { Networks }\end{array}$ & 202 \\
\hline $802.12-\mathrm{MIB}$ & IEEE 802.12 Interface MIB & 2020 \\
\hline $\mathrm{TCP}-\mathrm{ACK}$ & TCP Selective Acknowledgment Options & 201 \\
\hline $\mathrm{URL}-\mathrm{ACC}$ & Definition of the URL MIME External-Body Access-Type & \\
\hline MIME-PGP & MIME Security with Pretty Good Privacy (PGP) & 20 \\
\hline MOBILEIPMI & $\begin{array}{l}\text { The Definitions of Managed objects for IP Mobility } \\
\text { Support using SMIv2 }\end{array}$ & 200 \\
\hline-------- & Applicability Statement for IP Mobility Support & 200 \\
\hline MINI-IP & Minimal Encapsulation within IP & 2004 \\
\hline IPENCAP IP & IP Encapsulation within IP & 200 \\
\hline $\mathrm{BGP}-\mathrm{COMM}$ & BGP Communities Attribute & 1997 \\
\hline DNS-NOTIFY & $\begin{array}{l}\text { A Mechanism for Prompt Notification of Zone Changes } \\
\text { (DNS NOTIFY) }\end{array}$ & 1996 \\
\hline DNS-IZT & Incremental Zone Transfer in DNS & 1995 \\
\hline SMTP-ETRN & $\begin{array}{l}\text { SMTP Service Extension for Remote Message Queue } \\
\text { Starting }\end{array}$ & 1985 \\
\hline SNA & Serial Number Arithmetic & 1982 \\
\hline PPP-FRAME & $P P P$ in Frame Relay & 1973 \\
\hline $\mathrm{PPP}-\mathrm{ECP}$ & The PPP Encryption Control Protocol (ECP) & 1968 \\
\hline GSSAP I-KER & The Kerberos Version 5 GSS-API Mechanism & 1964 \\
\hline $\mathrm{PPP}-\mathrm{CCP}$ & The PPP Compression Control Protocol (CCP) & 1962 \\
\hline
\end{tabular}




\begin{tabular}{|c|c|}
\hline AUTH-SOCKS & Username/Password Authentication for SOCKS V5 \\
\hline SOCKSV5 & SOCKS Protocol Version 5 \\
\hline MIME-Encyp & $\begin{array}{l}\text { Security Multiparts for MIME: Multipart/Signed and } \\
\text { Multipart/Encrypted }\end{array}$ \\
\hline------- & Binding Protocols for ONC RPC Version 2 \\
\hline$P C$ & $\begin{array}{l}\text { RPC: Remote Procedure Call Protocol Specification } \\
\text { Version } 2\end{array}$ \\
\hline----- & The ESP DES-CBC Transform \\
\hline------- & Requirements for IP Version 4 Routers \\
\hline$S P F-D C$ & Extending OSPF to Support Demand Circuits \\
\hline IME-EDI & MIME Encapsulation of EDI Objects \\
\hline TM & ATM Signaling Support for IP over ATM \\
\hline PNG & $\begin{array}{l}\text { The Recommendation for the IP Next Generation } \\
\text { Protocol }\end{array}$ \\
\hline acMIME & MIME Encapsulation of Macintosh Files - MacMIME \\
\hline MAP 4-AUTH & IMAP 4 Authentication Mechanisms \\
\hline DBMS-MIB & $\begin{array}{l}\text { Relational Database Management System (RDBMS) } \\
\text { Management Information Base (MIB) using SMIv2 }\end{array}$ \\
\hline PP-TRANS & PPP Reliable Transmission \\
\hline PP-ISDN & PPP over ISDN \\
\hline$P-X 25$ & $\mathrm{PPP}$ in $\mathrm{X} .25$ \\
\hline $\mathrm{IP}-\mathrm{DC}$ & Extensions to RIP to Support Demand Circuits \\
\hline OPT-ENVIR & Telnet Environment Option \\
\hline$P P-L C P$ & PPP LCP Extensions \\
\hline PP / IPMIB & $\begin{array}{l}\text { The Definitions of Managed objects for the IP } \\
\text { Network Control Protocol of the Point-to-Point } \\
\text { Protocol }\end{array}$ \\
\hline $\mathrm{PP} / \mathrm{SECMIB}$ & $\begin{array}{l}\text { The Definitions of Managed objects for the Security } \\
\text { Protocols of the Point-to-Point Protocol }\end{array}$ \\
\hline $\mathrm{PP} / \mathrm{LCPMIB}$ & $\begin{array}{l}\text { The Definitions of Managed Objects for the Link } \\
\text { Control Protocol of the Point-to-Point Protocol }\end{array}$ \\
\hline NMP - IPX & SNMP over IPX \\
\hline ENT & Identification Protocol \\
\hline $\mathrm{NMP}-\mathrm{X} .25$ & SNMP MIB Extension for the X.25 Packet Layer \\
\hline JMP - LAPB & SNMP MIB Extension for X.25 LAPB \\
\hline $\mathrm{P}-\mathrm{OSINLC}$ & The PPP OSI Network Layer Control Protocol (OSINLCP) \\
\hline $\mathrm{PT}-\mathrm{RFC}$ & Telnet Remote Flow Control Option \\
\hline$P-I P C P$ & The PPP Internet Protocol Control Protocol (IPCP) \\
\hline $\mathrm{CP}-\mathrm{EXT}$ & TCP Extensions for High Performance \\
\hline------- & $\begin{array}{l}\text { Encoding Network Addresses to Support Operation over } \\
\text { Non-OSI Lower Layers }\end{array}$ \\
\hline $\mathrm{CMP}-\mathrm{ROUT}$ & ICMP Router Discovery Messages \\
\hline -IS & $\begin{array}{l}\text { Use of OSI IS-IS for routing in TCP/IP and dual } \\
\text { environments }\end{array}$ \\
\hline$-\mathrm{CMPRS}$ & Compressing TCP/IP Headers for Low-Speed Serial Links \\
\hline $\mathrm{PT}-\mathrm{XDL}$ & Telnet X display location option \\
\hline PT-TERM & Telnet terminal-type option \\
\hline
\end{tabular}


3.5. Best Current Practice Ordered by $\mathrm{BCP}$

[Note: an asterisk at the end of a line indicates a change from the previous edition of this document.]

\begin{tabular}{|c|c|c|c|}
\hline Mnemonic & Title & $\mathrm{RFC \#}$ & CP \# \\
\hline-------- & $\begin{array}{l}\text { Variance for The PPP Compression Control Protocol } \\
\text { and The PPP Encryption Control Protocol }\end{array}$ & 1915 & 3 \\
\hline-------- & $\begin{array}{c}\text { An Appeal to the Internet Community to Return } \\
\text { Unused IP Networks (Prefixes) to the IANA }\end{array}$ & 1917 & 4 \\
\hline-------- & Address Allocation for Private Internets & 1918 & 5 \\
\hline---- & $\begin{array}{l}\text { Guidelines for creation, selection, and } \\
\text { registration of an Autonomous System (AS) }\end{array}$ & 1930 & \\
\hline -------- & $\begin{array}{l}\text { Implications of Various Address Allocation } \\
\text { Policies for Internet Routing }\end{array}$ & 2008 & 7 \\
\hline-------- & IRTF Research Group Guidelines and Procedures & 2014 & 8 \\
\hline-------- & The Internet Standards Process -- Revision 3 & 2026 & 9 \\
\hline-------- & $\begin{array}{l}\text { IAB and IESG Selection, Confirmation, and Recall } \\
\text { Process: Operation of the Nominating and Recall } \\
\text { Committees }\end{array}$ & 3777 & 10 \\
\hline-------- & $\begin{array}{l}\text { The Organizations Involved in the IETF Standards } \\
\text { Process }\end{array}$ & 2028 & 11 \\
\hline-------- & Internet Registry IP Allocation Guidelines & 2050 & 12 \\
\hline-------- & $\begin{array}{l}\text { Media Type Specifications and Registration } \\
\text { Procedures }\end{array}$ & $4288 *$ & 13 \\
\hline-------- & $\begin{array}{l}\text { Multipurpose Internet Mail Extensions (MIME) Part } \\
\text { Four: Registration Procedures }\end{array}$ & $4289 *$ & 13 \\
\hline
\end{tabular}




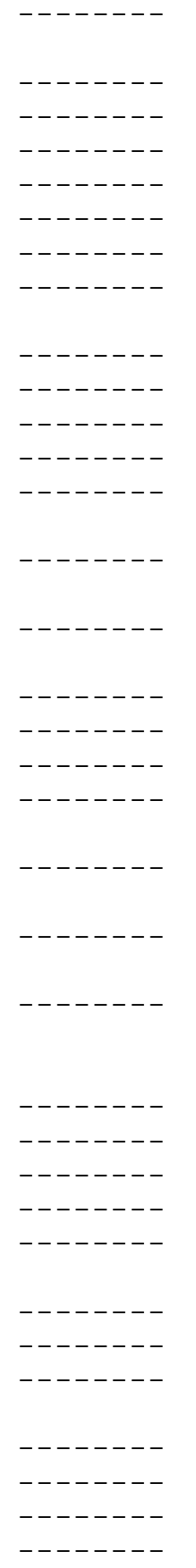

Key words for use in RFCs to Indicate Requirement Levels

Deployment of the Internet White Pages Service Selection and Operation of Secondary DNS Servers Use of DNS Aliases for Network Services
$2119 \quad 14$

$2148 \quad 15$

$2182 \quad 16$

$2219 \quad 17$

$2277 \quad 18$

$2978 \quad 19$

$2317 \quad 20$

$2350 \quad 21$

$2360 \quad 22$

$2365 \quad 23$

$2379 \quad 24$

$2418 \quad 25$

$2434 \quad 26$

$2438 \quad 27$

$2488 \quad 28$

$2505 \quad 30$

$2506 \quad 31$

$2606 \quad 32$

$2644 \quad 34$

$2736 \quad 36$

$2780 \quad 37$

$2827 \quad 38$

$2850 \quad 39$

$2870 \quad 40$

$2914 \quad 41$

$2929 \quad 42$

293943

$2964 \quad 44$

$3005 \quad 45$

$3013 \quad 46$

4646 * 47

$4647 * \quad 47$

$3150 \quad 48$

$3155 \quad 50$ 


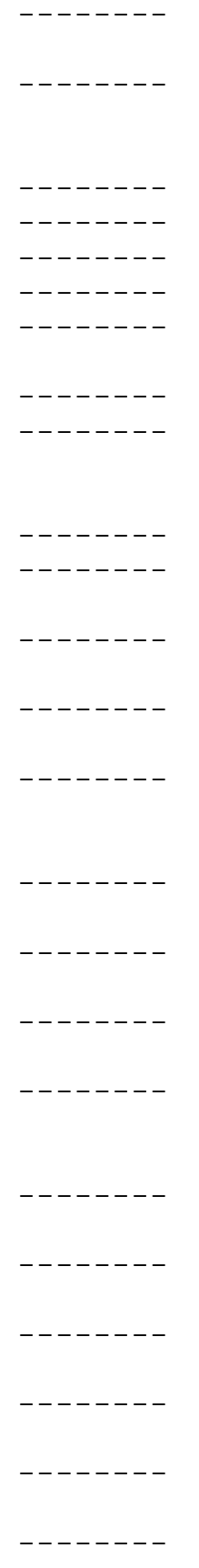
IANA Guidelines for IPv4 Multicast Address




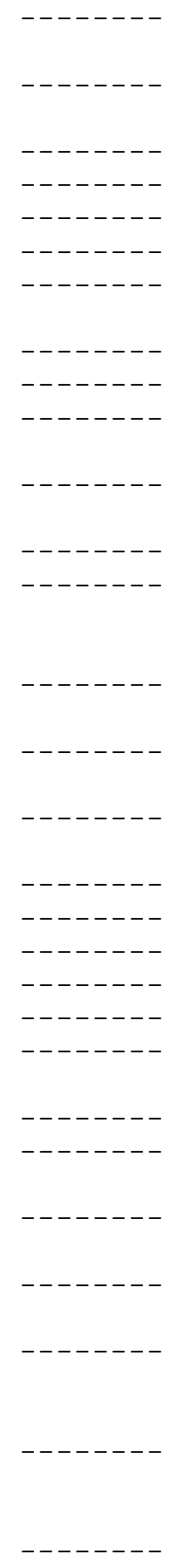




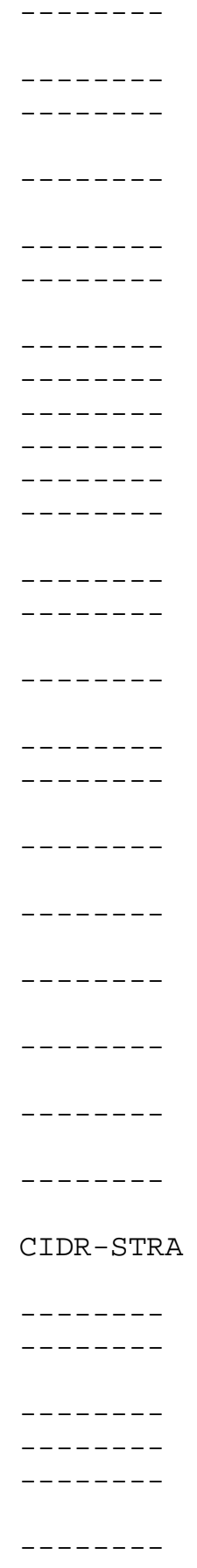

Structure of the IETF Administrative Support
Activity (IASA)

$4071 * 101$

BCP 101 Update for IPR Trust

IAB Processes for Management of IETF Liaison Relationships

Procedures for Handling Liaison Statements to and from the IETF

Terminology for Describing Internet Connectivity

$4371 * 101$

$4052 * 102$

$4053 * 103$

Embedding Globally-Routable Internet Addresses Considered Harmful

Randomness Requirements for Security

$4084 * 104$

Guidelines for Cryptographic Key Management

$4085 * 105$

IP Performance Metrics (IPPM) Metrics Registry

Deprecation of "ip6.int"

Tunneling Multiplexed Compressed RTP (TCRTP)

$4086 * 106$

$4107 * 107$

$4148 * 108$

$4159 * 109$

Guidelines for Authors and Reviewers of MIB

$4170 * 110$

Documents

RFC 4181 Update to Recognize the IETF Trust

$4181 * 111$

Prioritized Treatment of Specific OSPF Version 2

$4841 * 111$

Packets and Congestion Avoidance

The IETF Administrative Oversight Committee (IAOC)

Member Selection Guidelines and Process

BGP Communities for Data Collection 4384* 114

Guidelines and Registration Procedures for New URI 4395* 115 Schemes

IANA Allocations for Pseudowire Edge to Edge Emulation (PWE3)

Interworking between the Session Initiation

$4222 * 112$

$4333 * 113$

Protocol (SIP) and QSIG

Considerations for Lightweight Directory Access Protocol (LDAP) Extensions

Session Initiation Protocol (SIP) Call Control Conferencing for User Agents

Source-Specific Protocol Independent Multicast in $232 / 8$

Multicast Source Discovery Protocol (MSDP) Deployment Scenarios

Classless Inter-domain Routing (CIDR): The Internet Address Assignment and Aggregation Plan

Observed DNS Resolution Misbehavior

$4395 * 115$

$4446 * 116$

$4497 \star 117$

$4521 * 118$

$4579 * 119$

$4608 * 120$

$4611 * 121$

4632 * 122

Specifying Alternate Semantics for the Explicit Congestion Notification (ECN) Field

Procedures for Protocol Extensions and Variations Operation of Anycast Services

Network Address Translation (NAT) Behavioral

Requirements for Unicast UDP

Avoiding Equal Cost Multipath Treatment in MPLS Networks

$4697 * 123$

4774 * 124

$4775 * 125$

$4786 * 126$

$4787 * 127$

$4928 * 128$

RFC Editor

Informational

[Page 61] 


Change Process for Multiprotocol Label Switching
(MPLS) and Generalized MPLS (GMPLS) Protocols and
Procedures
IANA Considerations for OSPF
$----------\quad$ Symmetric RTP / RTP Control Protocol (RTCP)
$-------\quad$ Guidance for Authentication, Authorization, and
Accounting (AAA) Key Management
$------\quad$ Specifying New Congestion Control Algorithms
$-------\quad$ Email Submission Operations: Access and
Accountability Requirements
IP Multicast Requirements for a Network Address
Translator (NAT) and a Network Address Port
Translator (NAPT)

$4929 * 129$

$4940 * 130$

4961 * 131

$4962 * 132$

$5033 * 133$

$5068 * 134$

$5135 * 135$

3.6. Best Current Practice Ordered by RFC

[Note: an asterisk at the end of a line indicates a change from the previous edition of this document.]

\begin{tabular}{|c|c|c|c|}
\hline Mnemonic & Title & $\mathrm{BCP} \#$ & RFC\# \\
\hline-------- & $\begin{array}{l}\text { IP Multicast Requirements for a Network Address } \\
\text { Translator (NAT) and a Network Address Port } \\
\text { Translator (NAPT) }\end{array}$ & 135 & $5135 *$ \\
\hline----- & $\begin{array}{l}\text { Email Submission Operations: Access and } \\
\text { Accountability Requirements }\end{array}$ & 134 & 5068 * \\
\hline-------- & Specifying New Congestion Control Algorithms & 133 & $5033 *$ \\
\hline------ & $\begin{array}{l}\text { Guidance for Authentication, Authorization, and } \\
\text { Accounting (AAA) Key Management }\end{array}$ & 132 & 4962 * \\
\hline ------ー- & Symmetric RTP / RTP Control Protocol (RTCP) & 131 & 4961 * \\
\hline-------- & IANA Considerations for OSPF & 130 & 4940 * \\
\hline-------- & $\begin{array}{l}\text { Change Process for Multiprotocol Label Switching } \\
\text { (MPLS) and Generalized MPLS (GMPLS) Protocols } \\
\text { and Procedures }\end{array}$ & 129 & $4929 *$ \\
\hline-- & $\begin{array}{l}\text { Avoiding Equal Cost Multipath Treatment in MPLS } \\
\text { Networks }\end{array}$ & 128 & $4928 *$ \\
\hline-------- & $\begin{array}{l}\text { Handling Normative References to Standards-Track } \\
\text { Documents }\end{array}$ & 97 & $4897 *$ \\
\hline-------- & $\begin{array}{l}\text { Clarification of the Third Party Disclosure } \\
\text { Procedure in RFC } 3979\end{array}$ & 79 & $4879 *$ \\
\hline-------- & RFC 4181 Update to Recognize the IETF Trust & 111 & 4841 * \\
\hline-------- & $\begin{array}{l}\text { Network Address Translation (NAT) Behavioral } \\
\text { Requirements for Unicast UDP }\end{array}$ & 127 & $4787 \star$ \\
\hline-------- & Operation of Anycast Services & 126 & $4786 *$ \\
\hline-------- & Procedures for Protocol Extensions and Variations & 125 & $4775 *$ \\
\hline-------- & $\begin{array}{l}\text { Specifying Alternate Semantics for the Explicit } \\
\text { Congestion Notification (ECN) Field }\end{array}$ & 124 & 4774 * \\
\hline-------- & RFC 3978 Update to Recognize the IETF Trust & 78 & $4748 *$ \\
\hline
\end{tabular}




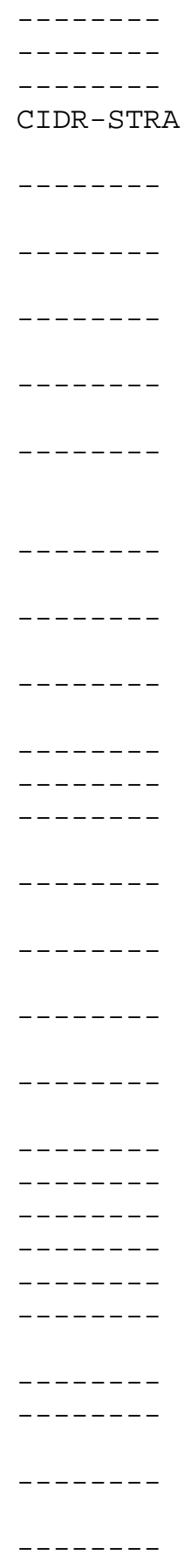

Observed DNS Resolution Misbehavior

$1234697 *$

Matching of Language Tags

$474647 *$

Tags for Identifying Languages

$474646 *$

Classless Inter-domain Routing (CIDR): The Internet Address Assignment and Aggregation Plan

Multicast Source Discovery Protocol (MSDP)

$1224632 *$ Deployment Scenarios

Source-Specific Protocol Independent Multicast in $232 / 8$

Session Initiation Protocol (SIP) Call Control Conferencing for User Agents

Considerations for Lightweight Directory Access Protocol (LDAP) Extensions

Internet Assigned Numbers Authority (IANA) Considerations for the Lightweight Directory Access Protocol (LDAP)

Interworking between the Session Initiation Protocol (SIP) and QSIG

IANA Allocations for Pseudowire Edge to Edge Emulation (PWE3)

Guidelines and Registration Procedures for New URI Schemes

BGP Communities for Data Collection

BCP 101 Update for IPR Trust

The IETF Administrative Oversight Committee

(IAOC) Member Selection Guidelines and Process

$1214611 *$

1204608 *

$1194579 *$

1184521 *

$644520 *$

Multipurpose Internet Mail Extensions (MIME)

Part Four: Registration Procedures

Media Type Specifications and Registration Procedures

Prioritized Treatment of Specific OSPF Version 2 Packets and Congestion Avoidance

Guidelines for Authors and Reviewers of MIB Documents

Tunneling Multiplexed Compressed RTP (TCRTP)

Deprecation of "ip6.int"

IP Performance Metrics (IPPM) Metrics Registry

Guidelines for Cryptographic Key Management

Randomness Requirements for Security

Embedding Globally-Routable Internet Addresses Considered Harmful

Terminology for Describing Internet Connectivity

Structure of the IETF Administrative Support Activity (IASA)

Procedures for Handling Liaison Statements to and from the IETF

IAB Processes for Management of IETF Liaison Relationships

$1174497 *$

$1164446^{*}$

$1154395 *$

$1144384 *$

$1014371^{*}$

$1134333^{*}$

$134289 *$

$134288 *$

$1124222 *$

$1114181 *$

1104170 *

109 4159*

$1084148 *$

107 4107*

$1064086 *$

$1054085 *$

1044084 *

$1014071 *$

103 4053*

1024052 *

[Page 63] 


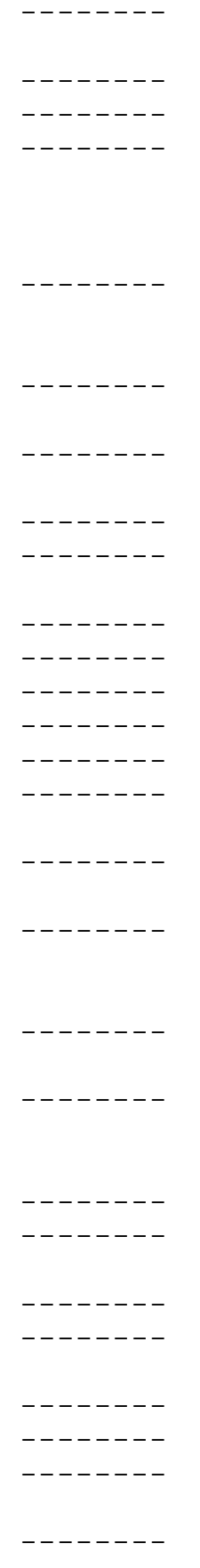

Early IANA Allocation of Standards Track Code

$1004020 *$ Points

Intellectual Property Rights in IETF Technology

$793979 *$

IETF Rights in Contributions

$783978 *$

The Internet Assigned Number Authority (IANA)

$993969 *$

Uniform Resource Identifier (URI) Parameter Registry for the Session Initiation Protocol (SIP)

The Internet Assigned Number Authority (IANA)

$983968 *$

Header Field Parameter Registry for the Session Initiation Protocol (SIP)

Clarifying when Standards Track Documents may Refer Normatively to Documents at a Lower Level

Procedures for Modifying the Resource reserVation Protocol (RSVP)

A Mission statement for the IETF

Updates to RFC 2418 Regarding the Management of IETF Mailing Lists

A Model for IETF Process Experiments

The IESG and RFC Editor Documents: Procedures

DNS IPv6 Transport Operational Guidelines

Registration Procedures for Message Header Fields

Advice for Internet Subnetwork Designers

IANA Considerations for the Point-to-Point Protocol (PPP)

Use of Interior Gateway Protocol (IGP) Metric as a second MPLS Traffic Engineering (TE) Metric

IAB and IESG Selection, Confirmation, and Recall Process: Operation of the Nominating and Recall Committees

Determining Strengths For Public Keys Used For Exchanging Symmetric Keys

Best Current Practices for Third Party Call Control (3pcc) in the Session Initiation Protocol (SIP)

Ingress Filtering for Multihomed Networks

Assigning Experimental and Testing Numbers Considered Useful

The IETF XML Registry

A Practice for Revoking Posting Rights to IETF Mailing Lists

Delegation of E.F.F.3.IP6.ARPA

IETF ISOC Board of Trustee Appointment Procedures

Session Initiation Protocol (SIP) Public Switched Telephone Network (PSTN) Call Flows

Session Initiation Protocol (SIP) Basic Call Flow Examples

$973967 *$

$963936 *$

$953935 *$

$943934 *$

93 3933*

92 3932*

$913901 *$

903864 *

893819

883818

$87 \quad 3785$

$10 \quad 3777$

863766

$85 \quad 3725$

$84 \quad 3704$

$82 \quad 3692$

813688

$83 \quad 3683$

$80 \quad 3681$

$77 \quad 3677$

763666

$75 \quad 3665$ 


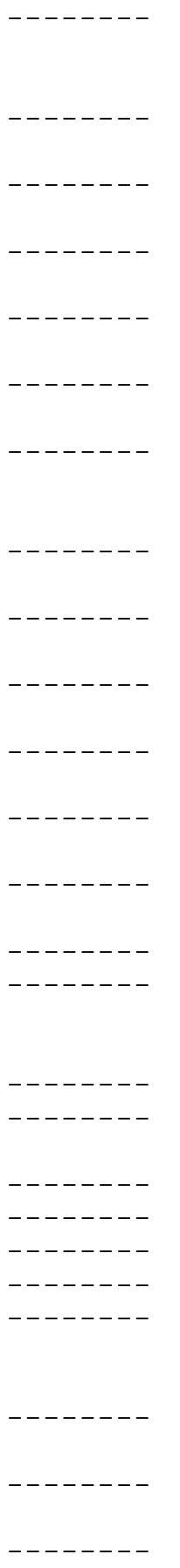

Coexistence between Version 1, Version 2, and

Version 3 of the Internet-standard Network

Management Framework

An IETF URN Sub-namespace for Registered

$73 \quad 3553$

Protocol Parameters

Guidelines for Writing RFC Text on Security Considerations

$72 \quad 3552$

TCP over Second (2.5G) and Third (3G) Generation 713481

Wireless Networks

Guidelines for the Use of Extensible Markup

$70 \quad 3470$

Language (XML) within IETF Protocols

TCP Performance Implications of Network Path

693449

Asymmetry

Layer Two Tunneling Protocol (L2TP) Internet Assigned Numbers Authority (IANA) Considerations Update

Change Process for the Session Initiation Protocol (SIP)

Uniform Resource Names (URN) Namespace Definition Mechanisms

Dynamic Delegation Discovery System (DDDS) Part 653405

Five: URI.ARPA Assignment Procedures

Session Initiation Protocol for Telephones $(\mathrm{SIP}-\mathrm{T})$ : Context and Architectures

Advice to link designers on link Automatic Repeat reQuest (ARQ)

Strong Security Requirements for Internet Engineering Task Force Standard Protocols

Inappropriate TCP Resets Considered Harmful

A Transient Prefix for Identifying Profiles under Development by the Working Groups of the Internet Engineering Task Force

Defining the IETF

IANA Considerations for IPv4 Internet Group Management Protocol (IGMP)

Guidelines for Evidence Collection and Archiving

$68 \quad 3438$

$67 \quad 3427$

663406

$63 \quad 3372$

623366

613365

603360

593349

On the use of HTTP as a substrate

IETF Guidelines for Conduct

GLOP Addressing in 233/8

Management Guidelines \& Operational Requirements for the Address and Routing Parameter Area Domain ("arpa")

IANA Guidelines for IPv4 Multicast Address Assignments

End-to-end Performance Implications of Links with Errors

End-to-end Performance Implications of Slow Links 483150 


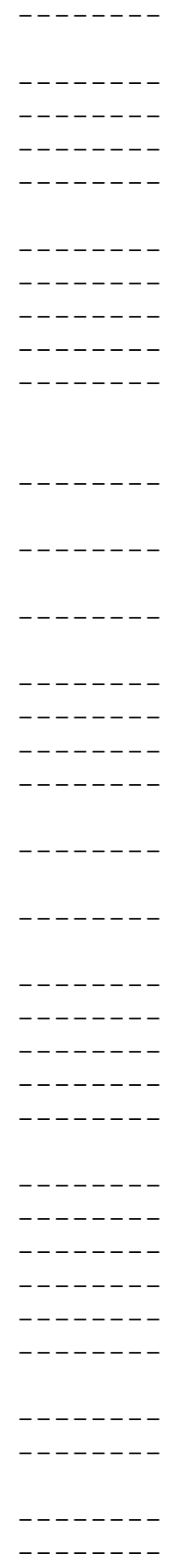

Recommended Internet Service Provider Security Services and Procedures IETF Discussion List Charter IANA Charset Registration Procedures

Use of HTTP State Management

Procedures and IANA Guidelines for Definition of New DHCP Options and Message Types

Domain Name System (DNS) IANA Considerations

Congestion Control Principles

Root Name Server Operational Requirements

Charter of the Internet Architecture Board (IAB)

Network Ingress Filtering: Defeating Denial of Service Attacks which employ IP Source Address Spoofing

IANA Allocation Guidelines For Values In the Internet Protocol and Related Headers

Guidelines for Writers of RTP Payload Format Specifications

Changing the Default for Directed Broadcasts in Routers

Reserved Top Level DNS Names

Media Feature Tag Registration Procedure

Anti-Spam Recommendations for SMTP MTAs

Enhancing TCP Over Satellite Channels using Standard Mechanisms

Advancement of MIB specifications on the IETF Standards Track

Guidelines for Writing an IANA Considerations section in RFCs

IETF Working Group Guidelines and Procedures

RSVP over ATM Implementation Guidelines

Administratively Scoped IP Multicast

Guide for Internet Standards Writers

Expectations for Computer Security Incident Response

Classless IN-ADDR.ARPA delegation

IETF Policy on Character sets and Languages

Use of DNS Aliases for Network Services

Selection and Operation of Secondary DNS Servers

Deployment of the Internet White Pages Service

Key words for use in RFCs to Indicate Requirement Levels

Internet Registry IP Allocation Guidelines

The Organizations Involved in the IETF Standards Process

The Internet Standards Process -- Revision 3

IRTF Research Group Guidelines and Procedures
463013

453005

192978

$44 \quad 2964$

432939

$42 \quad 2929$

$41 \quad 2914$

$40 \quad 2870$

392850

$38 \quad 2827$

$37 \quad 2780$

$36 \quad 2736$

$34 \quad 2644$

322606

312506

$30 \quad 2505$

$28 \quad 2488$

$27 \quad 2438$

$26 \quad 2434$

252418

$24 \quad 2379$

$23 \quad 2365$

$22 \quad 2360$

212350

$\begin{array}{ll}20 & 2317\end{array}$

$\begin{array}{ll}18 & 2277\end{array}$

$\begin{array}{ll}17 & 2219\end{array}$

$\begin{array}{ll}16 & 2182\end{array}$

$15 \quad 2148$

$14 \quad 2119$

$12 \quad 2050$

112028

92026

82014 


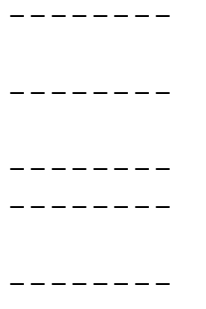

$$
\begin{array}{lc}
\text { Implications of Various Address Allocation } & 72008 \\
\text { Policies for Internet Routing } & 61930 \\
\text { Guidelines for creation, selection, and } & \\
\text { registration of an Autonomous System (AS) } & 51918 \\
\text { Address Allocation for Private Internets } & 41917 \\
\text { An Appeal to the Internet Community to Return } & 31915 \\
\text { Unused IP Networks (Prefixes) to the IANA } & \\
\text { Variance for The PPP Compression Control } & \\
\quad \text { Protocol and The PPP Encryption Control Protocol } &
\end{array}
$$

\begin{tabular}{|c|c|c|}
\hline Mnemonic & Title & RFC\# \\
\hline---- & $\begin{array}{l}\text { Experiment in Exploratory Group Formation within the } \\
\text { Internet Engineering Task Force (IETF) }\end{array}$ & $5111 *$ \\
\hline---- & $\begin{array}{l}\text { The Extensible Authentication Protocol-Internet Key } \\
\text { Exchange Protocol version } 2 \text { (EAP-IKEv2) Method }\end{array}$ & $5106 *$ \\
\hline-------- & $\begin{array}{l}\text { Using OpenPGP Keys for Transport Layer Security } \\
\text { (TLS) Authentication }\end{array}$ & $5081 *$ \\
\hline--- & Explicit Multicast (Xcast) Concepts and Options & $5058 *$ \\
\hline------- & Bundle Protocol Specification & $5050 *$ \\
\hline-------- & $\begin{array}{l}\text { IPv6 Router Advertisement Option for DNS } \\
\text { Configuration }\end{array}$ & $5006 *$ \\
\hline & Mobile IPv4 Fast Handovers & $4988 *$ \\
\hline-------- & $\begin{array}{l}\text { OSPF-xTE: Experimental Extension to OSPF for Traffic } \\
\text { Engineering }\end{array}$ & $4973 *$ \\
\hline-------- & DNS Security (DNSSEC) Opt-In & $4956 *$ \\
\hline-------- & Atom License Extension & $4946 *$ \\
\hline-------- & $\begin{array}{l}\text { Abstract Syntax Notation } X \text { (ASN.X) Representation of } \\
\text { Encoding Instructions for the XML Encoding Rules (XER }\end{array}$ & 4914 * \\
\hline------- & $\begin{array}{l}\text { Abstract Syntax Notation X (ASN.X) Representation of } \\
\text { Encoding Instructions for the Generic String } \\
\text { Encoding Rules (GSER) }\end{array}$ & $4913 *$ \\
\hline-------- & Abstract Syntax Notation X (ASN.X) & 4912 * \\
\hline-------- & $\begin{array}{l}\text { Encoding Instructions for the Robust XML Encoding } \\
\text { Rules (RXER) }\end{array}$ & 4911 * \\
\hline-------- & $\begin{array}{l}\text { Robust XML Encoding Rules (RXER) for Abstract Syntax } \\
\text { Notation One (ASN.1) }\end{array}$ & $4910 *$ \\
\hline-------- & $\begin{array}{l}\text { Multi-homing for small scale fixed network Using } \\
\text { Mobile IP and NEMO }\end{array}$ & $4908 *$ \\
\hline-------- & Low-Latency Handoffs in Mobile IPv4 & 4881 * \\
\hline-------- & Mobile IPv4 Regional Registration & $4857 *$ \\
\hline-------- & $\begin{array}{l}\text { An IPv6 Prefix for Overlay Routable Cryptographic } \\
\text { Hash Identifiers (ORCHID) }\end{array}$ & $4843 *$ \\
\hline
\end{tabular}

3.7. Experimental Protocols

[Note: an asterisk at the end of a line indicates a change from the previous edition of this document.] 


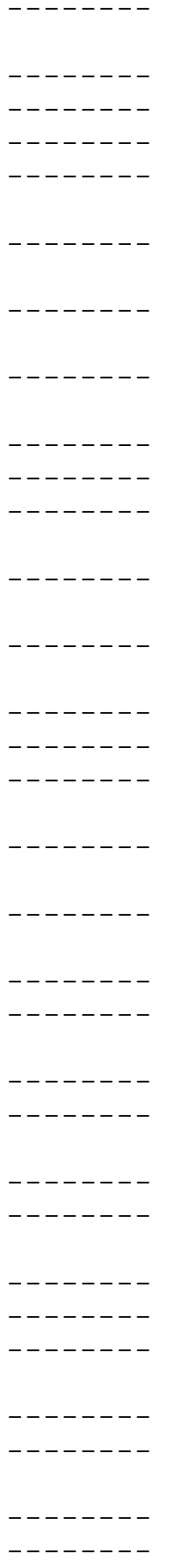

TCP Friendly Rate Control (TFRC): The Small-Packet

(SP) Variant

OSPF Link-Local Signaling

$4813 *$

Quick-Start for TCP and IP

$4782 *$

The Intrusion Detection Exchange Protocol (IDXP)

$4767 *$

The Intrusion Detection Message Exchange Format (IDMEF)

The EAP-PSK Protocol: A Pre-Shared Key Extensible

$4765 *$

Authentication Protocol (EAP) Method

Multiple Authentication Exchanges in the Internet Key Exchange (IKEv2) Protocol

The Dynamic Source Routing Protocol (DSR) for Mobile Ad Hoc Networks for IPv4

Netnews Administration System (NAS)

4764 *

IETF Operational Notes

TCP-Friendly Multicast Congestion Control (TFMCC): Protocol Specification

Improving the Robustness of TCP to Non-Congestion Events

Experiment in Long-Term Suspensions From Internet Engineering Task Force (IETF) Mailing Lists

Multicast Source Discovery Protocol (MSDP) MIB

IPv6 Node Information Queries

NEC's Simple Middlebox Configuration (SIMCO) Protocol Version 3.0

The Lightweight Directory Access Protocol (LDAP) Content Synchronization Operation

Lightweight Directory Access Protocol (LDAP) Turn Operation

The Managed Object Aggregation MIB

Repeated Authentication in Internet Key Exchange (IKEv2) Protocol

Derivation of DNS Name Predecessor and Successor

Web Distributed Authoring and Versioning (WebDAV) Redirect Reference Resources

Selectively Reliable Multicast Protocol (SRMP)

Sender Policy Framework (SPF) for Authorizing Use of Domains in E-Mail, Version 1

Purported Responsible Address in E-Mail Messages 4407*

Sender ID: Authenticating E-Mail

$4406 *$

SMTP Service Extension for Indicating the

$4405 *$

Responsible Submitter of an E-Mail Message

Neighbor Discovery Proxies (ND Proxy)

$4389 *$

Internet X.509 Public Key Infrastructure Repository

Locator Service

Calendar Access Protocol (CAP)

4324 *

Datatypes for Web Distributed Authoring and

Versioning (WebDAV) Properties 
ISATAP Intra-Site Automatic Tunnel Addressing Protocol (ISATAP)

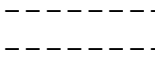

Hierarchical Mobile IPv6 Mobility Management (HMIPv6) and the Stream Control Transmission Protocol (SCTP)

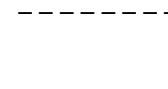

Russian Dolls Bandwidth Constraints Model for Diffserv-aware MPLS Traffic Engineering

Max Allocation with Reservation Bandwidth Constraints Model for Diffserv-aware MPLS Traffic Engineering \& Performance Comparisons

Maximum Allocation Bandwidth Constraints Model for Diffserv-aware MPLS Traffic Engineering

Fast Handovers for Mobile IPv6

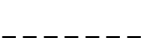

Context Transfer Protocol (CXTP)

Candidate Access Router Discovery (CARD)

$4126 *$

Instructions for Seamoby and Experimental Mobility Protocol IANA Allocations

BinaryTime: An Alternate Format for Representing Date and Time in ASN.1

Extensions to Support Efficient Carrying of Multicast Traffic in Layer-2 Tunneling Protocol (L2TP)

Maximum Transmission Unit Signalling Extensions for the Label Distribution Protocol

Protocol Independent Multicast - Dense Mode (PIM-DM) : Protocol Specification (Revised)

Real-time Transport Protocol (RTP) Payload Format for internet Low Bit Rate Codec (iLBC) Speech

Internet Low Bit Rate Codec (iLBC)

Negative-Acknowledgment (NACK)-Oriented Reliable Multicast (NORM) Building Blocks

Negative-acknowledgment (NACK)-Oriented Reliable Multicast (NORM) Protocol

Alternative Decision Making Processes for Consensus-Blocked Decisions in the IETF

4125 *

4068 * $4067 *$

$4066 *$

$4065 *$

$4049 *$

$4045 *$

3988 *

$3973 *$

$3952 *$

$3951 *$

$3941 *$

$3940 *$

3929*

FLUTE - File Delivery over Unidirectional Transport

Remote Service Discovery in the Service Location Protocol (SLP) via DNS SRV

Next Generation Structure of Management Information (SMIng) Mappings to the Simple Network Management Protocol (SNMP)

SMIng - Next Generation Structure of Management Information

Limited Slow-Start for TCP with Large Congestion Windows

Wave and Equation Based Rate Control (WEBRC) Building Block 


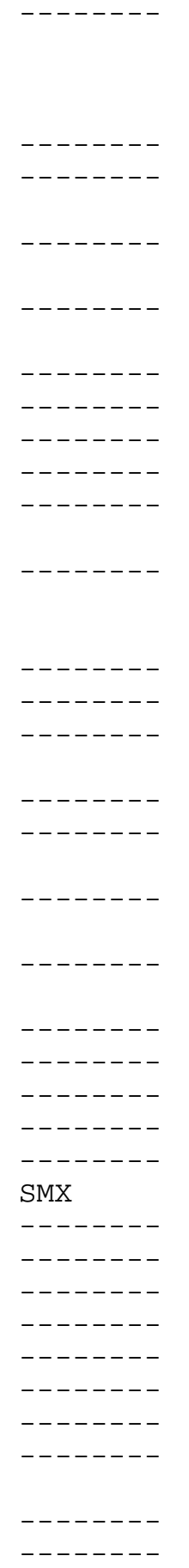

Using TCP Duplicate Selective Acknowledgement (DSACKs) and Stream Control Transmission Protocol (SCTP) Duplicate Transmission Sequence Numbers (TSNs) to Detect Spurious Retransmissions

Compact Forward Error Correction (FEC) Schemes Topology Dissemination Based on Reverse-Path Forwarding (TBRPF)

Domain Administrative Data in Lightweight Directory Access Protocol (LDAP)

The Mailbox Update (MUPDATE) Distributed Mailbox 3656 Database Protocol

Highspeed TCP for Large Congestion Windows

Optimized Link State Routing Protocol (OLSR)

Multicast Source Discovery Protocol (MSDP)

Ad hoc On-Demand Distance Vector (AODV) Routing

Robust Explicit Congestion Notification (ECN) Signaling with Nonces

Using Extensible Markup Language-Remote Procedure Calling (XML-RPC) in Blocks Extensible Exchange Protocol (BEEP)

Mesh-enhanced Service Location Protocol (mSLP)

The Eifel Detection Algorithm for TCP

TCP Congestion Control with Appropriate Byte Counting $(\mathrm{ABC})$

Layered Coding Transport (LCT) Building Block

Asynchronous Layered Coding (ALC) Protocol Instantiation

Simple Network Management Protocol Over Transmission Control Protocol Transport Mapping

Select and Sort Extensions for the Service Location Protocol (SLP)

The Application Exchange (APEX) Presence Service 3343

Dual Stack Hosts Using "Bump-in-the-API" (BIA) 3338

Policy-Based Accounting

PGM Reliable Transport Protocol Specification 3208

Domain Security Services using S/MIME 3183

Script MIB Extensibility Protocol Version 1.1 3179

ISO/IEC 9798-3 Authentication SASL Mechanism 3163

Electronic Signature Policies

3125

A DNS RR Type for Lists of Address Prefixes (APL RR) 3123

Finding an RSIP Server with SLP 3105

RSIP Support for End-to-end IPsec 3104

Realm Specific IP: Protocol Specification 3103

Realm Specific IP: Framework 3102

OpenLDAP Root Service An experimental LDAP referral 3088 service

Notification and Subscription for SLP 3082

MPLS Loop Prevention Mechanism 3063

RFC Editor

Informational

[Page 70] 


\begin{tabular}{|c|c|c|}
\hline & $\begin{array}{l}\text { nternet X.509 Public Key Infrastructure Data } \\
\text { Validation and Certification Server Protocols }\end{array}$ & \\
\hline-------- & Unified Memory Space Protocol Specification & 3018 \\
\hline SAP & Session Announcement Protocol & \\
\hline-------- & Protocol Independent Multicast MIB for IPv4 & \\
\hline MASC & The Multicast Address-Set Claim (MASC) Protocol & \\
\hline-------- & Generic AAA Architecture & \\
\hline-------- & $\begin{array}{l}\text { DNS Extensions to Support IPv6 Address Aggregation } \\
\text { and Renumbering }\end{array}$ & \\
\hline-------- & TCP Congestion Window Validation & 61 \\
\hline TSWTCM & A Time Sliding Window Three Colour Marker (TSWTCM) & \\
\hline-------- & OSPF over ATM and Proxy-PAR & \\
\hline $\mathrm{PPP}-\mathrm{SDL}$ & $\begin{array}{l}\text { PPP over Simple Data Link (SDL) using SONET/SDH with } \\
\text { ATM-like framing }\end{array}$ & \\
\hline-------- & $\begin{array}{l}\text { Diffie-Helman USM Key Management Information Base } \\
\text { and Textual Convention }\end{array}$ & \\
\hline-------- & An HTTP Extension Framework & 2774 \\
\hline-------- & Encryption using KEA and SKIPJACK & \\
\hline-------- & Sampling of the Group Membership in RTP & \\
\hline-------- & $\begin{array}{l}\text { Definitions of Managed Objects for Service Level } \\
\text { Agreements Performance Monitoring }\end{array}$ & \\
\hline HTCP & Hyper Text Caching Protocol (HTCP/0.0) & 275 \\
\hline-------- & RTFM: New Attributes for Traffic Flow Measurement & \\
\hline-------- & PPP EAP TLS Authentication Protocol & \\
\hline-------- & SPKI Certificate Theory & \\
\hline-------- & SPKI Requirements & 26 \\
\hline-------- & QoS Routing Mechanisms and OSPF Extensions & 26 \\
\hline DNS & Binary Labels in the Domain Name System & \\
\hline-------- & The Secure HyperText Transfer Protocol & \\
\hline-------- & Security Extensions For HTML & \\
\hline-------- & LDAPv2 Client vs. the Index Mesh & 26 \\
\hline-------- & Registration Procedures for SOIF Template Types & 26 \\
\hline-------- & CIP Index Object Format for SOIF Objects & 26 \\
\hline-------- & $\begin{array}{l}\text { A Tagged Index Object for use in the Common Indexing } \\
\text { Protocol }\end{array}$ & \\
\hline-------- & $\begin{array}{l}\text { An LDAP Control and Schema for Holding Operation } \\
\text { Signatures }\end{array}$ & 2649 \\
\hline-------- & Mapping between LPD and IPP Protocols & 2569 \\
\hline IPP-RAT & $\begin{array}{l}\text { Rationale for the Structure of the Model and } \\
\text { Protocol for the Internet Printing Protocol }\end{array}$ & 256 \\
\hline IPP-DG & Design Goals for an Internet Printing Protocol & 256 \\
\hline DNS-INFO & Detached Domain Name System (DNS) Information & 254 \\
\hline PHOTURIS-E & Photuris: Extended Schemes and Attributes & 252 \\
\hline PHOTURIS-S & Photuris: Session-Key Management Protocol & 252 \\
\hline ICMP - SEC & ICMP Security Failures Messages & 252 \\
\hline NHRP-MNHCS & NHRP with Mobile NHCs & 252 \\
\hline-------- & URI Resolution Services Necessary for URN Resolution & \\
\hline MARS-SCSP & A Distributed MARS Service Using SCSP & \\
\hline
\end{tabular}




\begin{tabular}{|c|c|c|}
\hline-------- & Domain Names and Company Name Retrieval & 15 \\
\hline RTP-MPEG & RTP Payload Format for Bundled MPEG & 2343 \\
\hline-------- & $\begin{array}{l}\text { Intra-LIS IP multicast among routers over ATM using } \\
\text { Sparse Mode PIM }\end{array}$ & 2337 \\
\hline-------- & The Safe Response Header Field & \\
\hline LDAP-NIS & $\begin{array}{l}\text { An Approach for Using LDAP as a Network Information } \\
\text { Service }\end{array}$ & \\
\hline HTTP-RVSA & HTTP Remote Variant Selection Algorithm -- RVSA/1.0 & \\
\hline TCN-HTTP & Transparent Content Negotiation in HTTP & \\
\hline TOP T-COMPO & Telnet Com Port Control Option & \\
\hline-------- & A Trivial Convention for using HTTP in URN Resolution & \\
\hline MAP-MAIL & $\begin{array}{l}\text { MaXIM-11 - Mapping between X.400 / Internet mail and } \\
\text { Mail-11 mail }\end{array}$ & \\
\hline MIME-ODA & A MIME Body Part for ODA & \\
\hline OSPF-DIG & OSPF with Digital Signatures & \\
\hline IP-SCSI & $\begin{array}{l}\text { Encapsulating IP with the Small Computer system } \\
\text { Interface }\end{array}$ & \\
\hline $\mathrm{X} .500-\mathrm{NAME}$ & Managing the X.500 Root Naming Context & \\
\hline GKMP - ARCH & Group Key Management Protocol (GKMP) Architecture & \\
\hline GKMP-SPEC & Group Key Management Protocol (GKMP) Specification & \\
\hline TFTP-MULTI & TFTP Multicast Option & \\
\hline IP-Echo & IP Echo Host Service & \\
\hline TOP T-CHARS & TELNET CHARSET Option & \\
\hline URAS & Uniform Resource Agents (URAs) & \\
\hline GPS-AR & GPS-Based Addressing and Routing & \\
\hline ETETP & $\begin{array}{l}\text { Experiments with a Simple File Transfer Protocol for } \\
\text { Radio Links using Enhanced Trivial File Transfer } \\
\text { Protocol (ETFTP) }\end{array}$ & \\
\hline SMKD & Scalable Multicast Key Distribution & \\
\hline DNS-LOC & $\begin{array}{l}\text { A Means for Expressing Location Information in the } \\
\text { Domain Name System }\end{array}$ & \\
\hline SGML-MT & SGML Media Types & \\
\hline CONT-MT & Message/External-Body Content-ID Access Type & \\
\hline UNARP & ARP Extension - UNARP & \\
\hline ESP 3DES & The ESP Triple DES Transform & \\
\hline-------- & SMTP 521 Reply Code & \\
\hline-------- & SMTP Service Extension for Checkpoint/Restart & \\
\hline-------- & $\begin{array}{l}\text { Communicating Presentation Information in Internet } \\
\text { Messages: The Content-Disposition Header }\end{array}$ & \\
\hline-------- & Schema Publishing in X.500 Directory & 1804 \\
\hline-------- & MHS use of the X.500 Directory to support MHS Routing & \\
\hline-------- & Class A Subnet Experiment & 1797 \\
\hline TCP / IPXMIB & TCP/IPX Connection Mib Specification & 179 \\
\hline-------- & TCP And UDP Over IPX Networks With Fixed Path MTU & \\
\hline ICMP-DM & ICMP Domain Name Messages & 1788 \\
\hline CLNP-MULT & Host Group Extensions for CLNP Multicasting & \\
\hline OSPF-OVFL & OSPF Database Overflow & 176 \\
\hline RWP & Remote Write Protocol - Version 1.0 & \\
\hline
\end{tabular}




\begin{tabular}{|c|c|c|}
\hline NARP & NBMA Address Resolution Protocol (NARP) & 1735 \\
\hline DNS-ENCODE & DNS Encoding of Geographical Location & 1712 \\
\hline $\mathrm{TCP}-\mathrm{POS}$ & An Extension to TCP : Partial Order Service & 1693 \\
\hline $\mathrm{T} / \mathrm{TCP}$ & $\begin{array}{l}\text { T/TCP -- TCP Extensions for Transactions Functional } \\
\text { Specification }\end{array}$ & 1644 \\
\hline MIME-UNI & Using Unicode with MIME & 1641 \\
\hline FOOBAR & FTP Operation Over Big Address Records & \\
\hline X500-CHART & Charting Networks in the $\mathrm{x} .500$ Directory & 16 \\
\hline X500-DIR & Representing IP Information in the X.500 Directory & 1608 \\
\hline SNMP-DP I & $\begin{array}{l}\text { Simple Network Management Protocol Distributed } \\
\text { Protocol Interface Version } 2.0\end{array}$ & 159 \\
\hline CLNP-TUBA & Use of ISO CLNP in TUBA Environments & 1561 \\
\hline REM-PRINT & $\begin{array}{l}\text { Principles of Operation for the TPC.INT Subdomain: } \\
\text { Remote Printing -- Technical Procedures }\end{array}$ & 1528 \\
\hline DASS & DASS - Distributed Authentication Security Service & 1507 \\
\hline EHF-MAIL & Encoding Header Field for Internet Messages & 1505 \\
\hline RAP & RAP: Internet Route Access Protocol & 1476 \\
\hline $\mathrm{TP}-\mathrm{IX}$ & TP/IX: The Next Internet & 1475 \\
\hline-------- & $\begin{array}{l}\text { Routing Coordination for X.400 MHS Services Within a } \\
\text { Multi Protocol / Multi Network Environment Table } \\
\text { Format V3 for Static Routing }\end{array}$ & 1465 \\
\hline-------- & $\begin{array}{l}\text { Using the Domain Name system To store Arbitrary } \\
\text { String Attributes }\end{array}$ & 1464 \\
\hline IRCP & Internet Relay Chat Protocol & 1459 \\
\hline SIFT & SIFT/UFT: Sender-Initiated/Unsolicited File Transfer & 1440 \\
\hline DIR-ARP & Directed ARP & 1433 \\
\hline TEL-SPX & Telnet Authentication: SPX & 1412 \\
\hline TEL-KER & Telnet Authentication: Kerberos Version 4 & 1411 \\
\hline TRACE-IP & Traceroute Using an IP Option & 1393 \\
\hline DNS-IP & An Experiment in DNS Based IP Routing & 1383 \\
\hline $\mathrm{RMCP}$ & Remote Mail Checking Protocol & 1339 \\
\hline MSP 2 & Message Send Protocol 2 & 1312 \\
\hline DSLCP & Dynamically Switched Link Control Protocol & 1307 \\
\hline-------- & $\mathrm{X} .500$ and Domains & 1279 \\
\hline IN-ENCAP & $\begin{array}{l}\text { Scheme for an internet encapsulation protocol: } \\
\text { Version } 1\end{array}$ & 1241 \\
\hline CLNS-MIB & $\begin{array}{l}\text { CLNS MIB for use with Connectionless Network } \\
\text { Protocol (ISO 8473) and End System to Intermediate } \\
\text { System (ISO 9542) }\end{array}$ & 1238 \\
\hline CFDP & Coherent File Distribution Protocol & 1235 \\
\hline IP-AX. 25 & Internet protocol encapsulation of AX.25 frames & 1226 \\
\hline ALERTS & $\begin{array}{l}\text { Techniques for managing asynchronously generated } \\
\text { alerts }\end{array}$ & 1224 \\
\hline MPP & Message Posting Protocol (MPP) & 1204 \\
\hline SNMP-BULK & Bulk Table Retrieval with the SNMP & 1187 \\
\hline DNS-RR & New DNS RR Definitions & 1183 \\
\hline IMAP 2 & Interactive Mail Access Protocol: Version 2 & 1176 \\
\hline
\end{tabular}




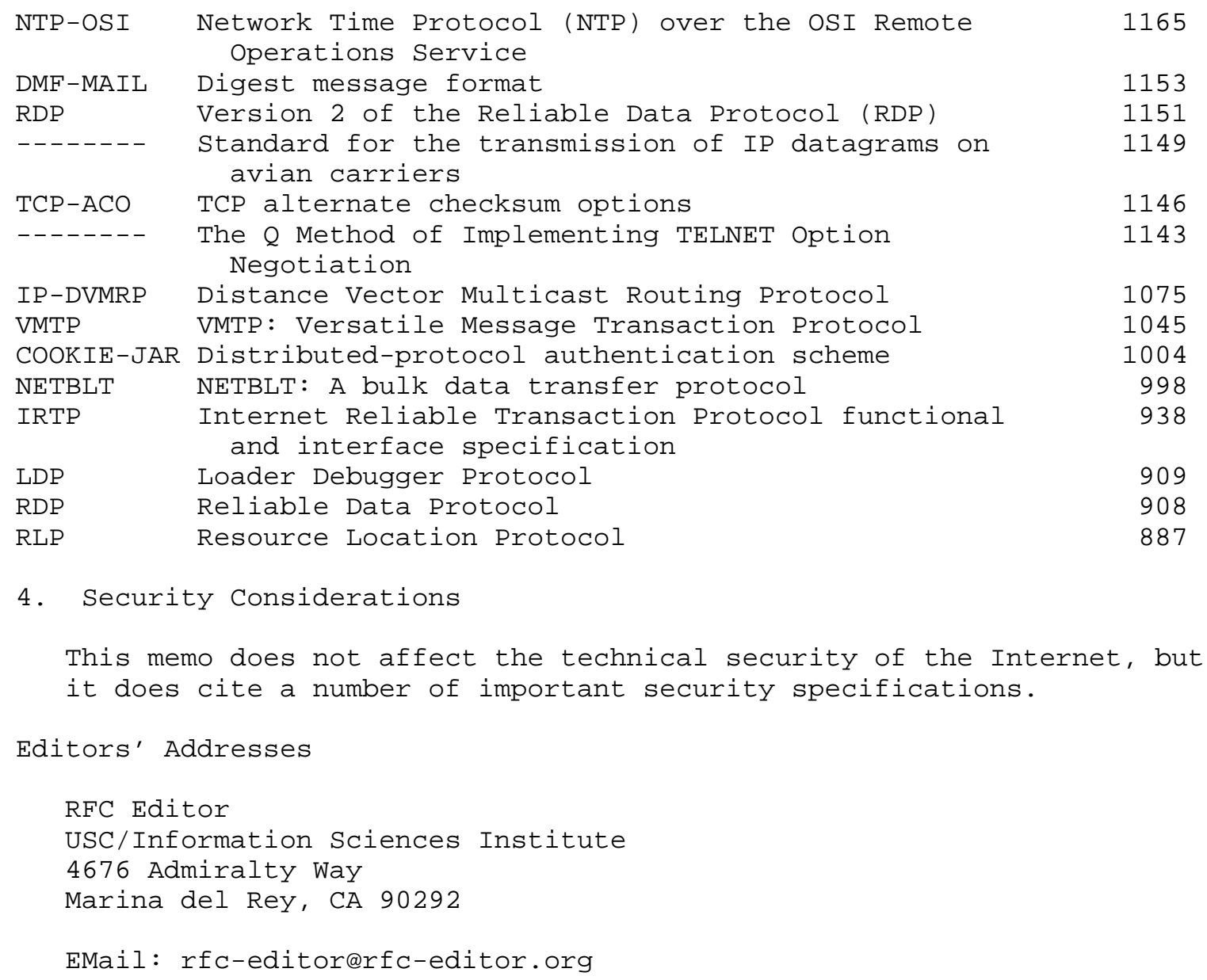


Full Copyright statement

Copyright (C) The IETF Trust (2008).

This document is subject to the rights, licenses and restrictions contained in BCP 78 and at http://www.rfc-editor.org/copyright.html, and except as set forth therein, the authors retain all their rights.

This document and the information contained herein are provided on an "AS IS" basis and THE CONTRIBUTOR, THE ORGANIZATION HE/SHE REPRESENTS OR IS SPONSORED BY (IF ANY), THE INTERNET SOCIETY, THE IETF TRUST AND THE INTERNET ENGINEERING TASK FORCE DISCLAIM ALL WARRANTIES, EXPRESS OR IMPLIED, INCLUDING BUT NOT LIMITED TO ANY WARRANTY THAT THE USE OF THE INFORMATION HEREIN WILL NOT INFRINGE ANY RIGHTS OR ANY IMPLIED WARRANTIES OF MERCHANTABILITY OR FITNESS FOR A PARTICULAR PURPOSE.

Intellectual Property

The IETF takes no position regarding the validity or scope of any Intellectual Property Rights or other rights that might be claimed to pertain to the implementation or use of the technology described in this document or the extent to which any license under such rights might or might not be available; nor does it represent that it has made any independent effort to identify any such rights. Information on the procedures with respect to rights in RFC documents can be found in BCP 78 and BCP 79 .

Copies of IPR disclosures made to the IETF Secretariat and any assurances of licenses to be made available, or the result of an attempt made to obtain a general license or permission for the use of such proprietary rights by implementers or users of this specification can be obtained from the IETF on-line IPR repository at http://www.ietf.org/ipr.

The IETF invites any interested party to bring to its attention any copyrights, patents or patent applications, or other proprietary rights that may cover technology that may be required to implement this standard. Please address the information to the IETF at ietf-ipreietf.org. 